\title{
Serious Game Engineering and Lighting Models for the Realistic Emulation of 5G Systems
}

\author{
Departamento de Comunicaciones \\ Universitat Politècnica de València
}

\author{
A thesis submitted for the degree of \\ Doctor por la Universitat Politècnica de València \\ Valencia, November 2019
}

Author:

Saúl Adrián Inca Sánchez

Supervisors:

Dr. Jose F. Monserrat del Río Dr. David Martín-Sacristán Gandía 

To my parents, to my brother and my sister, for all their support along this journey. 



\section{Abstract}

The fifth generation of mobile communications, $5 \mathrm{G}$, promises to be a technological revolution that goes beyond multiplying the data transmission speed of its predecessors. It aims at supporting a large number of devices and reach latencies very close to 1 millisecond. In order to meet these ambitious requirements, new enabling technologies have been researched. One of these is the use of millimetre-wave bands $(\mathrm{mmW})$, in which a large amount of spectrum is available.

Complex channel models are required to predict radio channel characteristics and reliably evaluate $5 \mathrm{G}$ performance in the $\mathrm{mmW}$ bands. Specifically, the most accurate propagation models are those based on ray tracing, but their high computational cost makes them unfeasible for radio channel characterization in complex scenarios. On the other hand, in recent years, the video game sector has developed powerful tools to model the propagation of light in super realistic scenarios. Given the spectral closeness between the visible spectrum and the mmW waves, the present Thesis has studied the application of light propagation modeling tools from game engines for radio channel modeling in mmW.

This Thesis proposes a model for estimating propagation losses in mmW called "Light Intensity Model" (LIM). Using this model, based on the lighting processes performed by the game engines, the signal transmitters are replaced by light sources and the light intensity received at a point is translated into signal strength in mmW through a simple polynomial function.

One of the advantages of using the game engines is their great capacity and the ease with which the user can create super realistic scenarios that faithfully represent the geometry of scenarios where the radio channel is to be evaluated. In this way, accurate estimates of propagation losses can be obtained. The estimation of propagation losses with LIM has been compared with measurement campaigns in the $28 \mathrm{GHz}$ and $73 \mathrm{GHz}$ bands and with other propagation models. As a result, the LIM estimation error is smaller than the current stochastic models and is comparable with the ray tracing model. In addition, the computational cost of LIM compared to ray tracing is 130 times lower, allowing the use of LIM in highly complex scenarios for real-time radio channel estimation.

The game engines allow characterizing in a different way the interaction of the materials with the light configuring the normal map of their surfaces and their scattering and reflection functions. In this Thesis, it has been determined the characterization of several materials that best fits to laboratory measure- 
ments made in a controlled scenario in the $28 \mathrm{GHz}$ band. The LIM model using materials with this optimal characterization reduces by more than $50 \%$ its estimation error with respect to the application of LIM with default materials, while its computational cost remains 26 times lower than conventional ray tracing models.

Finally, a first version of a platform for the emulation of $5 \mathrm{G}$ systems has been developed on a game engine, which is the starting point for a complete $5 \mathrm{G}$ emulator. This platform not only contains the LIM model but also includes several 5G use cases in super realistic environments. The platform, which is based on the concept of "Serious Game Engineering", breaks the limitations of mobile network simulators in terms of visualization capabilities and user interaction with network components in real time. 


\section{Resumen}

La quinta generación de comunicaciones móviles, 5G, promete ser una revolución tecnológica que vaya más allá de multiplicar la velocidad de transmisión de datos de sus predecesoras. Pretende soportar una gran cantidad de dispositivos y alcanzar latencias muy cercanas a 1 milisegundo. Para satisfacer estos ambiciosos requisitos, se han investigado nuevas tecnologías habilitadoras. Una de ellas es el uso de las bandas de ondas milimétricas ( $\mathrm{mmW}$ ) en las cuales hay una gran cantidad de espectro disponible.

Para predecir las características del canal radio y evaluar las prestaciones de la $5 \mathrm{G}$ de forma fiable en las bandas $\mathrm{mmW}$ se requieren modelos de canal complejos. Concretamente, los modelos de propagación más precisos son los basados en trazado de rayos, pero su alto costo computacional los hacen inviables para la caracterización del canal radio en escenarios complejos. Por otro lado, en los últimos años, la tecnología de videojuegos ha desarrollado potentes herramientas para modelar la propagación de la luz en escenarios superrealistas. Dada la cercanía espectral entre el espectro visible y las ondas mmW, la presente Tesis ha estudiado la aplicación de las herramientas de modelado de propagación de la luz de los motores de juego para el modelado del canal radio en $\mathrm{mmW}$.

Esta Tesis propone un modelo de estimación de las pérdidas de propagación en mmW llamado "Modelo de Intensidad de Luz" (LIM). Usando este modelo, basado en los procesos de iluminación realizados por los motores de juego, los transmisores de señal se sustituyen por focos de luz y la intensidad lumínica recibida en un punto se traduce a potencia de señal en milimétricas a través de una función polinómica sencilla.

Una de las ventajas de usar los motores de juego es su gran capacidad y la facilidad que tiene el usuario para crear escenarios superrealistas que representen fielmente la geometría de escenarios donde se quiera evaluar el canal radio. De esta forma se pueden obtener estimaciones precisas de las pérdidas de propagación. La estimación de las pérdidas de propagación con LIM ha sido comparada con campañas de medida en las bandas de $28 \mathrm{GHz}$ y $73 \mathrm{GHz}$ y con otros modelos de propagación. Como resultado, el error de estimación de LIM es menor que los modelos estocásticos actuales y es comparable con el modelo de trazado de rayos. Y, además, el coste computacional de LIM comparado con el trazado de rayos es 130 veces menor, lo que posibilita el uso de LIM en escenarios altamente complejos para la estimación del canal radio en tiempo real. 
Los motores de juego permiten caracterizar de forma diferente la interacción de los materiales con la luz configurando el mapa de normales de sus superficies y sus funciones de dispersión y reflexión. En esta Tesis se ha determinado la caracterización de varios materiales que mejor se ajusta a medidas de laboratorio realizadas en un escenario controlado en la banda de $28 \mathrm{GHz}$. El modelo de LIM empleando materiales con esta caracterización óptima reduce más de un $50 \%$ su error de estimación con respecto a la aplicación de LIM con los materiales por defecto, mientras que su coste computacional sigue siendo 26 veces menor que el modelo de trazado de rayos.

Finalmente, se ha desarrollado sobre un motor de juego una primera versión de plataforma para la emulación de los sistemas $5 \mathrm{G}$ que es el punto de partida para un emulador completo de 5G. Esta plataforma no sólo contiene el modelo de LIM sino que incluye varios casos de uso de la $5 \mathrm{G}$ en entornos superrealistas. La plataforma, que se basa en el concepto de "Serious Game Engineering", rompe las limitaciones de los simuladores de redes móviles en cuanto a las capacidades de visualización e interacción del usuario con los componentes de la red en tiempo real. 


\section{Resum}

La cinquena generació de comunicacions mòbils, 5G, promet ser una revolució tecnològica que vaja més enllà de multiplicar la velocitat de transmissió de dades de les seues predecessores. Pretén suportar una gran quantitat de dispositius i aconseguir latències molt pròximes a 1 mil-lisegon. Per a satisfer aquests ambiciosos requisits, s'han investigat noves tecnologies habilitadores. Una d'elles és l'ús de les bandes d'ones mil-limètriques ( $\mathrm{mmW}$ ) en les quals hi ha una gran quantitat d'espectre disponible.

Per a predir les característiques del canal ràdio i avaluar les prestacions de la $5 \mathrm{G}$ de forma fiable en les bandes $\mathrm{mmW}$ es requereixen models de canal complexos. Concretament, els models de propagació més precisos són els basats en traçat de rajos, però el seu alt cost computacional els fan inviables per a la caracterització del canal ràdio en escenaris complexos. D'altra banda, en els últims anys, la tecnologia de videojocs ha desenvolupat potents eines per a modelar la propagació de la llum en escenaris superrealistes. Donada la proximitat espectral entre l'espectre visible i les ones $\mathrm{mmW}$, la present Tesi ha estudiat l'aplicació de les eines de modelatge de propagació de la llum dels motors de joc per al modelatge del canal radie en mmW.

Aquesta Tesi proposa un model d'estimació de les pèrdues de propagació en mmW anomenat "Model d'Intensitat de Llum" (LIM). Usant aquest model, basat en els processos d'il-luminació realitzats pels motors de joc, els transmissors de senyal se substitueixen per focus de llum i la intensitat lumínica rebuda en un punt es tradueix a potència de senyal en mil-limètriques a través d'una funció polinòmica senzilla.

Una dels avantatges d'usar els motors de joc és la seua gran capacitat i la facilitat que té l'usuari per a crear escenaris superrealistes que representen fidelment la geometria d'escenaris on es vulga avaluar el canal ràdio. D'aquesta forma es poden obtindre estimacions precises de les pèrdues de propagació. L'estimació de les pèrdues de propagació amb LIM ha sigut comparada amb campanyes de mesura en les bandes de $28 \mathrm{GHz}$ i $73 \mathrm{GHz}$ i amb altres models de propagació. Com a resultat, l'error d'estimació de LIM és menor que els models estocàstics actuals i és comparable amb el model de traçat de rajos. I, a més, el cost computacional de LIM comparat amb el traçat de rajos és 130 vegades menor, la qual cosa possibilita l'ús de LIM en escenaris altament complexos per a l'estimació del canal ràdio en temps real.

Els motors de joc permeten caracteritzar de forma diferent la interacció dels materials amb la llum configurant el mapa de normals de les seues superfícies 
i les seues funcions de dispersió i reflexió. En aquesta Tesi s'ha determinat la caracterització de diversos materials que s'ajusta millor a mesures de laboratori realitzades en un escenari controlat en la banda de $28 \mathrm{GHz}$. El model de LIM emprant materials amb aquesta caracterització òptima redueix més d'un $50 \%$ el seu error d'estimació respecte a l'aplicació de LIM amb els materials per defecte, mentre que el seu cost computacional continua sent 26 vegades menor que el model de traçat de rajos.

Finalment, s'ha desenvolupat sobre un motor de joc una primera versió de plataforma per a l'emulació dels sistemes $5 \mathrm{G}$ que és el punt de partida per a un emulador complet de 5G. Aquesta plataforma no solament conté el model de LIM sinó que inclou diversos casos d'ús de la 5G en entorns superrealistes. La plataforma, que es basa en el concepte de "Serious Game Engineering", trenca les limitacions dels simuladors de xarxes mòbils quant a les capacitats de visualització i interacció de l'usuari amb els components de la xarxa en temps real. 


\section{Acknowledgements}

First of all, my deepest and most sincere thanks to my supervisors. I thank Prof. Jose F. Monserrat for guiding me and giving me the support along this journey that began when I started the MSc. in 2013 until now. A very special thanks to Dr. David Martín-Sacristán, who has helped me and given me the support for the development of this Thesis and has shown me his great quality as a professional and as a human.

In the same way, a special thanks to all my ex-colleagues of the National Institute of Telecommunications Research and Training INICTEL-UNI of Peru and to my colleagues of the research group of mobile communications of the iTEAM of the UPV, for giving me all their support to reach this objective. I would also like to thank Prof. Andrés Alayón for giving me the opportunity to stay in his research group at the University of Twente, which has enriched my scientific training and will be an unforgettable experience.

I thank Dr. Vittorio Degli-Esposti, Dr. Paolo Grazioso, and Dr. José María Molina for their work as reviewers of the Thesis. Special thanks also to Dr. Alberto González, Dr. Carmen Botella and Dr. Paolo Grazioso for acting as members of the committee.

Finally, I would like to express my deepest gratitude to those closest to me. First to my parents, David and Yolanda, that nothing of this would have been possible without their help and a lifetime wouldn't be enough to thank them for all they've done for me. Second, to my brother and my sister, who together with my parents are the most important people in my life. Finally, to all my family and friends around the world for helping and encouraging me.

Thank you all very much. 



\section{Table of contents}

Acronyms $\quad$ v

Notation $\quad$ ix

1 Introduction 1

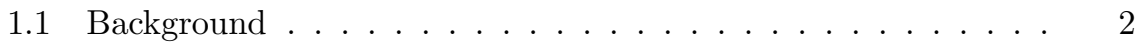

1.1.1 Towards Fifth Generation $(5 \mathrm{G}) \ldots . . . . . .22$

1.1 .2 5G Requirements . . . . . . . . . . . . . . . . . . 3

1.1 .3 Use Cases . . . . . . . . . . . . . . . . . . . . . . . . 4

$1.1 .45 \mathrm{G}$ Enablers . . . . . . . . . . . . . . . . . . 6

1.1.5 5G Spectrum Requirements ........... 8

1.1.6 5G Channel Model Requirements and Propagation Scenarios ................... . . . 10

1.2 State of the Art Analysis . . . . . . . . . . . . . . . 11

1.2.1 5G Radio Propagation Channel . . . . . . . . . . . 11

1.2.2 Propagation Channel models above $6 \mathrm{GHz}$. . . . . . . 15

1.2.3 Serious Game Engineering . . . . . . . . . . . . . . . 18

1.3 Problem and Thesis Scope . . . . . . . . . . . . . . 21

1.4 Thesis Objectives . . . . . . . . . . . . . . . . . . . . . . 22

1.5 Thesis Outline . . . . . . . . . . . . . . . . . . 23

1.6 Thesis Publications ................... 24

2 Evaluation of Path Loss Channel Models for 5G 25

2.1 Introduction . . . . . . . . . . . . . . . . . 26

2.2 IMT-2020 and 3GPP Large-Scale Channel Model . . . . . . . . 26

2.2.1 Large-Scale Model . . . . . . . . . . . . . . . . . 27

2.2.2 Small-Scale Model . . . . . . . . . . . . . . . . . . 27

2.2 .3 LoS Probability . . . . . . . . . . . . . . . . . 28

2.3 NYU Propagation Channel Model . . . . . . . . . . . . . 28 
2.3.1 Large-Scale Model . . . . . . . . . . . . . . . . . . . . 28

2.3.2 Small-Scale Model . . . . . . . . . . . . . . . . 29

2.3.3 LoS Probability . . . . . . . . . . . . . . . . . 29

2.4 METIS Map-Based Channel Model . . . . . . . . . . . . . . . 30

2.4.1 Large-Scale Model . . . . . . . . . . . . . . . . . . . . 30

2.4.2 Small-Scale Model . . . . . . . . . . . . . . . . 32

2.5 Evaluation of Large-Scale Path Loss Channel Models Below 6

$\mathrm{GHz} \ldots \ldots \ldots \ldots . \ldots \ldots . \ldots \ldots$

2.5.1 Measurement Campaign ............. 32

2.5.2 Preliminary Results . . . . . . . . . . . . . 34

2.6 Evaluation of Large Scale Path Loss Channel Models Above 6

$\mathrm{GHz} \ldots \ldots \ldots \ldots \ldots . \ldots \ldots$

2.6.1 $28 \mathrm{GHz}$ Measurement Campaign .......... . . 35

2.6.2 $73 \mathrm{GHz}$ Measurement Campaign . . . . . . . . . . 36

2.6.3 Preliminary Results . . . . . . . . . . . . 37

2.7 Conclusions . . . . . . . . . . . . . . . 41

3 Unity3D Game Engine $\quad 43$

3.1 Unity Game Engine Description . . . . . . . . . . . . . . . . . 44

3.2 Unity Interface . . . . . . . . . . . . . . . . . . . . . . . . . . . . 44

3.3 Geometry in Unity . . . . . . . . . . . . . . . . . . . . . 46

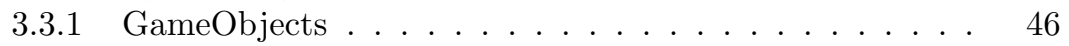

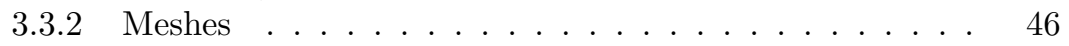

3.4 Lighting Techniques . . . . . . . . . . . . . . . . . . . . 47

3.4.1 Real-time Lighting . . . . . . . . . . . . . . . . . . 47

3.4.2 Baked Lighting . . . . . . . . . . . . . . . . . . . . . . . 47

3.4.3 Pre-Calculated Lighting . . . . . . . . . . . . . . . . . . . . . 47

3.5 Light Types . . . . . . . . . . . . . . . . . . . . . . . . . . . 48

3.6 Light Physics Engine: Ray-Tracing . . . . . . . . . . . . . . 50

3.6.1 Global Illumination . . . . . . . . . . . . . 50

3.6.2 Reflections .................. . . . 52

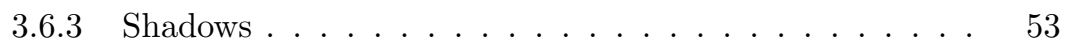

3.7 Object Properties . . . . . . . . . . . . . . . . . . 54

3.7.1 Materials and Shaders ............ 54

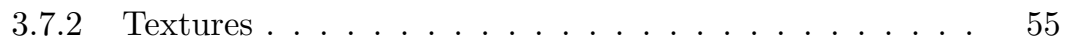

3.8 User Interface . . . . . . . . . . . . . . . . . . . . . . . 55

4 Light Intensity Model for 5G $\quad 57$

4.1 Introduction . . . . . . . . . . . . . . . . 58

4.2 Light Intensity Model . . . . . . . . . . . . . . . . . . . . 58

4.2.1 Concept Details . . . . . . . . . . . . 59 
4.3 Development of the Scenario of New York University in Unity . 60

4.4 Acquisition of Light Intensity Values . . . . . . . . . . . . 61

4.5 Measurement Campaign of Propagation Losses at 28 and $73 \mathrm{GHz}$ at $\mathrm{NYU} \ldots \ldots \ldots \ldots \ldots$. . . . . . . . . . . . . . . 62

4.6 Light Intensity Model Calibration . . . . . . . . . . . . . . . 63

4.7 Model Accuracy . . . . . . . . . . . . . . . . . . . 66

4.8 Computational Complexity . . . . . . . . . . . . . . . . . 69

4.9 Conclusions . . . . . . . . . . . . . . . . . . . 70

5 Extension of the Light Intensity Model $\quad 73$

5.1 Introduction . . . . . . . . . . . . . . . . . 73

5.2 Diffuse Scattering Measurement Campaign at 26 to $40 \mathrm{GHz}$. . $\quad 75$

5.3 Ray-Tracing Calibration . . . . . . . . . . . . . . . 78

5.3.1 Analytical Results . . . . . . . . . . . . . . . 83

5.4 LIM Extension . . . . . . . . . . . . . . . . . . . . . . . . . 92

5.4.1 Light Rendering Process . . . . . . . . . . . . . . . . 94

5.4 .2 Results . . . . . . . . . . . . . . . . . . . . 96

5.4.3 Computational Complexity . . . . . . . . . . . . 101

5.5 Conclusions . . . . . . . . . . . . . . . . . . . . 101

6 Light Intensity Model Evaluation in Indoor Scenarios at $28 \mathrm{GHz} \quad 103$

6.1 Introduction . . . . . . . . . . . . . . . . . . . 104

6.2 Stochastic Channel Models for $\mathrm{mmW}$ in $5 \mathrm{G} \ldots \ldots$. . . . . . 104

6.3 Light Intensity Channel Model _. . . . . . . . . . . . . . 106

6.3.1 Acquisition of Light Intensity Values . . . . . . . . 107

6.3.2 Global Illumination and Light Rendering Process . . . . 107

6.3.3 Light Intensity Model Calibration . . . . . . . . . . . 108

6.4 Evaluation Scenario and Unity Configuration . . . . . . . . . 108

6.5 Evaluation Results . . . . . . . . . . . . . . . . . . . . 109

6.6 Conclusions . . . . . . . . . . . . . . . . . . . . . . . 113

7 A Multi-Purpose Simulation and Visualization Platform for 5G based on Unity $\mathbf{1 1 5}$

7.1 Introduction . . . . . . . . . . . . . . . 116

7.2 Platform Description . . . . . . . . . . . . . . . . . 117

7.2 .1 Block I: External Inputs . . . . . . . . . . . . . . . 118

7.2 .2 Block II: Logical Functions . . . . . . . . . . . . . . 119

7.2 .3 Block III: 3D Representation . . . . . . . . . . . . . . 119

7.2.4 Block IV: Simulation Control . . . . . . . . . . . . 121

7.2 .5 Block V: Presentation . . . . . . . . . . . . . 121 
7.2 .6 Block VI: Outputs . . . . . . . . . . . . . . . . . . . 122

7.3 Use Cases . . . . . . . . . . . . . . . . . . . . . . . . 122

7.3.1 Planning of coverage of mobile networks in the Millimeter

Wave $(\mathrm{mmW}$ ) bands based on Light Intensity Model (LIM) 122

7.3.2 Vehicular Communications Simulation Tool . . . . . . . 125

7.3.3 Ray-Tracing Simulator for $\mathrm{mmW}$. . . . . . . . . . . 127

7.3.4 Unmanned Aerial Vehicle (UAV) Mobile Base Station with Artificial Intelligence Algorithms for Emergency Situations . . . . . . . . . . . . . . . . . 129

7.4 Conclusions . . . . . . . . . . . . . . . . . 130

8 Conclusions and Future Work 131

8.1 Concluding Remarks . . . . . . . . . . . . . . . . . . 131

8.2 Future Research Lines . . . . . . . . . . . . . . . . . . . . . 134

$\begin{array}{ll}\text { Appendices } & 137\end{array}$

A Ray-Tracing Algorithm . . . . . . . . . . . . . . . . 137

A.1 Properties of Dielectric Materials . . . . . . . . . 137

A.2 Free space propagation . . . . . . . . . . . . . . . . 138

A.3 Specular Reflection . . . . . . . . . . . . . . . . 138

A.4 Diffuse Scattering . . . . . . . . . . . . . . . . . . . 139

A.4.1 Lambertian Model . . . . . . . . . . . . . 140

A.4.2 Directive Model . . . . . . . . . . . . . . . . 141

A.5 Diffraction . . . . . . . . . . . . . . 141

$\begin{array}{lr}\text { References } & 145\end{array}$ 


\section{Acronyms}

2D

3D

3G

3GPP

$4 \mathrm{G}$

$5 \mathrm{G}$

AI

AoA

AoD

AR

BRDF

BS

CA

CI

CDF

CPU

CUDA

D2D
Two Dimensions

Three Dimensions

Third Generation

Third Generation Partnership Project

Fourth Generation

Fifth Generation

Artificial Intelligence

Angle of Arrival

Angle of Departure

Augmented Reality

Bidirectional Reflectance Distribution Function

Base Station

Carrier Aggregation

close-in free space reference distance

Cumulative Distribution Function

Central Processing Unit

Compute Unified Device Architecture

Device-to-Device 
DL

ETSI

ER

FDD

GI

GIS

GO

GPU

GPGPU

H2D

H2H

HDRP

HPBW

InH

IMT

IMT-2020
Down Link

European Telecommunications Standards Institute

Effective Roughness

Frequency Division Duplexing

Global Illumination

Geographic Information System

Geometrical Optics

Graphics Processing Unit

General Purpose Graphics Processing Unit

Human-to-Device

Human-to-Human

High Definition Render Pipeline

Half-Power Beamwidth

Indoor Hotspot

International Mobile Telecommunications

International Mobile Telecommunications 2020

IMT-Advanced International Mobile Telecommunications Advanced

IoT Internet of Things

ITU

International Telecommunications Union

ITU-R International Telecommunications Union (ITU)

Radiocommunications Sector

KPI

Key Performance Indicator

LB Light Bounce

LIM Light Intensity Model

LoS Line of Sight 
LSI

M2M

MatSIM

METIS

METIS-II

MIMO

mMIMO

mMTC

mmW

NLoS

NR

NYU

O2I

$\mathrm{O} 2 \mathrm{O}$

OFDM

PDF

PDP

PLE

QoS

RAN

RAT

RMa

RMSE

RSCP
Light Source Intensity

Machine-to-Machine

Multi Agent Transport Simulation

Mobile and wireless communications Enablers for

Twenty-twenty Information Society

Mobile and wireless communications Enablers for

Twenty-twenty Information Society II

Multiple-Input Multiple-Output

massive MIMO

massive Machine Type Communications

Millimeter Wave

Non Line of Sight

New Radio

New York Univeristy

Outdoor to Indoor

Outdoor to Outdoor

Orthogonal Frequency Division Multiplexing

Probability Distribution Function

Power Delay Profile

Path Loss Exponent

Quality of Service

Radio Access Network

Radio Access Technology

Rural Macro-cell

Root Mean Square Error

Receive Signal Code Power 


\begin{tabular}{|c|c|}
\hline RT & Real Time \\
\hline SB & Shadow Bias \\
\hline SGE & Serious Game Engineering \\
\hline SUMO & Simulation of Urban Mobility \\
\hline SS & Shadow Strength \\
\hline TDD & Time Division Duplexing \\
\hline UAV & Unmanned Aerial Vehicle \\
\hline UE & User Equipment \\
\hline UI & User Interface \\
\hline UHD & Ultra High Definition \\
\hline UL & Up Link \\
\hline UMa & Urban Macro-cell \\
\hline UMi & Urban Micro-cell \\
\hline uMTC & ultra-reliable Machine Type Communications \\
\hline UMTS & Universal Mobile Telecommunications System \\
\hline UPM & Unity Package Manager \\
\hline UTD & Uniform Theory of Diffraction \\
\hline V2V & Vehicle-to-Vehicle \\
\hline V2X & Vehicle-to-anything \\
\hline VR & Virtual Reality \\
\hline WINNER & Wireless World Initiative New Radio \\
\hline WRC & World Radiocommunication Conference \\
\hline xMBB & extreme Mobile Broadband \\
\hline
\end{tabular}




\section{Notation}

This section describes the notation used in this Thesis.

$\mathbf{A}$ (boldface capital letter) and $\mathbf{A}_{k}$ represent matrices.

$[\mathbf{A}]_{i j}$ is the element of matrix $\mathbf{A}$ in row $i$ and column $j$.

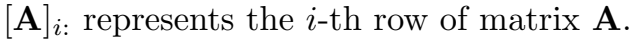

$[\mathbf{A}]_{: j}$ represents the $j$-th column of matrix $\mathbf{A}$.

$\mathbf{A}^{H}$ denotes the conjugate transpose or Hermitian transpose of $\mathbf{A}$.

$\mathbf{a}$ (boldface lower-case letter) and $\mathbf{a}_{k}$ represent vectors.

$[\mathbf{a}]_{i}$ represents the $i$-th element of vector $\mathbf{a}$.

$a$ and $A$ (not boldface italic letters) represent scalars. 



\section{Chapter 1}

\section{Introduction}

This Thesis presents the results of a research activity focused on the field of radio propagation. Specifically, on the use of the lighting models of game development environments for the estimation of the radio channel propagation losses in the frequency bands above $6 \mathrm{GHz}$. The Thesis also deals with the development of serious games for the visualization and simulation of the Fifth Generation (5G) communication systems in super realistic environments. The present chapter contains an introduction to the Thesis in which the motivation of the work carried out is explained and also, the historical background and the most relevant concepts are provided.

To that end, this chapter has been divided into the following sections:

- Section 1.1 presents the historical background of mobile communications and describes the most relevant radio channel concepts within the context of this Thesis.

- Section 1.2 analyses the state of the art in the field of study of this Thesis.

- Section 1.3 identifies the main problem that motivates this Thesis and defines the research hypothesis.

- Section 1.4 defines the research objectives of this Thesis.

- Section 1.5 outlines the structure of the Thesis summarizing the main contents of each chapter.

- Section 1.6 lists the publications related with this Thesis including journals and conference papers. 


\subsection{Background}

This section presents the background and the context in which the Thesis has been developed. This context includes the evolution of mobile communications systems and the standardization process towards $5 \mathrm{G}$ and the main technical concepts of propagation models for $5 \mathrm{G}$ are also discussed.

\subsubsection{Towards 5G}

The first discussions on the new generation of mobile networks, known as $5 \mathrm{G}$, began in 2012. The motivations for $5 \mathrm{G}$ arise from the exponential growth of connections, high traffic demand and communication needs for new emerging scenarios such as Internet of Things (IoT), industry 4.0, or intelligent cities, among others. In fact, several reports in that year estimated that by 2020 the number of connections worldwide would reach 50 billion.

The main entities involved in the standardization process of $5 \mathrm{G}$ are the International Telecommunications Union (ITU) Radiocommunications Sector (ITU-R) and the Third Generation Partnership Project (3GPP):

- The ITU-R is one of the sectors of the ITU and one of its functions is to define standards for mobile and wireless communications systems. It has successfully driven the standardization of Third Generation (3G) and Fourth Generation $(4 \mathrm{G})$ in the past and is now involved in the standardization of $5 \mathrm{G}$.

- The 3GPP is a mobile standards organization composed of seven regional telecommunication organizations and market representative partners. Its work is divided into three fields: radio access networks, aspects of services and systems, and central networks and terminals. In the standardization process of the $5 \mathrm{G}$ the $3 \mathrm{GPP}$ proposes a new radio access technology called 5 GNew Radio (NR). The first version of the specification for 5GNR was completed in 2017. In fact, in 2019, the 3GPP published the Release 15 where it describes Phase 1 of the $5 \mathrm{G}$ standard.

ITU-R introduced, in 2012, the first recommendation on the framework and general objectives for the development of International Mobile Telecommunications (IMT) systems by 2020 [1]. Currently the term International Mobile Telecommunications 2020 (IMT-2020) is used in reference to the standard 5G. This recommendation is the starting point of the $5 \mathrm{G}$ standardization process and allowed the $5 \mathrm{G}$ vision to be established. That vision depicted a system that provides full connectivity where any person or device can be connected wherever they are, at the data rate they want and with minimal latency [2][3]. 
In fact, $5 \mathrm{G}$ is expected to produce a paradigm shift and have a major impact on society and industry. To fulfill that vision, $5 \mathrm{G}$ must enable a next level of communications that allows for interaction between the human and the surrounding environment. And, also, to expand centralized human-to-human communications into Human-to-Device (H2D) and Device-to-Device (D2D) [4].

The above-mentioned recommendation [1], also sets out the main trends at user level, applications and technology. And, presents a study on the technical feasibility for the use of the 6 to $100 \mathrm{GHz}$ bands. This recommendation leads to the consideration of three different scenarios for $5 \mathrm{G}[5]$ :

- extreme Mobile Broadband (xMBB): Provides extreme data rates and low latency communications, and broad coverage that enhances the quality of experience by providing reliable rates in the coverage area. Its use cases are focused on human-centric communications for access to multimedia content, services and data.

- massive Machine Type Communications (mMTC): Provides wireless connectivity to several million network-enabled devices. Connectivity is scalable for an increasing number of devices, wide area coverage and deep indoor penetration are prioritized over maximum rates compared to xMBB . This scenario covers Human-to-Human (H2H) and Machine-toMachine (M2M) communications. It is characterized by use cases where low latency, high reliability and high availability are required. For example, for Vehicle-to-Vehicle (V2V) communications, smart city, smart grid, security, industrial processes (industry 4.0), etc.

- ultra-reliable Machine Type Communications (uMTC): Provides ultra-reliable low latency communication links for network services with extreme requirements for availability, latency and reliability. It is focused on M2M communications, where the main characteristics are the large number of connected devices, the relatively low data, low sensitivity to delay and high battery life.

\subsubsection{G Requirements}

Continuing the standardization process, ITU-R established the requirements and its evaluation methodologies for IMT-2020 systems [1]. The Key Performance Indicators (KPIs) used to set the requirements can be summarized as follows:

- Peak Data Rate [Gbps]: Maximum data rate achievable under ideal conditions by a user or device. 
- User Experienced Data Rate [Mbps o Gbps]: Reachable user/device data rate in the coverage area. This requirement is defined by the ITU at 100 Mbps in Down Link (DL) and 50 Mbps in Up Link (UL).

- Latency[ms]: The contribution of the radio network from the time the source sends a packet until it is received at the destination expressed in milliseconds.

- Mobility $[\mathbf{k m} / \mathbf{h}]$ : The maximum speed at which a defined Quality of Service (QoS) is reached and the transfer between the radio nodes is guaranteed.

- Connection Density[\#devices $\left./ \mathbf{k m}^{2}\right]$ : The total number of connected devices per unit area.

- Network Energy Efficiency [bit/Joule]: Energy efficiency has two aspects. On the network side, it refers to the relationship between the amount of information transmitted or received in bits to users and the energy consumption of the Radio Access Network (RAN). And, on the device side, it refers to the relationship between the amount of information in bits and the power consumption in the communication module. In both cases in bit/Joule.

- Reliability[\%]: The probability of completed transmissions within a required period of time

- Area Traffic Capacity $\left[\mathrm{Mbps} / \mathbf{m}^{2}\right]$ : The total traffic throughput served per geographic area.

The requirements set for these KPIs in IMT-2020 were in some cases absolute, while in others they were relative to the values provided by International Mobile Telecommunications Advanced (IMT-Advanced). Figure 1.1 shows graphically the requirements for IMT-2020 and its relation with IMT-Advanced capabilities.

These KPIs used in 5G definition are not equally important in all the $5 \mathrm{G}$ scenarios. Figure 1.2 illustrates the importance of each KPI in each one of the $5 \mathrm{G}$ scenarios presented in the previous subsection.

\subsubsection{Use Cases}

The three main features of $5 \mathrm{G}$ systems are the high data transfer rate, the number of connected devices it supports, and the ultra-low latency. These features will allow to offer new services and use cases in the main economic 
sectors [6]. In fact, the 5G will extend the typical use cases of previous mobile systems to critical communications applications.

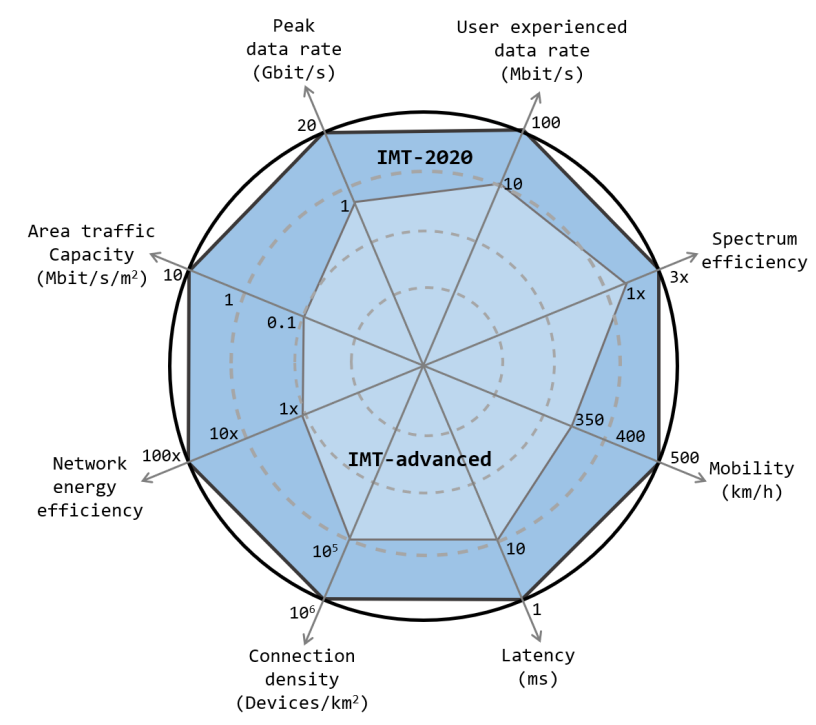

Figure 1.1: 5G requirements defined by ITU-R [7]

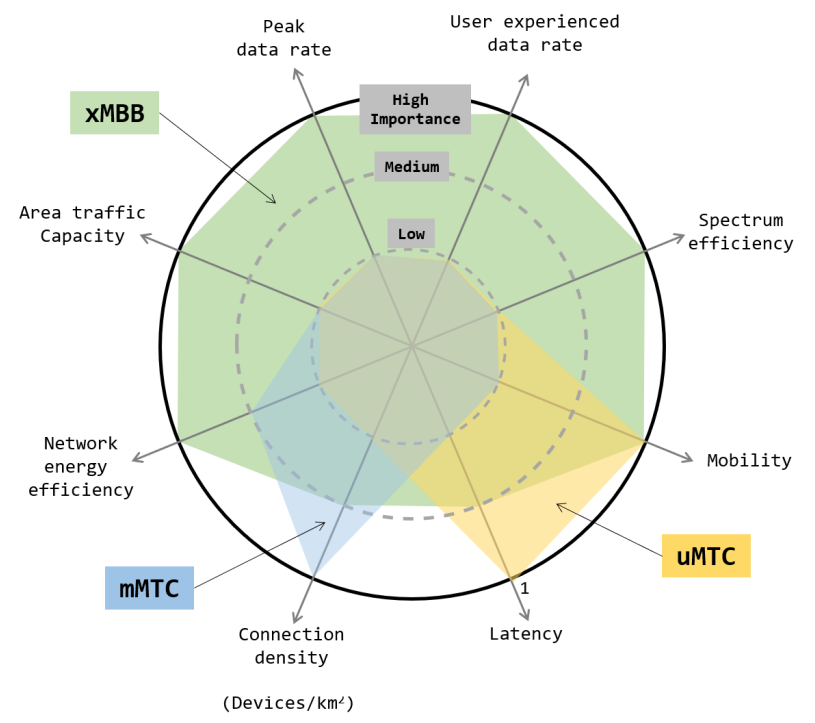

Figure 1.2: $5 \mathrm{G}$ requirements defined by ITU [8] 
The most relevant technical and business use cases defined for 5G are [9]:

- Mobile Broadband: Mobile broadband goes beyond basic internet access, it covers a variety of new services that require high-speed internet with response capability in Real Time (RT) and that support Virtual Reality (VR) and Augmented Reality (AR).

- Vehicular Communications: V2V and Vehicle-to-anything (V2X) communications will make transportation more efficient, comfortable, safe and secure. The $5 \mathrm{G}$ is expected to be the technology that supports massive connectivity between vehicles and road infrastructure on a continuous, reliable basis with minimal latency. Applications in this sector range from synchronizing traffic lights to enabling autonomous cars.

- Smart Cities: This use case focuses on meeting the connectivity requirements of people living in urban areas to improve their quality of life. The $5 \mathrm{G}$ will integrate dense networks of wireless sensors within an intelligent network to become an integrated solution in different sectors such as security, transport, and energy saving, among others.

- Smart Grids: The purpose of smart grids is to control and manage energy sources and energy distribution resources to optimize electricity supply consumption. For example, the use of available renewable energy can be optimized according to demand, time or consumption forecasts. In this use case, the $5 \mathrm{G}$ will be crucial to ensure mass connection of sensors and management of critical infrastructure in RT.

- Industrial Sector: In the industrial sector, reliability and ultra-low latency are essential requirements for communication between machines in the production chain. The purpose of industrial sector automation is to have complete insight, real-time control and intelligent data analysis to enable intelligent and flexible production processes, increase production efficiency and bring production closer to end users. Specifically, for realtime control, a latency of less than $10 \mathrm{~ms}$ and a reliability of $99.999 \%$ are required .In some cases, which involve the collection and reporting of data from industrial meters and sensors, ultra dense D2D communications will be required with massive access capabilities and extremely low power consumption for terminals.

\subsubsection{G Enablers}

In order to achieve the $5 \mathrm{G}$ requirements and make new use cases feasible, the following key enabling technologies have been identified by ITU [10]: 
- Advanced Modulation and Coding Schemes: Given the breadth of applications foreseen for $5 \mathrm{G}$, the criteria for the selection of modulation and coding schemes must fit the type of communication. For example, for M2M communications, a robust and very low-power link is required. Whereas, for real-time communications, high bandwidth and high frequency bands should be considered to prioritize data rate and low latency. Waveforms and advanced modulation and coding schemes are being investigated. The proposed 5G modulation schemes are filter bank multi-carrier, universal filtered multi-carrier, generalized frequency division multiplexing, and filtered Orthogonal Frequency Division Multiplexing (OFDM).

- 3D Beamforming: In most current cellular networks, antenna elements at the base station (BS) are placed along the horizontal axis. 3D beamforming adds beamforming capabilities on the vertical axis and provides better inter-cellular interference management. Therefore, it achieves a higher capacity gain compared to Two Dimensions (2D) beamforming [11]. It is expected that Three Dimensions (3D) beamforming specification improvements will continue in 3GPP releases 14 and 15 .

- Massive MIMO: The main difference between Massive Multiple-Input Multiple-Output (MIMO) and MIMO is the large number of antennas at the base station. With massive MIMO, significant gains can be obtained from improved beamforming. More users can be served in parallel [12], and an increase in spectral efficiency can be experienced [13]. Massive MIMO implies new challenges, such as avoiding pilot contamination. On the other hand, the use of the bands above $6 \mathrm{GHz}$ allows to pack arrays of hundreds of antenna elements in small form factors.

- Flexible Spectrum Usage: The limited use of spectrum requires a flexible use to provide solutions to traffic demand in the different scenarios of IMT-2020 networks. The flexible use of spectrum can improve spectral efficiency through the use of cognitive radio techniques, authorized shared access, joint management of multiple Radio Access Technologys (RATs), aggregation of discontinuous band carriers, including aggregation of inter-band carriers, which could enable Time Division Duplexing (TDD)-Frequency Division Duplexing (FDD) joint operation.

- Simultaneous Transmission and Reception: Also known as fullduplex, is a technique that improves the spectral efficiency and capacity of cellular networks and reduces latency levels. Full-duplex communication is considered a way to potentially double the speed of wireless 


\section{CHAPTER 1. INTRODUCTION}

communication and is considered a key technique for $5 \mathrm{G}$ systems. In theory, full-duplex allows a node to simultaneously transmit and receive in the same frequency band. A limitation on the full-duplex node is selfinterference, i.e., the interference generated by the transmitted signal to the received wanted signal on the same node [14].

- Flexible Back-Haul: This technology increases spectral efficiency and performance in point-to-point and point-to-multipoint links. It is focused on industrial applications and M2M communications. It is characterized by the flexible use the system resources, in a dynamic topology that allows to adapt to different levels of traffic and capacity of the link, and an efficient use of the spectrum.

- Dynamic RAN: In this new RAN generation, the wireless device exhibits a duality, being able to act as both a terminal and an infrastructure node. Dynamic RAN incorporates ultra-dense networks and multi-nodes, and supports direct device-to-device communication for both local and back-haul traffic.

- Technologies to Enhance User Experience: Enabling technologies for broadcast and multicast applications need to achieve ultra-low latencies, high reliability, and high bandwidth. One of the concerns is to improve communications at the edge of the cell, where the use of beamforming with large-scale antennas is proposed [15]. On the other hand, to improve the broadcasting of high quality video one requires encoding technologies that support $4 \mathrm{~K}$ services and virtual reality. Other enabling technologies identified at this point correspond to improvements in positioning and on the use of small-cells to enhance the QoS of users by decreasing the number of users per cell.

\subsubsection{G Spectrum Requirements}

The bands used in $5 \mathrm{G}$ predecessor generations range from $450 \mathrm{MHz}$ to $6 \mathrm{GHz}$. $5 \mathrm{G}$ networks, instead, will have to operate at frequencies below and above $6 \mathrm{GHz}$ to satisfy the requirements of IMT-2020. It is expected that the lower bands will be reused for long-range solutions in 5G networks, and upper bands will be exploited in the order of Millimeter Wave ( $\mathrm{mmW}$ ) bands for mediumand short-range $5 \mathrm{G}$ networks solutions $[16,17]$. The reason is that, although in the bands above $6 \mathrm{GHz}$ there is a large amount of spectrum available, these bands have a higher propagation loss and a higher attenuation due to the blocking of objects compared to bands below $6 \mathrm{GHz}$. This fact does not allow the use of these bands for wide area coverage and this spectrum is considered 
to satisfy the $5 \mathrm{G}$ requirements for extreme data rates and localized areas [18]. On the other side, in the bands below $6 \mathrm{GHz}$ there is over-used and congested spectrum, but they are necessary for the deployment of mobile networks in large environments where wide coverage is required.

While many mmW bands are already being used for back-haul links and short-range services, its usage to provide mobile communications services is still under study. In this regard, according to [19], there are three main factors that affect spectrum selection. First, the spectrum candidate must be available in all or most regions globally. Second, contiguous spectrum is required to provide more flexibility in spectrum management strategies. Third, the propagation characteristics of the spectrum candidate must satisfy the requirements for mobile communications.

ITU plays a leading role in radio spectrum management and in the development of globally applicable standards for radio spectrum IMT-2020. The World Radiocommunication Conference (WRC) 2015, led by ITU, focused on identifying candidate bands for networks below $6 \mathrm{GHz}$. Within all bands, the following bands of special interest for the $5 \mathrm{G}$ were identified [18]. First, a global band identified in many countries, but not in Europe nor in North America in the range 3300-3400 MHz. Second, a global C-band band for all countries in 3400-3600 MHz. Third, a global C-band band identified in many countries, but not in Africa and some counties in Asia-Pacific in 3300-3400 MHz. Finally, a C-band band identified for a few countries in Asia-Pacific in 4800-4990 MHz. Indeed, the use of C-band is expected to provide high bandwidth per operator and to be adequate to meet the traffic requirements of the $5 \mathrm{G}$ in urban, dense urban and high coverage scenarios [19]. On the other hand, there is a lot of interest in the use of $700 \mathrm{MHz}$ band. However, its use for $5 \mathrm{G}$ requires additional work to migrate existing services in these bands [16].

Another central issue discussed at the WRC 2015 was to identify the frequency bands above $6 \mathrm{GHz}$ for the $5 \mathrm{G}$ mobile services, which will be studied at the next WRC 2019. The main bands identified were: $24.25-27.5 \mathrm{GHz}, 37-40.5$ GHz, $42.5-43.5 \mathrm{GHz}, 45.5-47 \mathrm{GHz}, 47.2-50.2 \mathrm{GHz}, 50.4-52.6 \mathrm{GHz}, 66-76 \mathrm{GHz}$, and $81-86 \mathrm{GHz}$.

In this Thesis the focus is precisely on the study of the bands above $6 \mathrm{GHz}$. From the three spectrum requirements for $5 \mathrm{G}$, i.e. (i) availability and consensus at the global level, (ii) contiguous spectrum with large available bandwidth, and (iii) suitable radio propagation. This Thesis focuses on the last point. Specifically, the idea is to provide means to easily model the propagation in bands above $6 \mathrm{GHz}$. Given that the exact bands associated to $5 \mathrm{G}$ above $6 \mathrm{GHz}$ are not yet specified, the study will try be as generic as possible. 


\subsubsection{G Channel Model Requirements and Propagation Scenarios}

In this section, the main channel model requirements for $5 \mathrm{G}$ and the most relevant propagation scenarios are briefly presented to show which are the expectations from any activity in the $5 \mathrm{G}$ channel modeling area.

\section{Channel Model Requirements}

The main factors that determine channel propagation modeling are two according to [20]. The first corresponds to the technological components that provide the service to the end user. From this technological point of view, the requirements of the channel model can be summarized in the following points:

- Radio channel model must support a wide frequency range up to $100 \mathrm{GHz}$ and high bandwidth. In addition, it must include the inherent characteristics of propagation in different frequency bands.

- Channel model must support large dynamic bandwidths ranging from $10 \mathrm{MHz}$ to $2 \mathrm{GHz}$ and must support Carrier Aggregation (CA).

- Channel model must be adapted to the mobility requirements defined by the ITU-R, up to $500 \mathrm{~km} / \mathrm{h}$.

- Channel model should support large sets of antennas, especially for the millimeter-wave bands, where the use of massive MIMO (mMIMO) is very likely.

- The new channel model must ensure spatial, temporal and frequency coherence.

- The model must also ensure that channel states, such as Line of Sight (LoS) and Non Line of Sight (NLoS), for indoor/outdoor locations are implemented.

\section{Propagation Scenarios}

The second factor that determines the channel propagation modeling is the propagation scenario or environment. Propagation scenarios identified for 5G technology are based on their use cases described in Section 1.1.3. The scenarios recommended by the ITU in report [1] classified as:

- Urban Micro-cell (UMi): This is an urban environment, where the base station is located below the roof of the surrounding buildings. The typical 
coverage of this scenario is in the order of 50 to 100 meters. Generally, stochastic propagation models for this type of scenario consider both equations for connections Outdoor to Outdoor $(\mathrm{O} 2 \mathrm{O})$ and Outdoor to Indoor (O2I).

- Urban Macro-cell (UMa): This scenario is similar to UMi, the difference is that the base station is located on the rooftop and above the surrounding buildings. The typical coverage of this scenario is over 100 meters.

- Indoor: The main feature of this scenario is that the base station is located inside a building. This scenario includes environments such as offices, shopping malls, corridors and others. The typical height of the base station is between 2 and 3 meters.

- Backhaul: This scenario refers to the connection between two base stations, usually a combination of UMi or UMa with pico or fento cells.

- D2D: This scenario refers to the communication between devices in open areas, streets and indoor scenarios. V2V is a special case, where the main feature is mobility.

In this Thesis the focus is on urban micro-cell and urban macro-cell scenarios. Concerning the above mentioned technical requirements, in this Thesis more emphasis is put on ensuring an accurate channel modeling in LoS and NLoS, and for a wide frequency range. The reason for ignoring other requirements is that the Thesis focuses on the prediction of the large scale propagation losses.

\subsection{State of the Art Analysis}

This section presents an analysis of the state of the art within the field of study of this Thesis. This analysis is divided into three parts. The first part details the concepts related to the radio channel of $5 \mathrm{G}$. The second presents the channel models available for frequencies above $6 \mathrm{GHz}$. The third part focuses on the Serious Game Engineering (SGE), their classification and their use in applications for wireless communications.

\subsubsection{G Radio Propagation Channel}

The radio channel is the environment where radio waves propagate from a transmitter to a receiver. This environment may contain only free space, but it can also be a complex environment with different objects interacting with 
radio waves, e.g. buildings, trees and vehicles [21]. Figure 1.3 shows some of the main phenomena that affect radio propagation: free space propagation losses, penetration through materials, reflections, diffraction and scattering. Other relevant phenomena are atmospheric attenuation and rain losses.

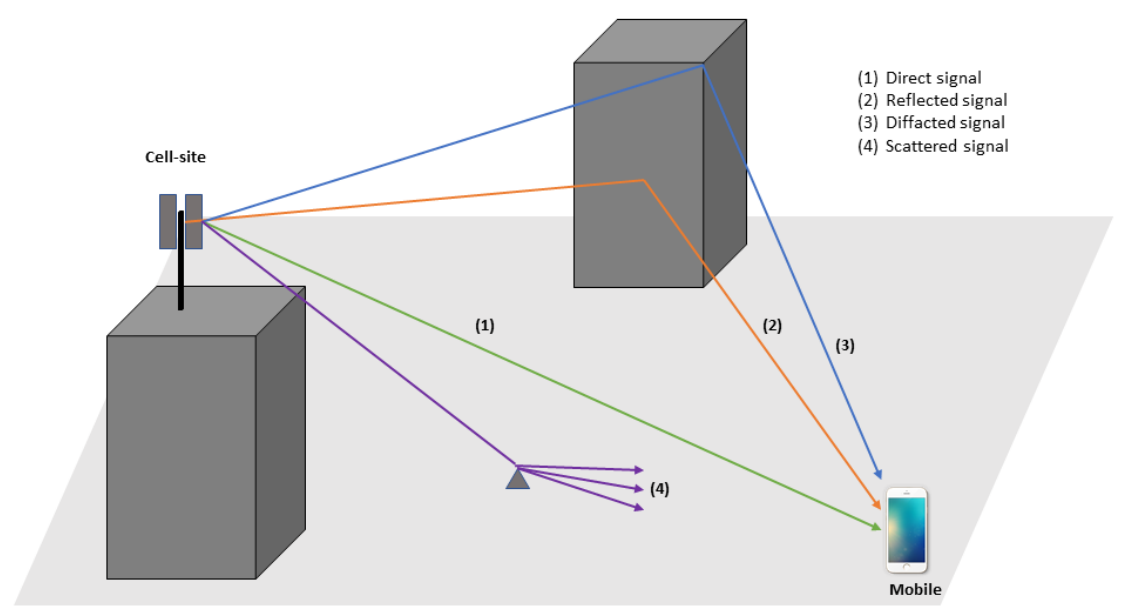

Figure 1.3: Multi-path propagation. (1) direct signal, (2) reflected signal, (3) diffracted signal, (4) scattered signal

Each phenomenon produces changes in the phase and power of the radio waves which eventually produce a variation of the channel in time, frequency and space. The effect of each phenomenon on the radio waves depends on the frequency of those waves. For example, one of the main differences between $\mathrm{mmW}$ frequencies and frequencies below $6 \mathrm{GHz}$ is the sensitivity to signal blockage which is much higher above $6 \mathrm{GHz}$. Below is a brief description of each phenomenon, more details can be found in Appendix A.

\section{A. Reflection}

Reflection occurs when an electromagnetic wave strikes an object that has a larger size than the wave wavelength [22]. For 5G networks, operating above $6 \mathrm{GHz}$, the radio wave will be reflected in most objects in both indoor and outdoor environments, due to the shorter wavelength. The reflection depends on multiple characteristics such as the wave frequency, the electrical and geometrical characteristics of the object, the angle of incidence, etc. 
Focusing on the frequency, studies of the reflections in the $6 \mathrm{GHz}$ bands have shown interesting results. For example, in [23], an analysis of reflection and penetration is made for different materials commonly found in indoor and outdoor scenarios. Losses due to penetration were high for brick materials, $28.3 \mathrm{~dB}$, and for tinted glass there was no noticeable signal penetration. Other materials, such as drywall and transparent glass presented lower penetration losses of $6.84 \mathrm{~dB}$ and $3.6 \mathrm{~dB}$, respectively. Due to the fact that there has to be an equilibrium of signal power, reflection takes on greater importance in materials where the attenuation by penetration is lower, i.e. the reflection coefficient is higher for bricks and tinted glass than for drywall or transparent glass. Therefore, in [23] it is concluded that the reflections are more relevant in outdoor scenarios due to a greater presence of materials with high reflection coefficients, such as bricks and tinted glass, among others, compared to indoor scenarios where is more common to find drywall, for example.

An extension of reflection studies for the $38 \mathrm{GHz}$ band [24], concludes that in UMa and UMi scenarios the power levels due to specular reflections are adequate for the estimation of propagation losses. Nevertheless, it is emphasized that the contribution due to the reflections can change considerably from one scenario to another depending on the density of obstacles. The reason is that, due to the higher number of obstacles, the contribution of the reflections will be more relevant than in non-dense scenarios.

\section{B. Diffuse Scattering}

Diffuse scattering can be described as a special case of reflection that occurs when a radio wave strikes irregular objects whose irregularities are smaller than the radio wavelength. Although the intensity of the scattered rays is usually lower than the intensity of the reflected signal they can contribute significantly to the total received power [22]. Diffuse scattering has been heavily researched for frequencies below $6 \mathrm{GHz}$. However, for communications in relatively small environments, or where the number of obstacles is small, diffuse scattering components are less dominant than specular components [25]. On the other hand, for bands above $6 \mathrm{GHz}$, diffuse scattering components take on greater importance [26]. Typically, in order to estimate diffuse scattering, Effective Roughness (ER) and Beckmann-Kirchhoff models are used. ER introduces two scattering pattern models: Lambertian Model and Directive Model. The Lambertian Model presents maximum power in the direction perpendicular to the reflection surface without considering the direction of incidence 


\section{CHAPTER 1. INTRODUCTION}

wave [27]. This model introduces an $S$ coefficient that is related to the part of energy of the impinging wave contained in the scattering components. The Directive Model and the Beckmann-Korchoff model are similiar: the maximum scattering lobe is steered to the direction of specular reflection.

In [28], Jürvelüinen et al., conducted a study for the estimation of diffuse scattering characteristics in the frequency of $60 \mathrm{GHz}$. In this study, a parameter $S$ of directive scattering losses was calibrated for indoor scenarios, and also, small-scale parameters were calculated including diffuse scattering components. The results show an average $S$ of 0.5 , a Root Mean Square Error (RMSE) of the delay estimate of $8.2 \%$, and errors of the azimuth and elevation of $2.6^{\circ}$ and $0.6^{\circ}$ respectively. Recent studies on terahertz bands indicate that diffuse scattering components have a strong impact on NLoS communications [29]. Poesiewicz et al., in [30], demonstrate that in very rough surface diffuse scattering dominates the specular reflection behavior and that not considering diffuse scattering could lead to errors between $1.5 \mathrm{~dB}$ to $15.2 \mathrm{~dB}$ at $350 \mathrm{GHz}$ in a simple room scenario.

\section{Diffraction}

Diffraction is based on the Huygens Law, which states that any point on an initial wavefront can be considered as a source of secondary spherical waves that extend in all directions with the same speed, frequency and wavelength as the wavelength from which they originate [31]. The signal strength of the diffracted rays will, in general, be lower compared to the reflected signal [32].

\section{Atmospheric and Rain Attenuation}

Another aspect to consider in the radio channel for the $5 \mathrm{G}$ is the attenuation due to rain and the atmospheric absorption characteristics of the mmW communications. In the study conducted in [33], it is indicated that atmospheric absorption for $28 \mathrm{GHz}$ and $38 \mathrm{GHz}$ is only $0.06 \mathrm{~dB} / \mathrm{km}$ and $0.08 \mathrm{~dB} / \mathrm{km}$ respectively, and for the $70-90 \mathrm{GHz}$ bands it is around $0.12 \mathrm{~dB} / \mathrm{km}$. In another study, in [34], a measurement campaign was carried out for bands above $10 \mathrm{GHz}$ in order to measure the rain attenuation, and it was concluded that the attenuation is not significant. Similarly, it can be seen in the graphs of the estimation of losses due to atmospheric gases available in the ITU recommendation [35], where atmospheric attenuation is not significant for wireless communications below $100 \mathrm{GHz}$ and for links below $5 \mathrm{~km}$. 


\subsubsection{Propagation Channel models above $6 \mathrm{GHz}$}

Propagation channel models are indispensable for estimating interference, assigning frequency and establishing cell parameters in mobile communications systems. Indeed, the knowledge of the radio channel allows an adequate planning of the wireless networks [2]. New networks shall exploit potential new spectra over $6 \mathrm{GHz}$ [4], so it is essential to develop reliable channel models that allow a correct characterization of the radio channel in different scenarios [36].

\section{Channel Model Classification}

Channel models can be classified into two large groups: deterministic models and stochastic models. Deterministic models are those that take into account the structure and geometry of the propagation environment. Their objective is to reproduce all the geometric and electromagnetic parameters associated with a particular scenario. These models can be pure deterministic like the general ray-tracing model or semi-deterministic, like the map-based channel model of Mobile and wireless communications Enablers for Twenty-twenty Information Society (METIS) [20]. Stochastic or probabilistic channel propagation models are based on probability density functions that define the type of communication for each environment or scenario, they can be divided into stochastic models based on geometry or pure stochastics. The former are widely used by $3 \mathrm{GPP}$ and IMT-2020 as channel models above $6 \mathrm{GHz}[7,37]$. See a diagram that illustrates this classification in Figure 1.4.

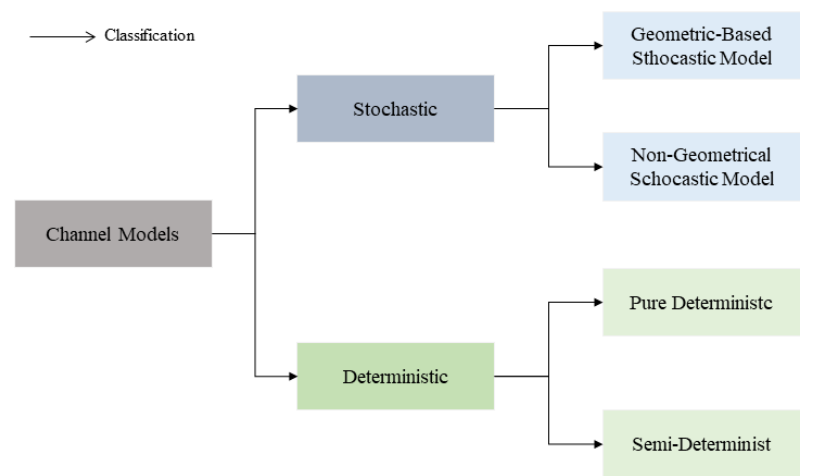

Figure 1.4: Channel model classification diagram

The above mentioned types of models present different approaches to model the path losses. In the following, this specific part of the channel modeling is 
detailed for each model category and exemplary models developed by different organizations are presented.

- Stochastic Models: In this case, the path loss models are very simple and consist basically in functions with one or several constant factors and a term that depends on the logarithm of the distance between the transmitter and the receiver. In some cases, these functions may be different for LoS and NLoS situations. For a specific position, probabilistic functions are used to obtain the probability of being in line of sight for a given distance between transmitter and receiver [38]. One of the advantages of these models is their computational efficiency due to its simplicity. Its main disadvantage is their inaccuracy, for example to predict losses in real environments. Some relevant models of such kind for $5 \mathrm{G}$ are the the 3GPP model [37], IMT-2020 model [39] and the NYU model [40].

1. 3GPP: The model proposed by the $3 \mathrm{GPP}$ is valid for frequencies above $6 \mathrm{GHz}$ up to $100 \mathrm{GHz}$. The model is complete and includes several scenarios such as Rural, UMi, UMa and Indoor - shopping mall. It is suitable for back-haul, D2D and V2V communications.

2. mmMAGIC: It is a probabilistic model that is designed for frequencies from $6 \mathrm{GHz}$ to $100 \mathrm{GHz}$. It is based on the 3GPP model, and performs an extension to improve the accuracy of the channel modeling. It is based on extensive measurement campaigns carried out in Rural, UMi, UMa and Indoor scenarios for the entire frequency range.

3. New York Univeristy (NYU): The NYU propagation loss model, described in [40], is an extension of the channel propagation models proposed by the $3 \mathrm{GPP}$ with minor modifications. The NYU model is valid for frequencies in the range of $6 \mathrm{GHz}$ to $100 \mathrm{GHz}$. Its large potential is based on the extensive measurement campaigns carried out in the frequency range from $2 \mathrm{GHz}$ to $73 \mathrm{GHz}$ in UMa, UMi, and Indoor Hotspot ( $\mathrm{InH})$ scenarios. The advantage of NYU extensive measurement campaigns is that it is suitable for a propagation loss model that fits the evaluation scenario. In [41], probability functions for two scenarios, a shopping mall and an open plaza, are compared. The results show that the exponential model of the NYU, defined in [40], has improved performance with respect to the functions of IMT-2020 and 3GPP.

- Deterministic Models: Deterministic models are based on a detailed description of the propagation environment and antenna configuration. 
The purpose of deterministic models is to recreate the geometry and electromagnetic parameters of propagation environments such as streets, buildings, trees, offices, desks, etc. It is for this reason that deterministic models are geometrically and computationally complex, however, they are the most accurate models. For example, ray-tracing is a pure deterministic model that considers the electromagnetic properties of materials, the geometry and positioning of all the elements of the scenario. It is an accurate model with detailed angular information and is suitable for the exhaustive estimation of channel variables for both large and small scale fading [42]. For 5G, several deterministic models were proposed that attempt to simplify computational cost:

1. IEEE 802.11ad: This semi-deterministic model is oriented to work in short range communications, in the $60 \mathrm{GHz}$ band. For the modeling of propagation losses the specular components are calculated by ray-tracing algorithms, and the components due to diffractions, diffuse scattering and transmission are aggregated in a stochastic way.

2. MiWEBA: This model is an extension of the IEEE 802.11ad model that focuses on back-haul and D2D communications. It is designed for frequencies above $6 \mathrm{GHz}$ up to $72 \mathrm{GHz}$. A characteristic of this model is that the specular components are modeled deterministically and the others stochastically. The available scenarios for this model are open area, street canyon, above roof top and D2D scenarios.

3. METIS: METIS model is a semi-deterministic map-based model based on measurement campaigns for bands from $6 \mathrm{GHz}$ to $70 \mathrm{GHz}$ [20]. In this model, eight different scenarios are detailed among which are UMa, UMi, indoor, shopping mall, street canyon. The model uses ray-tracing techniques to obtain large-scale fading characteristics for a specific site environment, and results based on measurements to model small-scale fading statistics. Small-scale statistics are generated following 3GPP and Wireless World Initiative New Radio (WINNER) modeling approaches [43].

\section{Measurements Campaigns Above $6 \mathrm{GHz}$}

The characterization of the channel at mmW frequencies has been carried out by exhaustive measurement campaigns in different scenarios. Most studies focus on the UMa, UMi and indoor scenarios, which are typically where mobile networks are implemented. For example, in [44], broadband channels were studied for communications in an urban environment in downtown Denver in 
9.6 GHz, $28.8 \mathrm{GHz}$ and $57.6 \mathrm{GHz}$ frequency bands. In [44], measurements were taken in the center of Oslo, at $59 \mathrm{GHz}$, in a typical urban environment with streets of different dimensions, buildings and trees. Several measurement campaigns have been conducted in the $60 \mathrm{GHz}$ bands in UMa environments [45-48]. Rappaport and colleagues led an extensive measurement campaign at $28 \mathrm{GHz}, 38 \mathrm{GHz}, 60 \mathrm{GHz}$ and $73 \mathrm{GHz}$ on the Manhattan campus of NYU. For outdoor campaigns they considered the $28 \mathrm{GHz}, 38 \mathrm{GHz} 60 \mathrm{GHz}$ and $73 \mathrm{GHz}$ bands [49, 50] and for indoor $28 \mathrm{GHz}$ and $73 \mathrm{GHz}$ bands [51-53]. For each measurement campaign performed, they provided the parameters of the measurement environment such as the geographical coordinates of Tx and Rx for outdoor scenarios and location plans for indoor scenario and the measurement results. The availability of results for different bands and the widely available characterization of Manhattan scenario makes this measurement campaign very interesting to be used for the calibration of new propagation models.

\subsubsection{Serious Game Engineering}

\section{Serious Game Engineering Definition}

SGE is the use of game engines for non-entertainment purposes. Serious games are not a particular game genre [54]. In that sense it is necessary to establish the difference between SGE and entertainment games. The first big difference between entertainment games and SGEs is their focus, the first are focused on fun and creating a rich user experience. Meanwhile, SGEs are oriented towards solving a particular problem and establishing learning-oriented mechanisms. The second difference lies in the way the game works: entertainment games seek to make the game intuitive, and that users be focused on the fun parts of the game and not on the game criteria details. On the other hand, the SGE wants users to make conscious decisions and not random decisions. Table 1.1 summarizes the main differences between entertainment games and SGE.

In the SGEs' performance areas, education ranks first. In fact, studies show that gaming-based learning is more effective than traditional methods [56]. However, education is not the only goal of SGEs. There is a wide range of SGEs aimed at the health sector, business management, industrial safety, defense, aeronautics, and engineering.

\section{Serious Game Engineering Analysis}

An accepted model for the classification of SGE is the Gameplay/Purpose/Scope (G/P/S) model, proposed by [57]. This model uses a set of criteria that extends the "Purpose \& Market" paradigm by the addition 
Table 1.1: Difference between entertainment game and serious game [55]

\begin{tabular}{|l|l|l|}
\hline & $\begin{array}{l}\text { Entertainment } \\
\text { Games }\end{array}$ & Serious Games \\
\hline $\begin{array}{l}\text { Task vs rich experi- } \\
\text { ence }\end{array}$ & $\begin{array}{l}\text { Rich experiences } \\
\text { preferred }\end{array}$ & $\begin{array}{l}\text { Problem solving in } \\
\text { focus }\end{array}$ \\
\hline Focus & To have fun & $\begin{array}{l}\text { Important elements } \\
\text { of learning }\end{array}$ \\
\hline Simulations & $\begin{array}{l}\text { Simplified simula- } \\
\text { tion process }\end{array}$ & $\begin{array}{l}\text { Assumptions neces- } \\
\text { sary for workable } \\
\text { simulations }\end{array}$ \\
\hline Communication & $\begin{array}{l}\text { Communication is } \\
\text { often perfect }\end{array}$ & $\begin{array}{l}\text { Should reflect natu- } \\
\text { ral (i.e., non perfect } \\
\text { communication) }\end{array}$ \\
\hline
\end{tabular}

of a "Gameplay" related criteria. The great potential of the game engines allows to create applications with super realistic environments and in real time. They even allow to easily integrate algorithms of artificial intelligence and augmented reality. Therefore, the applications that can be created under the SGE criteria, are unlimited. In fact, games and simulators based on SGE have been developed for multiple purposes.

\section{Serious Game Engineering Benefits}

The most important benefits of SGE are:

- SGE allows users to experience situations that it would otherwise be impossible to experience in real life owing to aspects related to costs, resources, time, security, among others.

- SGE allows a greater acquisition of knowledge, this means that SGE is more effective than traditional methods by using audiovisual media for the training and learning process.

- SGE allows to reduce the costs in the training, planning and implementation of technologies through the advanced use of simulators based on real situations. 
- SGE motivates users to complete learning activities and provides them with authentic learning experiences during which entertainment and learning are seamlessly integrated.

\section{Serious Game Engineering and Unity3D}

Unity game engine, is a 3D graphic and game engine that comes packaged as a tool to create games, interactive applications, visualizations and animations in 3D and real time. In addition, Unity can deploy content for multiple platforms such as PC, Mac, Nintendo Wii and iPhone. The Unity3D editor is the centerpiece of the production line, offering a complete visual editor for creating games. The game content is built from the editor and the gameplay is programmed using a scripting language [58].

The use of the Serious Games concept in Unity3D is increasing, due to its high potential for 3D content development. Currently, successful applications are being developed with 3D content for military simulation, medical training, virtual reality, architecture, and advertising, among others. Several applications and research developed using SGE and Unity engine show promising results [59-61]. With these results, it is possible to consider the use of Unity in applications oriented to the area of mobile communications. In fact, one of its main advantages is considered, the ability to implement virtual environments, where it is possible to design in detail a scenario that includes buildings, streets, green areas and even patterns of vehicular and pedestrian traffic that replicate the characteristics and conditions of a real scenario [62]. Another equally important advantage is its powerful light engine, which not only allows us to obtain real visual results, but also enables the simulation of the main phenomena associated with light such as diffraction, reflection, refraction, among others. This capacity facilitates the simulation of the propagation of millimeter waves in virtual environments and in real time for the fifth generation mobile communications systems.

Considering this and other studies and investigations, we can formulate the hypothesis that it is possible to simulate the propagation of signals using video game platforms with Serious Game Engineering techniques.

\section{Serious Game Engineering About Wireless Communications}

Currently, given that Serious Game Engineering is in full growth, there is little literature with few applications developed in the area of mobile communications. However, we can find in [63] an example of the development of a basic tool for planning mobile networks using "Serious Game", which allows some measurements of propagation losses and estimation of basic KPIs in a virtual scenario. The same author, in [64], compared the propagation and delay 
loss measurements made on the $4.2 \mathrm{GHz}$ band, which were compared with the ray-tracing simulator based on the gaming engine of Jmonkey. In [65], a complex urban scenario has been implemented within the game engine to calculate propagation losses, the ray-tracing algorithm implemented allowed one reflection and two diffractions at the edges of buildings. The calculation time was $280 \mathrm{~min}$ throwing rays every $0.27^{\circ}$. The average error obtained was $4.05 \mathrm{~dB}$. One of the disadvantages is computational cost. Nevertheless, in [66], it is stated that it is possible to improve the simulation time of the ray-tracing implemented in the Graphics Processing Unit (GPU) using Compute Unified Device Architecture (CUDA) or OpenCL. In the same line, in [67], a ray-tracing model has been developed based on games engine for the estimation of propagation losses and the estimation of delay spread in indoor environments for frequency of $594 \mathrm{MHz}$.

In [68], an application has been developed to simulate a traffic network based on Unity's game engines. The purpose of this project, called Veneris, is to implement a vehicular network simulator that includes traffic dynamics, a realistic environment and simulation of the propagation losses with a raytracing channel model implemented in the GPU.

In the field of visualization, Mobile and wireless communications Enablers for Twenty-twenty Information Society II (METIS-II) has developed a 5G networks simulator in the Unity's game engine. This simulator recreates an urban scenario of Manhattan grid where it allows to interact in real time with the user and obtain the KPIs of the system, maps of propagation losses, pedestrian and vehicular mobility traces generated with Simulation of Urban Mobility (SUMO), among others [69]. In fact, the objective of the METIS-II is to generate a visualization platform to enable the representation of $5 \mathrm{G}$ concepts that are easy to understand, and that include the proposed use cases for $5 \mathrm{G}$. To create an immersion within the platform, virtual reality has been implemented within the capabilities of the platform [70].

\subsection{Problem and Thesis Scope}

One of the keys to the development and implementation of the new $5 \mathrm{G}$ technology is to solve one of the main problems associated with the new wireless communication technologies, that is the propagation of the radio channel. For this reason, it is of interest to evaluate the propagation in different environments that include dense urban, urban and indoor scenarios

The problem presented has motivated the definition of several hypotheses: 
- Hypothesis 1: The propagation model based on light engines minimizes the error in estimating propagation losses in the millimeter bands compared to other models.

- Hypothesis 2: The implementation of complex 3D scenarios in Unity3D improves the process of estimating propagation losses.

- Hypothesis 3: Light Intensity Model (LIM) is computationally efficient compared to complex ray-tracing models.

- Hypothesis 4: LIM can implement the phenomena related to radio propagation such as transmission, reflection and diffuse scattering.

- Hypothesis 5: Unity's Game Engine allows a complete simulation and visualization of $5 \mathrm{G}$ systems.

\subsection{Thesis Objectives}

This thesis focuses on the study of wireless propagation losses for mobile systems $5 \mathrm{G}$, as well as the simulation of realistic systems for better visualization of $5 \mathrm{G}$ networks. The main objective is to use the Unity's Game Engine for a rapid estimation of radio wave propagation losses and to use the game engine platform as a general simulation interface.

The main objective of the research can be divided into several sub-objectives listed below:

- To investigate the feasibility of Unity3D for the visualization of the simulation of radio propagation of $5 \mathrm{G}$ systems.

- To develop and validate an interface to model the radio propagation in indoor and outdoor environments using realistic environments and light engines.

- To optimize the transfer function to minimize the propagation estimation error compared to other complex ray-tracing tools and others models proposed by 3GPP, IMT-2020 and METIS.

- To expand the capabilities of game engine platforms for the complete simulation of $5 \mathrm{G}$ system using the concept of "serious game engineering". 


\subsection{Thesis Outline}

This Thesis is organized in seven chapters and two appendices as described below:

Chapter 2 describes the proposed radio propagation models for $5 \mathrm{G}$ and includes the channel requirements, a description of the propagation scenarios and the different propagation models available in the literature.

Chapter 3 describes the main features of the Unity3D gaming platform, which includes a description of the features and benefits of its light engine and lighting in real time and the main features of the platform. In addition, the use of Unity3D under the concept of "Serious Game Engineering".

Chapter 4 describes the propagation model developed for 5G systems based on light intensity, explaining in detail the model, the calibration process and the results obtained.

Chapter 5 presents an extension of LIM valid for estimating diffuse scattering for different materials. LIM extension is calibrated with a measurement campaign carried out in our laboratory and compared with ray-tracing algorithms.

Chapter 6 presents the evaluation of the LIM extension model with a measurements campaign in an indoor scenario for $28 \mathrm{GHz}$ and its comparison with stochastic path loss models. The results obtained corroborate the validity of the assessed approach to model propagation losses in indoor scenarios.

Chapter 7 describes the simulation and visualization platform developed in this Thesis and based on Unity as a tool under the concept of "Serious Game Engineering". Several use cases are introduced within this platform for the $5 \mathrm{G}$, ranging from radio planning to use cases with vehicular communications.

Chapter 8 presents the conclusions of the Thesis and describes the future lines of research.

To complement the Thesis, one appendix is presented:

Appendix A describes the ray tracing channel model implemented in Unity game platform. 


\subsection{Thesis Publications}

The work developed during this Thesis made possible the following publications.

\section{Journals}

[J1] J. F. Monserrat, S. Inca, J. Calabuig, and D. Martín-Sacristán, "MapBased Channel Model for Urban Macrocell Propagation Scenarios," International Journal of Antennas and Propagation, vol. 2015, pp. 1-5, Apr. 2015.

\section{Conferences}

[C1] S. Inca, D. Prado, D. Martín-Sacristán and J. F. Monserrat, "Channel Modelling based on Game Engines Light Physics for $\mathrm{mmW}$ in Indoor Scenarios," Submmitted to European Conference on Antennas and Propagation 2020 (EuCAP 2020), Mar. 2020, pp. 1-5.

[C2] D. Prado, S. Inca, D. Martín-Sacristán and J. F. Monserrat, "Comparison of Optimization Methods for Aerial Base Station Placement with Users Mobility," in European Conference on Networks and Communications 2019 (EuCNC 2019), Jun. 2019, pp. 1-5.

[C3] C. Herranz, D. Martín-Sacristán, J. F. Monserrat, N. Cardona and S. Inca, "On the Use of Serious Game Engineering for 5G System Performance Evaluation," in European Conference on Networks and Communications METIS-II Special Session WP7 (EuCNC 2016), Jun. 2017, pp. $1-5$.

\section{Related Work}

[R1] A. Bustamante, S. Inca, M. Chuchon, and R.Adrian, "Design of a V2V communications antenna based on LTE technology and IEEE802.11p standard," in 2017 IEEE XXIV International Conference on Electronics, Electrical Engineering and Computing (INTERCON), Apr. 2017, pp. $1-4$. 


\section{Chapter 2}

\section{Evaluation of Path Loss Channel Models for 5G}

This chapter presents an evaluation of path loss channel models for Fifth Generation $(5 \mathrm{G})$ systems and is based on the results obtained in the publication [71]. The aim is to compare the different propagation loss models available with measurement campaigns conducted in urban environments, in order to establish the importance of using map-based models. To this end, firstly, the stochastic channel models and the map-based model of Mobile and wireless communications Enablers for Twenty-twenty Information Society (METIS) are evaluated with a measurement campaign conducted in an Urban Macro-cell (UMa) environment in the frequency bands below $6 \mathrm{GHz}$. And secondly, it evaluates the stochastic and map-based channel models in the bands above $6 \mathrm{GHz}$ by comparison with a measurement campaign in an urban environment in the 28 and $73 \mathrm{GHz}$ bands.

In that sense, this chapter has been divided into the following sections:

- Section 2.1 presents a brief introduction.

- Section 2.2 describes the stochastic channel model of International Mobile Telecommunications 2020 (IMT-2020) and Third Generation Partnership Project (3GPP) and presents its main features.

- Section 2.3 describes the stochastic channel model of New York Univeristy (NYU) and presents its main features.

- Section 2.4 presents a semi-deterministic map-based model proposed by METIS. 
- Section 2.5 evaluates channel propagation models below $6 \mathrm{GHz}$ with a measurement campaign conducted in an urban scenario in Valencia, Spain.

- Section 2.6 evaluates channel propagation models above $6 \mathrm{GHz}$ with a measurement campaign conducted by NYU in an urban scenario on the NYU's Manhattan Campus in the 28, 38 and $73 \mathrm{GHz}$ bands.

- Section 2.7 presents the conclusions of this chapter.

\section{$2.1 \quad$ Introduction}

Studies conducted by the 3GPP have demonstrated the need to establish geometric channel models for more realistic characterization. In that sense, one contribution of METIS project is to develop non-synthetic channel models based on extensive simulations. The purpose of METIS is to establish a mapbased channel model focused on twelve test cases described in [72]. In fact, in the evaluation of candidates for the $5 \mathrm{G}$ technology component, channel models are of crucial importance to ensure accurate modeling of propagation conditions. The International Telecommunications Union (ITU) Radiocommunications Sector (ITU-R) defined in [73] a channel model that can be parameterized to cover a different set of test environments, ranging from indoor to rural. All these models have been incorporating a $3 \mathrm{D}$ characterization of the scenario layout, which is essential for the future evaluation of cellular systems [74]. Models need to take into account the geometry of the scenario and include the accurate location and dimensions of streets and buildings to predict changes in radio propagation $[71]$.

\subsection{IMT-2020 and 3GPP Large-Scale Channel Model}

ITU-R and 3GPP channel models for $5 \mathrm{G}$ are based on the channel models defined by these entities for frequencies below $6 \mathrm{GHz}$, such as the 3GPP-3D and IMT-Advanced models. The update of these models has extended the frequency range from $0.5 \mathrm{GHz}$ to $100 \mathrm{GHz}$. These models are applicable within a set of test environments such as Indoor Hotspot (InH), UMa, Urban Microcell (UMi) y Rural Macro-cell (RMa) 


\subsubsection{Large-Scale Model}

The equations for each of the scenarios are available in [75]. For the path loss estimation in Line of Sight (LoS) and Non Line of Sight (NLoS), the distances $d_{2 D}$ and $d_{3 D}$ are defined within a 3D model shown in Figure 2.1.

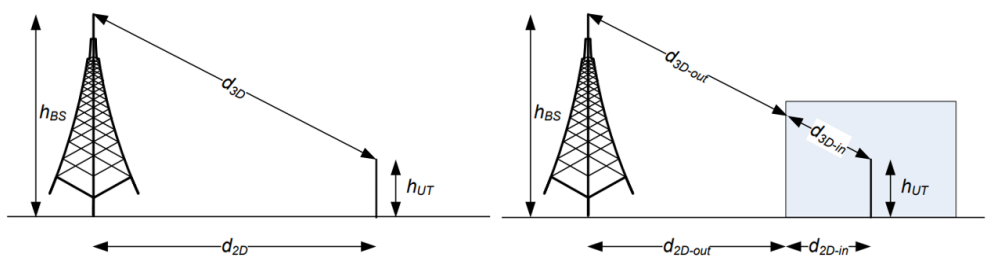

Figure 2.1: Definition of 2D and 3D distances for outdoor environments (left), and for indoor environments (right) [39]

The propagation loss equations depend on the LoS and NLoS conditions and the breakpoint, $d_{B P}^{\prime}$ (see (2.1)). When the distance between Base Station (BS) and User Equipment (UE) is less than $d_{B P}^{\prime}$ the losses are calculated as a function of distance and frequency. And, when the distance exceeds $d_{B P}^{\prime}$, the equations add new terms involving the heights of the BS and UE. After the propagation loss has been estimated, a normally distributed random fading with an average of 0 and a standard deviation of $\sigma_{S F}$ should be added.

$$
\begin{gathered}
d_{B P}=4 h_{B S}^{\prime} h_{U T}^{\prime} \frac{f_{c}}{c}, \\
h_{B S}^{\prime}=h_{B S}-1, \\
h_{U T}^{\prime}=h_{U T}-1 .
\end{gathered}
$$

\subsubsection{Small-Scale Model}

Small-scale parameters specify the characteristics of each of the propagation paths. The model is used to generate and estimate the power and delays of the paths, the Angle of Departures (AoDs) and the Angle of Arrivals (AoAs). In order to calculate these small-scale parameters, the procedure is defined in [39]. This procedure can be summarized in the following steps: first the delays are generated with an exponential distribution. Second, a power cluster is generated assuming an exponential power delay profile with a single slope. Third, the AoDs and AoAs are generated in both azimuth and elevation following a Gaussian distribution. Fourth, the rays are randomly matched within a group for both azimuth and elevation angles. Fifth, cross-polarization power ratios (normally distributed) are generated for each beam for each cluster [39]. 


\subsubsection{LoS Probability}

The LoS probability of ITU-R and 3GPP is a function that depends on the heights and separation between transmitter and receiver and are available for several scenarios, available in [75]. Probability functions are derived from models for bands below $6 \mathrm{GHz}$ of $3 \mathrm{GPP}$. In (2.4), the probability function for a UMa scenario is shown. It can be indicated that the LoS probability for distances less than 18 meters is 1 , and for larger distances, LoS probability is inversely related to the distance $d_{2 D}$ and directly related to the height of the user when it is longer than 13 meters (variable $C^{\prime}\left(h_{U T}\right)$ ).

$$
P_{L O S}= \begin{cases}1 & d_{2 D} \leq 18 m \\ {\left[\frac{18}{d_{2 D}}+\exp \left(-\frac{d_{2 D}}{63}\right)\left(1-\frac{18}{d_{2 D}}\right)\right](1+} & \\ C^{\prime}\left(h_{U T}\right) \frac{5}{4}\left({\left.\frac{d_{2 D}}{100}\right)}^{3} \exp \left(-\frac{d_{2 D}}{150}\right)\right) & 18<d_{2 D}\end{cases}
$$

where,

$$
C^{\prime}\left(h_{U T}\right)= \begin{cases}0 & h_{U T} \leq 13 m \\ \left(\frac{h_{U T}-13}{10}\right) & 13<h_{U T} \leq 23 m\end{cases}
$$

\subsection{NYU Propagation Channel Model}

The NYU propagation loss model [40] is an extension of the channel propagation models proposed by the $3 \mathrm{GPP}$ with minor modifications. The NYU model is valid for frequencies in the range of 6 to $100 \mathrm{GHz}$. Its large potential is based on the extensive measurement campaigns carried out in the frequency range from 2 to $73 \mathrm{GHz}$ in UMa, UMi, InH scenarios. The advantage of NYU extensive measurement campaigns is that it allows for a propagation loss model that fits the evaluation scenario. In fact, NYU Propagation Channel Model includes: LoS, NLoS and blockage modeling with a squared exponential term, wideband power delay profiles, time clusters and spatial lobe, physics-based path loss model. The latter model is using the existing 3GPP path loss equations, providing a standard and stable definition of "path loss exponent" across all different parties, scenarios, and frequencies [75].

\subsubsection{Large-Scale Model}

For the large-scale propagation loss estimation it proposes an equation for both LoS and NLoS, as shown in (2.6). Where $d_{0}$ is the reference distance, $n$ is the Path Loss Exponent (PLE), an optimization factor that depends on the 
scenario where the quadratic mean error has a better fit. $X_{\sigma}^{C I}$ is the scattering factor that represents large-scale signal fluctuations as a result of shadows due to wireless channel obstructions and is defined as a zero-mean Gaussian random variable with standard deviation $\sigma$ in $\mathrm{dB}[76]$. For UMi and UMa scenarios, the values of the PLE for UMi are 2 and 3.2 for LoS and NLoS respectively and 2 and 2.9 for UMa for LoS and LoS respectively [77].

$$
P L(d)[d B]=P L\left(d_{0}\right)+10 n \log _{10}\left(\frac{d}{d_{0}}\right)+X_{\sigma}^{C I},
$$

where $P L\left(d_{0}\right)$ is the loss in free space in $d B$ defined as:

$$
P L\left(d_{0}\right)[d B]=10 \log _{10}\left(\frac{4 \pi d_{0}}{\lambda}\right)^{2} .
$$

\subsubsection{Small-Scale Model}

In NYU, small-scale parameters includes time cluster excess delays, time cluster powers, and mean AoD and AoA azimuth and elevation angles for each spatial lobe [40]. For UMi and UMa scenarios, the number of time clusters, the number of subpaths per time cluster, and the number of spatial lobes do not hold particular values but follow certain distributions and can vary in each channel realization. Furthermore, the numbers of clusters in the 3GPP model are much higher as compared to the measured numbers of time clusters (around 3 to 4 on average)and spatial lobes (about 2 on average) in NYU [40].

\subsubsection{LoS Probability}

The NYU model was developed using a finer resolution intersection test than 3GPP [78]. This function was developed based on extensive measurement campaigns conducted mainly in New York City [38]. The probability function of the NYU model is similar to 3GPP [37] but with the second part of the equation squared and with a cutting distance for $d_{2 D}$ equal to 20 meters, see (2.8). The evaluation of this quadratic function shows an improvement in its model accuracy by including a square throughout the term [38, 79].

$$
P_{L O S}= \begin{cases}1 & d_{2 D} \leq 20 m \\ {\left[\frac{18}{d_{2 D}}+\exp \left(-\frac{d_{2 D}}{63}\right)\left(1-\frac{18}{d_{2 D}}\right)\right](1+} & \\ C^{\prime}\left(h_{U T}\right) \frac{5}{4}\left({\left.\frac{d_{2 D}}{100}\right)}^{3} \exp \left(-\frac{d_{2 D}}{150}\right)\right)^{2} & 20<d_{2 D} .\end{cases}
$$




\subsection{METIS Map-Based Channel Model}

In contrast to stochastic models, map-based models have identified the necessity to develop propagation models that better fit with real scenarios. The model uses ray-tracing techniques to obtain large-scale fading characteristics for a site-specific environment, and measurement-based results to model the small-scale fading statistics [43]. To explain the map-based model proposed by METIS, an urban scenario is considered, where the base station is located on the roof of a building and has a dominant visibility of users. For the urban macrocells scenario, most of the signal reaches users through diffraction and the main propagation path is over buildings [80]. This propagation scenario is similar to the scenario assumed by European Telecommunications Standards Institute (ETSI) in [81]. To generate channel samples between a transmitter and a receiver, the mobility and exact location of both ends must be known. Based on this information, all large-scale parameters are generated, followed by small-scale parameters. Figure 2.2 illustrates the used geometry and the set of variables that modify the model response.

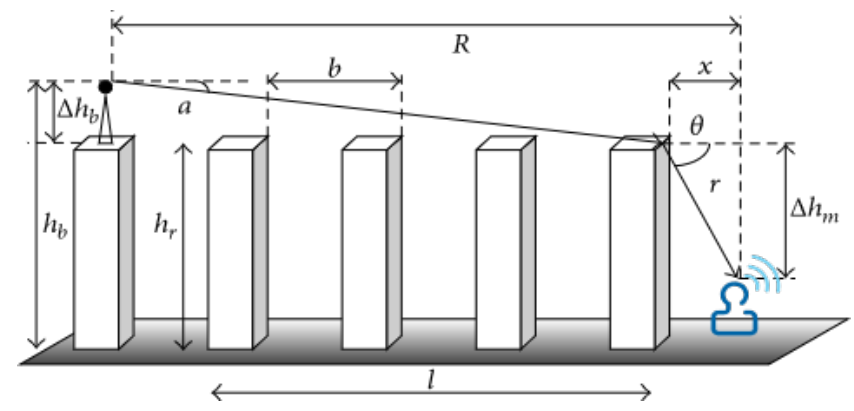

Figure 2.2: Geometry of the large scale model [71]

\subsubsection{Large-Scale Model}

Compared to ray-tracing, the proposed model is equivalent to single-raytracing. The total propagation loss in decibels is expressed as the sum of the loss of free space, the loss of diffraction from the roof to the street, and the fading due to the diffraction of multiple edges beyond the rows of buildings.

$$
L(R)= \begin{cases}L_{f s}+L_{r t s}+L_{m d s} & \text { if } L_{r t s}+L_{m s d}>0 \\ L_{f s} & \text { if } L_{r t s}+L_{m s d} \leq 0\end{cases}
$$


Given a separation between the base station and the mobile device $R$, the free space loss between them is given by:

$$
L_{f s}=-10 \cdot \log _{10}\left(\frac{\lambda}{(4 \pi R}\right)^{2} .
$$

The diffraction from the rooftop to the street is calculated as

$$
L_{r t s}=-20 \cdot \log _{10}\left[\frac{1}{2}-\frac{1}{\pi} \arctan \left(\operatorname{sign}(\theta) \sqrt{\frac{\pi^{3}}{4 \lambda} r(1-\cos \theta}\right)\right],
$$

where

$$
\begin{gathered}
\theta=\tan ^{-1}\left(\frac{\triangle h_{m}}{x}\right), \\
r=\sqrt{\triangle h_{m}^{2}+x^{2}}
\end{gathered}
$$

and where $\triangle h_{m}$ is the difference between the height of the last building and the height of the mobile station antenna and $x$, is the horizontal distance between the mobile and the diffraction edge.

The diffraction loss due to propagation through rows of buildings depends on the height of the base antennas in relation to the heights of the building and on the angle of incidence [82]. This distance, $d_{s}$, is defined as,

$$
d_{s}=\frac{\lambda R^{2}}{\triangle h_{b}^{2}}
$$

where $\triangle h_{b}$ is the height of the base station above roof-top. To calculate $L_{m s d}$, $d_{s}$ is compared to the distance of the road covered by the buildings " $l$ ". If $l>d_{s}$,

$$
L_{m d s}=L_{b s h}+k_{a}+k_{d} \log _{10} \frac{R}{1000}+k_{f} \log _{10}(f)-9 \log _{10}(b),
$$

where

$$
L_{b s h}= \begin{cases}-18 \log _{10}\left(1+\triangle h_{b}\right) & \text { para } h_{b}>h_{r} \\ 0 & \text { para } h_{b} \leq h_{r}\end{cases}
$$

$L_{b s h}$ is the loss that depends on the height of the base station.

$$
k_{a}= \begin{cases}54 & \text { para } h_{b}>h_{r} \\ 54-0.8 \triangle h_{b} & \text { para } h_{b} \leq h_{r}, R \geq 500 \\ 54-1.6 \triangle h_{b} \frac{R}{1000} & \text { para } h_{b} \leq h_{r}, R<500,\end{cases}
$$




$$
k_{d}= \begin{cases}18 & \text { para } h_{b}>h_{r} \\ 18-15 \frac{\triangle h_{b}}{h_{r}} & \text { para } h_{b} \leq h_{r}\end{cases}
$$

and $k_{f}=0.7(f / 925-1)$ for medium-sized cities and suburban centers with medium tree density, whereas $k_{f}=15(f / 925-1)$ for metropolitan environments. Note that the frequency is expressed in $\mathrm{MHz}$.

On the other hand, if $l \leq d_{s}$, the following expression should be considered for the calculation of $L_{m s d}$ :

$$
L_{m d s}=-10 \cdot \log _{10}\left(Q_{M}^{2}\right),
$$

where

$$
\begin{gathered}
Q_{M}= \begin{cases}2.35\left(\frac{\triangle h_{b}}{R} \sqrt{\frac{b}{\lambda}}\right)^{0.9} & \text { para } h_{b}>h_{r} \\
\frac{b}{R} & \text { para } h_{b} \simeq h_{r} \\
\frac{b}{2 \pi R} \sqrt{\frac{\lambda}{\rho}}\left(\frac{1}{\vartheta}-\frac{1}{2 \pi+\vartheta}\right) & \text { para } h_{b} \leq h_{r},\end{cases} \\
\vartheta=\tan ^{-1}\left(\frac{\triangle h_{b}}{b}\right), \\
\rho=\sqrt{\triangle h_{b}^{2}+b^{2}} .
\end{gathered}
$$

Finally, the minimum coupling loss is set to $70 \mathrm{~dB}$.

\subsubsection{Small-Scale Model}

The small-scale statistics are generated following the 3GPP modeling approaches, described in [75].

\subsection{Evaluation of Large-Scale Path Loss Chan- nel Models Below $6 \mathrm{GHz}$}

\subsubsection{Measurement Campaign}

The comparison between the three alternatives, IMT-2020, 3GPP and METIS model was made using real measurements. The measurement campaign was carried out in an urban area of the city of Valencia, Spain. This scenario was chosen because of its heterogeneity, far from the classic regular Manhattan grid assumed in other scenarios. It is a scenario with an irregular pattern of buildings of different heights, sidewalks, parking lots and vegetation. A Universal Mobile Telecommunications System (UMTS) base station operating on the 
$2700 \mathrm{MHz}$ frequency was chosen for the evaluation of the models. Figure 2.3 shows the area where the measurements were taken and the location of the transmitting antenna. The scenario corresponds to a UMa scenario, where the transmitter is located above the height of the surrounding buildings at a height of 37 meters.

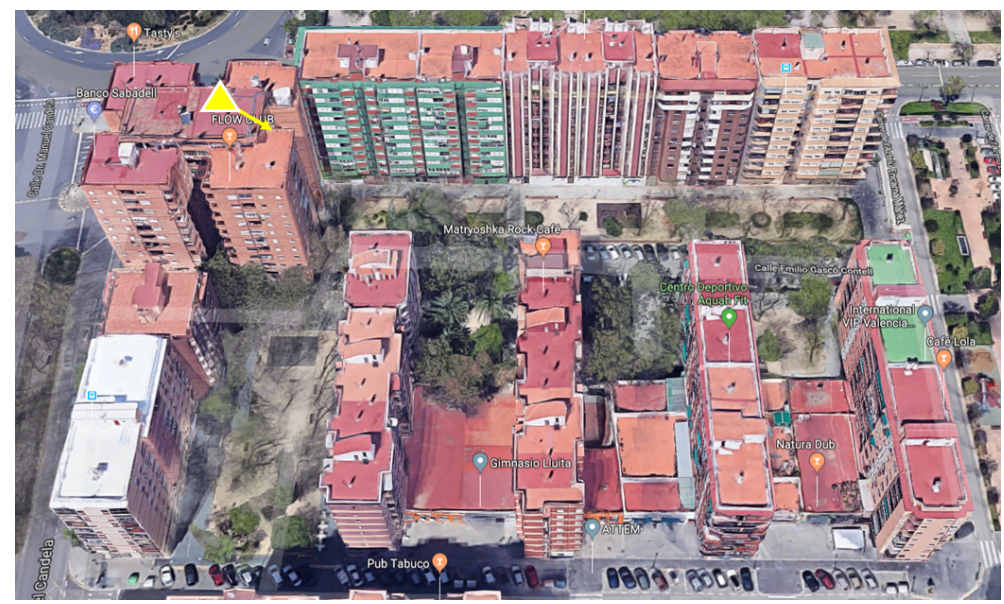

Figure 2.3: Map of the site in Valencia, including the location and azimuth of the cell under study

The indicator used is the Receive Signal Code Power (RSCP). Given that the RSCP measurement of the pilot channel (CPICH) takes as reference the antenna connector, it is considered a good indicator to estimate the path losses once the transmit power, mobile terminal antenna gain and base station antenna gains are known. RSCP measurements were obtained using a terminal equipped with Nemo-Outdoor software. The scenario was divided into areas of squares of side 10 meters and the measurements were taken in the central position of each grid at a height of $1.5 \mathrm{~m}$. The measurements were taken simulating static measurements where 60 samples were stored for 30 consecutive seconds. The measurements taken were 1000 times the coherence time of a Rayleigh-Fading fading channel corresponding to a low-mobility user or pedestrian traveling at $3 \mathrm{~km} / \mathrm{h}$, so that the average of the samples can be considered independent of the fast fading [71].

The approximate values of propagation loss were obtained from the RSCP levels, considering the base station transmit power, mobile terminal gain and base station antenna gain for each point, calculating the approximate values for azimuth and elevation and the exact antenna pattern. To create the scenario 
heights map, a matrix was created with the exact heights from the ground. The Figure 2.4 shows the 3D map used to estimate the losses with a resolution of $10 \mathrm{~m}$.

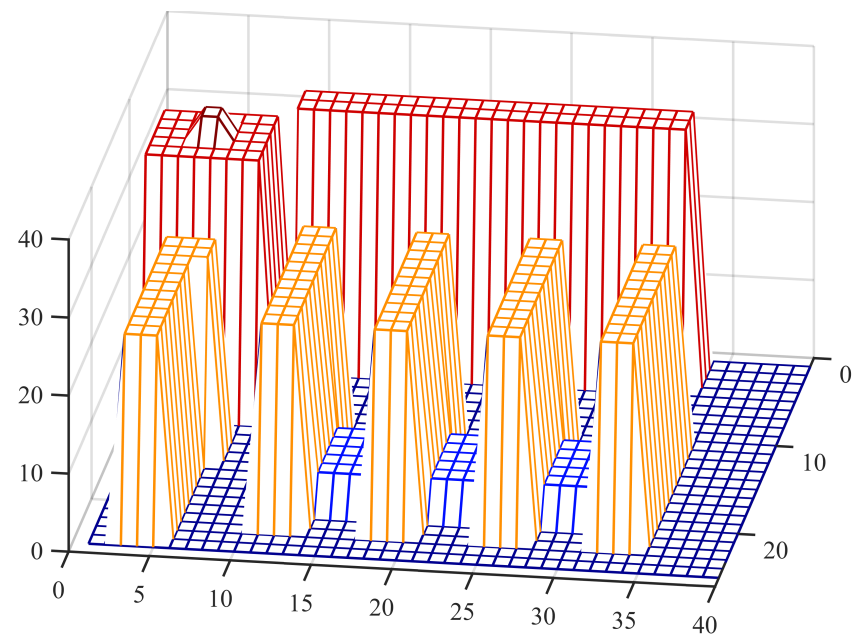

Figure 2.4: Map model used for the channel prediction

The height map is used to calculate the exact values of the variables shown in the Figure 2.2 and, also, to determine the LoS or NLoS conditions for IMT-2020 and 3GPP models. Propagation losses were estimated under these conditions in order to eliminate the error of the LoS probability functions of IMT-2020 and $3 \mathrm{GPP}$.

\subsubsection{Preliminary Results}

Figure 2.5 shows a Cumulative Distribution Function (CDF) of the absolute error of the models IMT-2020, 3GPP and METIS with respect to the real measurements. A strong similarity can be observed between the models of IMT-2020 and 3GPP. With respect to the map-based model of METIS, it can be noted that the calculated propagation loss measures are pessimistic. However, the values are closer to the measured values.

It is worth mentioning that for LoS, i.e. for the lowest range of path loss values, the map-based model is very similar to the 3GPP and IMT-2020 models but differs considerably for higher values, which correspond to NLoS. The values obtained for LoS with the three models are below the measured values, this difference is probably due to the presence of trees that are not included in 
the calculations. On the other hand, the values obtained for NLoS, the error is obtained in $\mathrm{dB}$ as the difference between the measurement and the prediction, and the Root Mean Square Error (RMSE)indicator is the Root Mean Square of that error. The RMSE between the map-based model and the measured values is $7.21 \mathrm{~dB}$. And, IMT-2020 and 3GPP have an RMSE of $15.85 \mathrm{~dB}$ and $14.75 \mathrm{~dB}$ respectively. It can be indicated that, due the measurement scenario is not a homogeneous scenario such as those typically used for calibration models of IMT-2020 and 3GPP, such as the Manhattan grid, the error under NLoS conditions is significant.

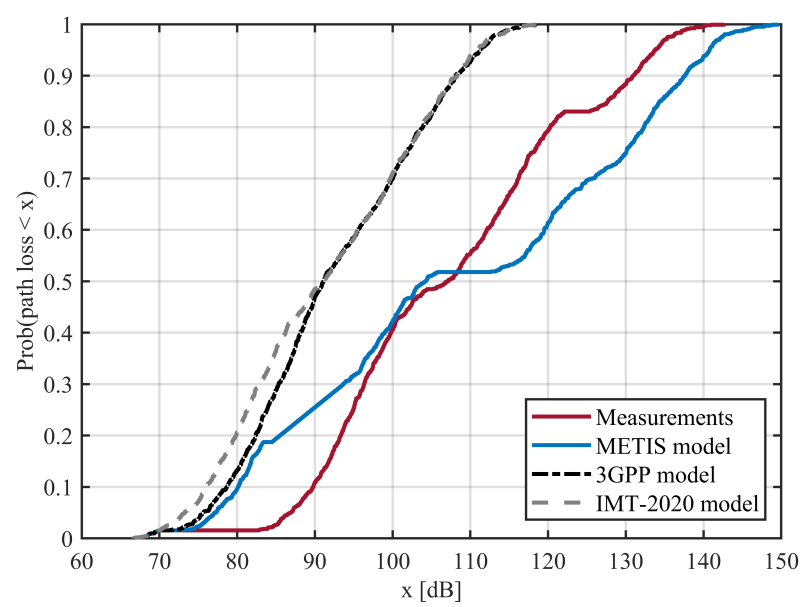

Figure 2.5: CDF of the path loss values in the area under study

Figure 2.6 shows the Probability Distribution Function (PDF) of the absolute error of the predictions for the three models under study. Again, the map-based model features lower error as compared with the other two models. In fact, the error of the map-based model as compared with the real measurements is always lower than $18 \mathrm{~dB}$.

\subsection{Evaluation of Large Scale Path Loss Chan- nel Models Above $6 \mathrm{GHz}$}

\subsection{1 $28 \mathrm{GHz}$ Measurement Campaign}

The measurement campaign was carried out by the group led by Rappaport from the NYU. For the $28 \mathrm{GHz}$ band, measurements were made at the main 


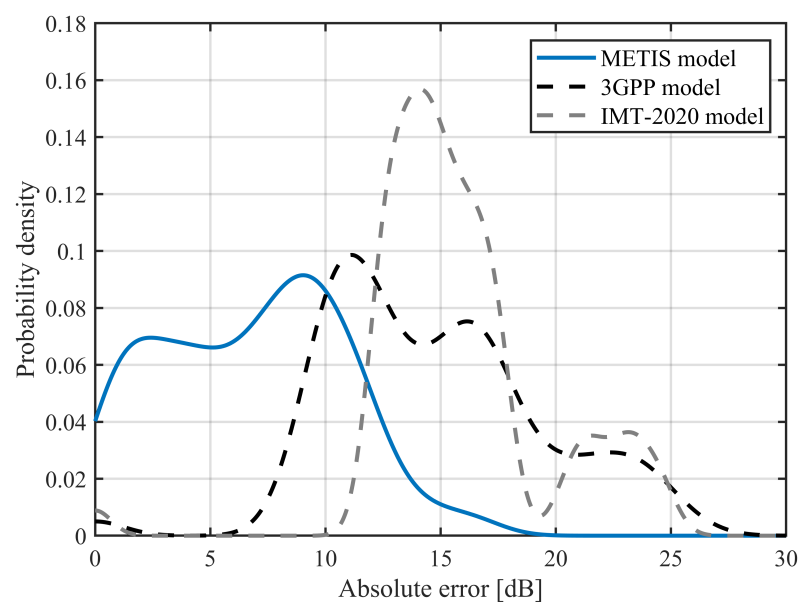

Figure 2.6: Probability distribution of the absolute error in the path loss estimation

campus of the NYU in Manhattan [24, 49, 83, 84]. Figure 2.7 shows the scenario that contains buildings with non-uniform geometries and different heights, main and secondary streets, parks, foliage, among others, typical of a real dense urban scenario. Measurements were made at 3 TX locations and 27 RX locations, considering communications LoS and NLoS. Narrow-beam antennas were used for TX and RX, with gain of 24.5 dBi and Half-Power Beamwidth (HPBW) of $10.6^{\circ}$ and $8.6^{\circ}$ in azimuth and elevation respectively and $\mathrm{RF}$ transmit power of $30.1 \mathrm{dBm}$ [40]. The locations (geographic coordinates) and heights of the TX and RX are available in [49]. The TX - RX separation distances range from 31 to 425 meters. The heights used for the TX were 7 and 17 meters, located on roofs above the streets and with tall buildings around them. A typical RX height of 1.5 meters was used. Each measurement between TX-RX, the RX antenna made sweeps in both azimuth and elevation to acquire the Power Delay Profile (PDP) in each of the orientation increments. The values obtained for each TX-RX combination correspond to the omnidirectional propagation loss, where the maximum dynamic range achieved for path loss measurements is $178 \mathrm{~dB}$.

\subsection{2 $73 \mathrm{GHz}$ Measurement Campaign}

The measurement campaign was carried out by the group led by Rappaport from the NYU. For the $73 \mathrm{GHz}$ band, measurements were made at the main 


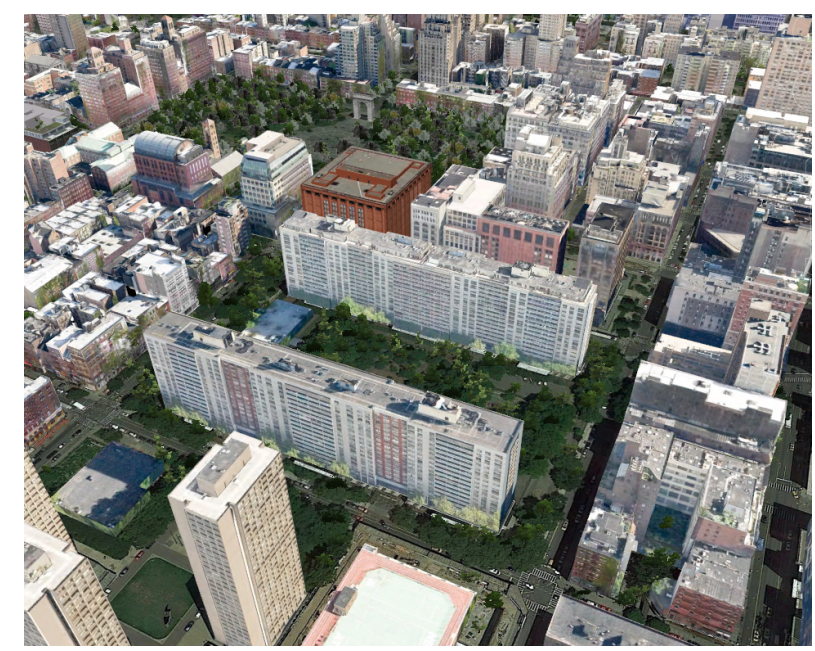

Figure 2.7: Map of TX and RX locations around NYU's Manhattan campus for 28 and $73 \mathrm{GHz}$

campus of the NYU in Manhattan [24, 49, 83, 84]. The scenario is the same as described for the $28 \mathrm{GHz}$ band. Measurements were made at $5 \mathrm{TX}$ locations and 27 RX locations, considering both LoS and NLoS communications. Narrowbeam antennas were used for TX and RX, with gain of $27 \mathrm{dBi}$ and HPBW of $7^{\circ}$ in azimuth and elevation and RF transmit power of $14.6 \mathrm{dBm}$ [40]. The locations (geographic coordinates) and heights of the TX and RX are available in [49]. The TX - RX separation distances range from 27 to 190 meters. The heights used for the TX were four at 7 and one at 17 meters, located on roofs above the streets and with tall buildings around them. The RX height was set at 2 and 4.06 meters. As for $28 \mathrm{GHz}$, for each measurement between TX-RX, the RX antenna made sweeps in both azimuth and elevation to acquire the PDP in each of the orientation increments. The values obtained for each TXRX combination correspond to the omnidirectional propagation loss, where the maximum dynamic range achieved for path loss measurements is $181 \mathrm{~dB}$.

\subsubsection{Preliminary Results}

For the evaluation of the propagation models, a 3D model of the NYU Manhattan campus scenario was developed. Figure 2.8 shows the NYU scenario with a resolution of 10 meters. The locations and heights of the receptors were transformed to their equivalent cartesian coordinates for evaluation. 


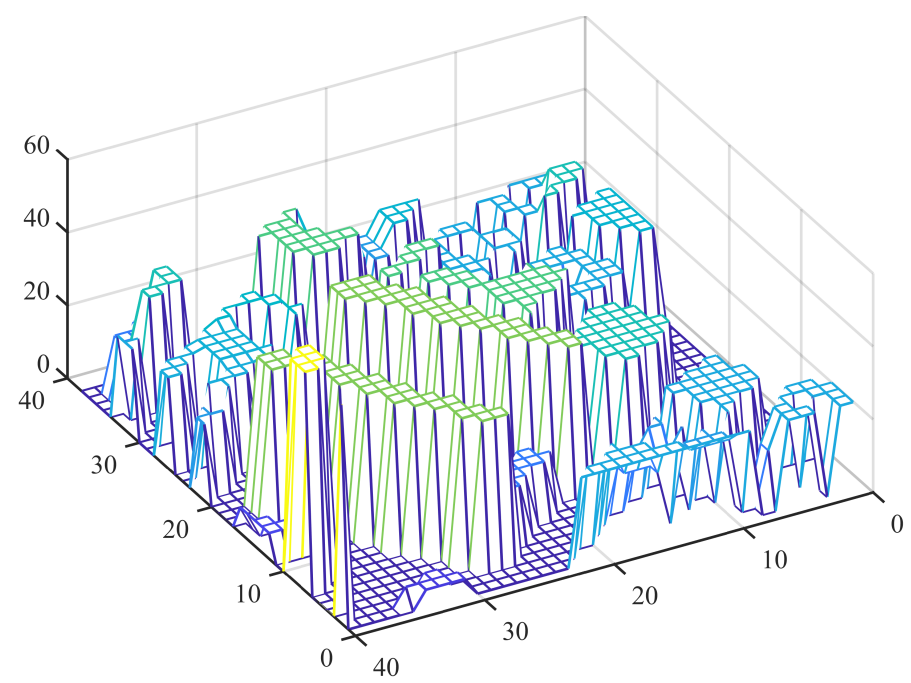

Figure 2.8: NYU's Manhattan campus 3D-Map model used for the channel prediction

For the $28 \mathrm{GHz}$ band, the RMSE obtained with 3GPP, NYU and METIS models are $9.24 \mathrm{~dB}, 8.25 \mathrm{~dB}$ and $7.35 \mathrm{~dB}$ respectively. Figure 2.9 shows the measurements and estimated path loss by the propagation models as a function of distance. It can be noted that, for LoS, the behavior of the three models is similar. In fact, the RMSE obtained for LoS are similar, for $3 \mathrm{GPP}$ and NYU is $6.41 \mathrm{~dB}$ and for METIS is $6.97 \mathrm{~dB}$. For NLoS communications there is a clear difference between the stochastic models (3GPP, NYU) and the map-based model (METIS). It can be observed that the METIS model for NLoS not only considers the separation between the TX and RX, also some other variables corresponding to the geometry of the scenario that allow an adjustment to the measured value. Although the values of RMSE have a difference of less than $2 \mathrm{~dB}$ between the models, we must consider that the difference between the measured and estimated values in some NLoS points for $3 \mathrm{GPP}$ and NYU are up to $25.71 \mathrm{~dB}$ and $23.56 \mathrm{~dB}$ respectively, while for METIS the maximum difference is $10.39 \mathrm{~dB}$.

Figure 2.10 shows the PDF of the absolute error. Similarity is seen between the 3GPP and NYU models, with a slightly better error probability for the NYU model. On the other hand, it is observed that the results obtained with the METIS model show a better consistency and less probability of error. 


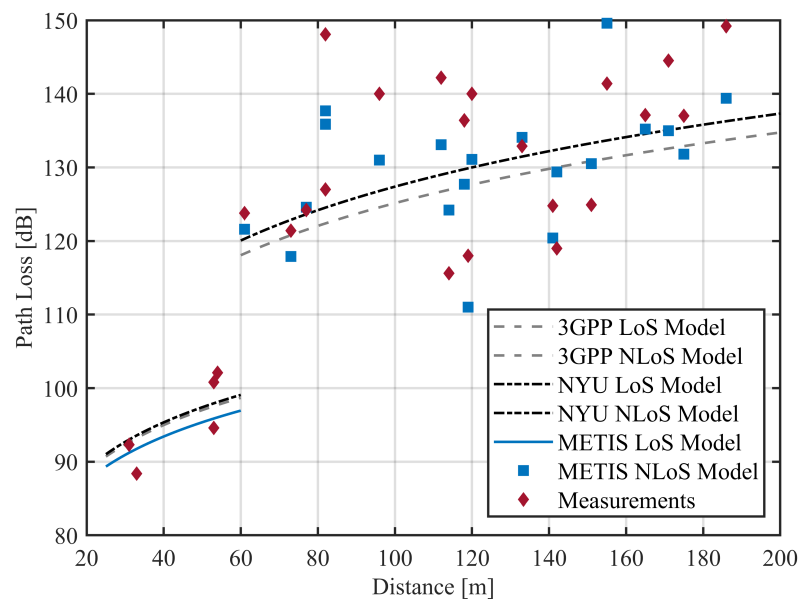

Figure 2.9: Path loss correlation for $28 \mathrm{GHz}$

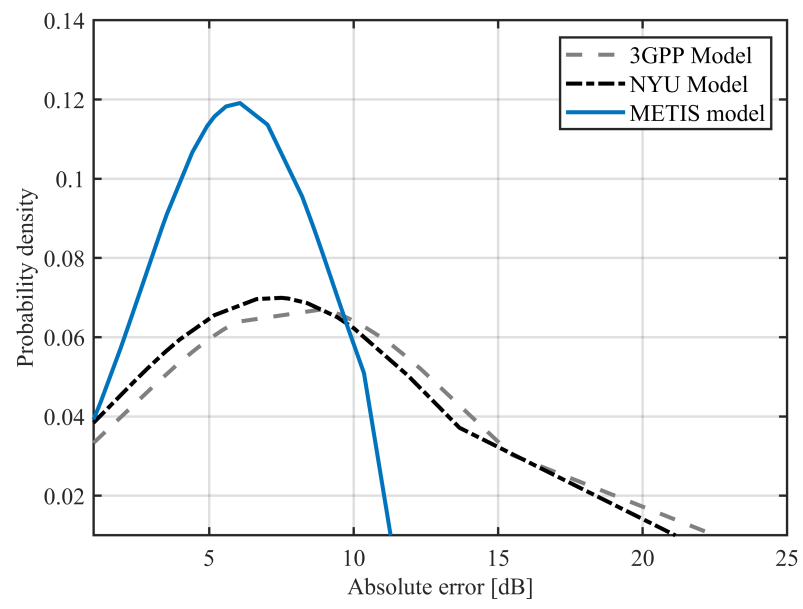

Figure 2.10: Probability distribution of the absolute error in the path loss estimation for $28 \mathrm{GHz}$

Figure 2.11 shows the measurements and estimated path loss by the propagation models as a function of distance at $73 \mathrm{GHz}$. The RMSE obtained with 3GPP, NYU and METIS models are $9.59 \mathrm{~dB}$ and $9.41 \mathrm{~dB}$ and $8.32 \mathrm{~dB}$ respectively. As for the $28 \mathrm{GHz}$ band, the METIS model gets a lower RMSE. However, for all models, the maximum differences in measured and estimated 
values reach up to $24 \mathrm{~dB}$ at some points. It is considered that the accuracy of the 3D map used for the measurements can degrade the RMSE in METIS model, as well as the presence of vegetation and other elements that are not included.

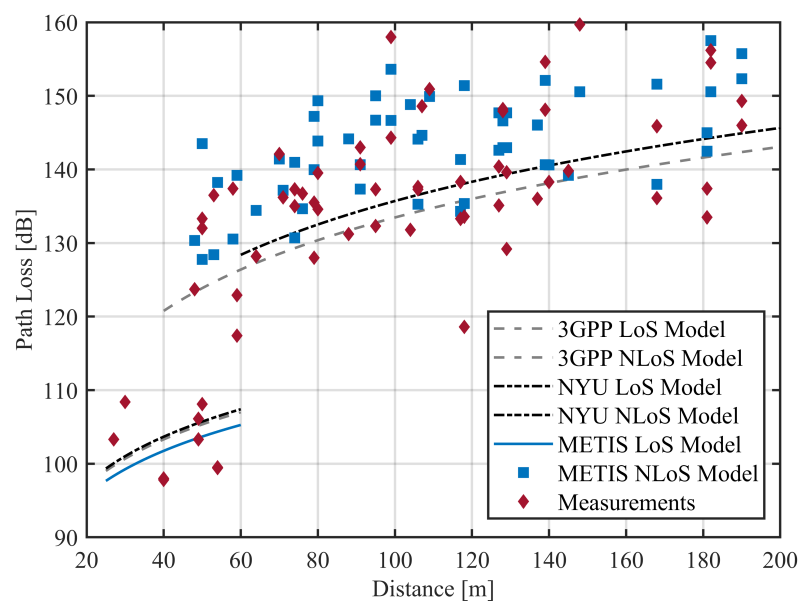

Figure 2.11: Path Loss correlation for $73 \mathrm{GHz}$

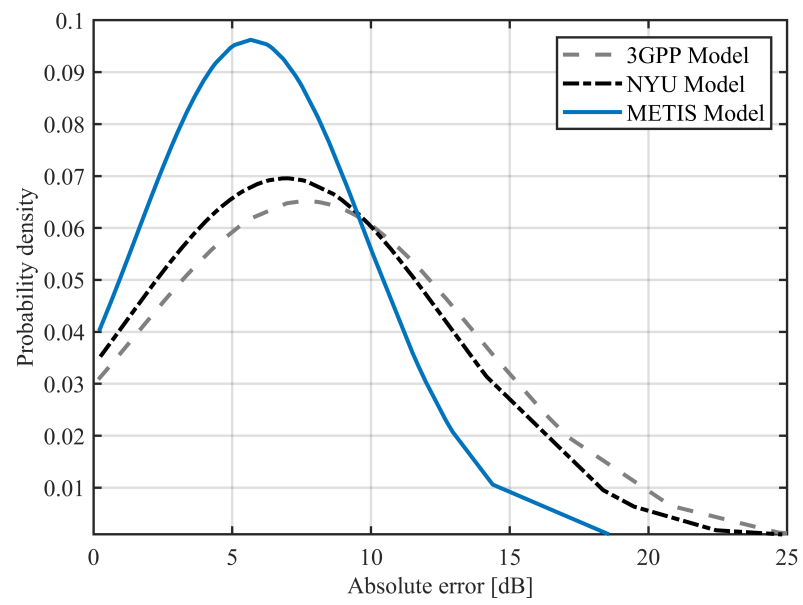

Figure 2.12: Probability distribution of the absolute error in the path loss estimation for $73 \mathrm{GHz}$ 
Figure 2.12 shows the PDF of the path loss predictions. As in the $28 \mathrm{GHz}$ measurements similarity, is seen between the 3GPP and NYU models, with a slightly better error probability for the NYU model.

\subsection{Conclusions}

This chapter presents the evaluation of large-scale propagation channel models for bands below $6 \mathrm{GHz}$. This work was published in [71] as results of the investigation of this Thesis. It also presents an extension of the evaluation for bands above $6 \mathrm{GHz}$, on the $28 \mathrm{GHz}$ and $73 \mathrm{GHz}$ bands in a urban scenarios. In general, it is shown that the METIS model has a better performance as compared with the IMT-2020, 3GPP and NYU models for all bands studied. The results obtained allow us to state that it is necessary to have a model that considers the geometry of the scenario in order to adequately estimate the behavior of radio propagation. This holds especially for frequencies above $6 \mathrm{GHz}$, where radio propagation is more vulnerable to blocking objects and phenomena associated with propagation. 



\section{Chapter 3}

\section{Unity3D Game Engine}

This chapter presents a description of the Unity game engine. Mainly, it focuses on the analysis and description of the light engine and the ray-tracing algorithms used for lighting and rendering, which are tools used for the acquisition of light intensity for the Light Intensity Model (LIM) proposed in this Thesis.

To that end, this chapter has been divided into the following sections:

- Section 3.1 presents a global description of the Unity game engine.

- Section 3.2 describes Unity's interface.

- Section 3.3 presents the main characteristics of the $3 \mathrm{D}$ objects that compose the virtual scenario.

- Section 3.4 explains the lighting techniques available at Unity, both direct and indirect lighting.

- Section 3.5 presents the main characteristics of the different light sources available in Unity.

- Section 3.6 describes Unity's light engine and explains the ray-tracing algorithms used in the lighting process.

- Section 3.7 presents the material properties of Unity objects, including textures and shaders.

- Section 3.8 draws the main conclusions of the chapter. 


\subsection{Unity Game Engine Description}

Unity is a platform for the development of videogames created by Unity Technologies. In 2005, the first Unity release was launched; it was focused exclusively on the Mac platform. In 2010, the first multi-platform version was launched, with support for Windows, Linux and Mac, and focused on developing tools for game developers. Unity's goal was to provide a tool that supports interactive content in $2 \mathrm{D}$ and $3 \mathrm{D}$, with an open community, global use and support of the most widely used computer operating systems (Windows, Mac and Linux), mobile operating systems (iOS, Android and Microsoft), consoles (Play Station, Wii, XBox) and major web search engines (Google Chrome, Mozilla, Internet Explorer, Safari, Opera).

There are two ways to install Unity. The first, from the Unity Hub, which allows you to manage and install multiple versions of Unity simultaneously and independently. And, the second, by directly downloading a specific version of the software from the Unity repository available at [85]. One of the advantages of Unity is that it is compatible with many external platforms, which facilitates the development of video games. For example, for object creation and 3D modeling, Unity supports .fbx, .obj, .3dS formats that can be created from external software such as ProBuilder, ScketchUp, 3DS Studio, Maya or similar. Similarly, 3D object meshes are compatible with .3dS, .dfx and .ofb formats and textures with .bmp, .tif, .tga, .jpg and .psd formats. Audio files in .wav and .mp3 formats are also supported.

Unity has a package manager (Unity Package Manager (UPM)) that expands the range of input files it supports. These packages are collections of files and data that help the development of more complex applications. There are a variety of packages available for free or for a fee which can be found at [86]. These packages are solutions that help developers streamline the development process. For example, there are professional city modeling packages or super realistic environments $[87,88]$ that provide highly complex $3 \mathrm{D}$ modeling with a high level of detail. Other packages are oriented to the animation of objects, for example [89, 90], to the simulation of urban traffic or pedestrians, where movement and traffic patterns can be programmed into the scenario. There are also packages that allow the implementation of Artificial Intelligence (AI) and Virtual Reality (VR) algorithms [91, 92].

\subsection{Unity Interface}

Unity's interface is composed of five parts [93] (see Figure 3.1). 
1 Scene View: It is the area where the game is constructed. In this area it is possible to position and manipulate all the objects of the scene.

2 Hierarchy Window: It consists of a list of GameObjects in the scene that could have a hierarchy or parentage.

3 Inspector Window: It is used to view and edit object properties as well as preferences and other settings. The Inspector displays all properties of all components and materials in the object and allows editing. In this window you are able to edit the public variables of the scripts instantiated in the objects without having to open the code editor.

4 Game View: It is the preview of the finished game, which are rendered from the active cameras of the game. It also has settings for screen ratio and screen resolution to facilitate visualization tests.

5 Project Window: The Project window contains all the files organized in folders that are available in the project. It is similar to a library or repository of all the physical components, scripts, textures, among others to be used in the development of the application.

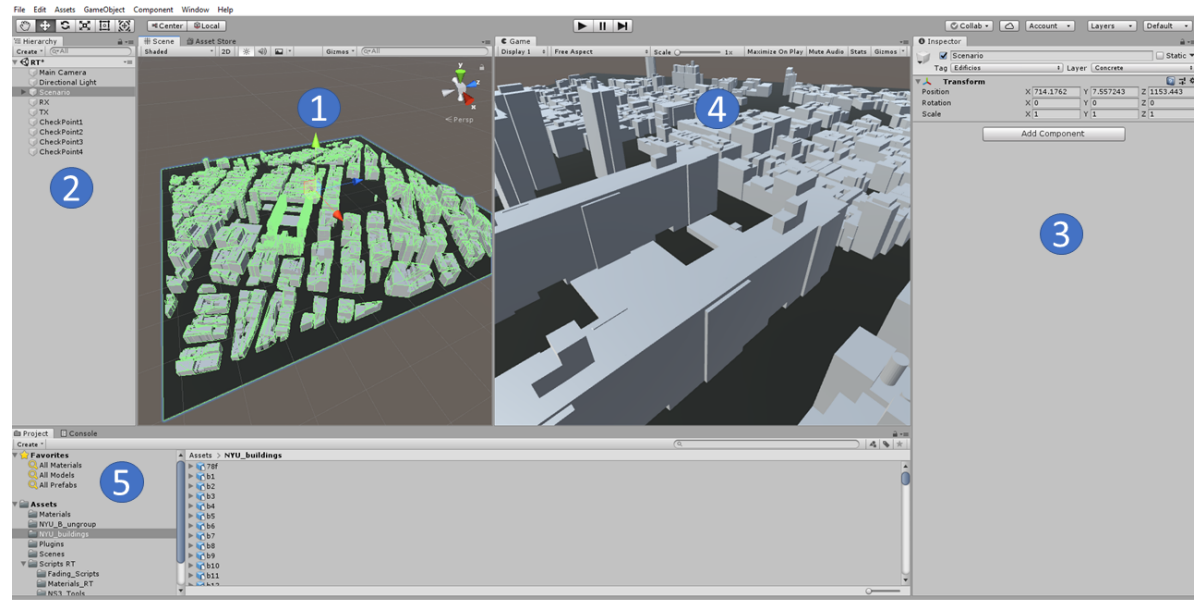

Figure 3.1: Unity interface 


\subsection{Geometry in Unity}

\subsubsection{GameObjects}

Each of the objects that compose the stage in Unity is known as GameObject. GameObjects contain different components according to their functionality. For example, they can be physical objects or collectible objects such as lights and cameras, among others.

Physical objects are composed of a set of interconnected 2D polygons, known as meshes. The 3D primitive objects available in Unity are cube, sphere, capsule and cylinder. However, as discussed in the description of the Unity engine, it is possible to import complex models from a modeling application. All GameObjects have the "Transform" component, which determines the position, rotation, and scale of each object in the scene.

\subsubsection{Meshes}

In Unity, a mesh is a component of solid objects. The mesh is composed of a set of triangles that cover the surface of the object. Figure 3.2 shows two mesh configurations for a building (green lines). On the left, it shows a simple mesh composed of 121 triangles and on the right it shows a complex mesh composed of 7492 triangles. The location and properties of the triangles (vertices, normals, coordinates of textures) are stored in a matrix within the Mesh class.

This component has a direct impact on the performance of lighting and the physical effects. For example, for LIM implementation, it is a requirement for irregular objects to have complex meshes that allow proper calculation of indirect lighting. However, the greater the number of polygons, the more work the Graphics Processing Unit (GPU) must do to render the objects on screen. Similar case, with Ray-Tracing implementation. In this sense, it is necessary to create complex meshes only for irregular and relevant objects.
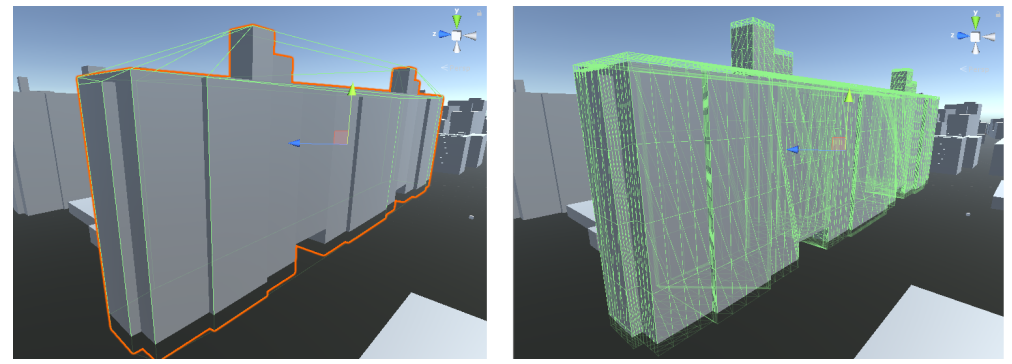

Figure 3.2: Example of building meshes 


\subsection{Lighting Techniques}

In general terms, lighting in Unity can be considered "real time", "baked" or "pre-calculated". All these techniques can be used in combination to create an immersive scene lighting.

\subsubsection{Real-time Lighting}

Real-time lighting is the updating of the lighting between the frames of a scene. In Unity, by default, all light sources are real-time. However, real-time lighting techniques only work for direct lighting and not for indirect lighting. This means that, in order for global lighting to include direct lighting and indirect due to specular and diffuse reflections, it is necessary to use pre-calculated lighting techniques such as Global Illumination (GI).

\subsubsection{Baked Lighting}

Backed Lighting is a process that allows pre-calculating the illumination in the static objects of the scene. As a result of the process we obtain the "light maps", which can include direct and indirect lighting. These results are stored in the textures that are overlaid on the objects of the scene. The purpose of using "light maps" is to reduce the computational cost of the GPU, however it has the following limitations:

- "Light maps" only consider the static objects of the scene.

- "Light maps" cannot change during the game.

- Specular lighting is not implemented in Backed GI.

- Shadows produced by static objects are not updated when light source parameters are changed.

\subsubsection{Pre-Calculated Lighting}

Real-time lighting and baked lighting are not capable of reacting to changes in indirect lighting conditions. pre-calculated Real-time GI offers a technique to update the scene lighting in an interactive way [94]. The concept of this technique lies in calculating indirect lighting using dynamic light maps. This means that Unity updates light maps and calculates indirect lighting using the properties of diffuse lighting [94]. These calculations are performed only in areas close to the user's location, greatly reducing the computational cost of GI. 


\section{CHAPTER 3. UNITY3D GAME ENGINE}

In fact, reducing the light map update area greatly simplifies the number of calculations on the GPU. In fact, by reducing the area where the light map is updated, it greatly simplifies the number of calculations on the GPU. Light maps are also updated when light source properties such as intensity, shadow intensity or even position are changed. The illumination will be updated on an approximation that considers the pre-calculated illumination and the geometry of the scenario. In specific cases, where the aim is to increase the precision of lighting, it is possible to use a combination of these lighting methods. When this is the case, it is necessary to calculate the cost of using the memory of GPU and estimate the computational cost of this process. In section 3.6.1, the GI process is detailed.

\subsection{Light Types}

Light is an electromagnetic radiation produced by the acceleration of a charge [95]. The general properties of light sources are their wavelength and intensity. The light emitted by these sources propagates through the scenario, where it can be reflected, transmitted or scattered. In Unity, four types of light sources are available:

- Directional Lights: This light source simulates the behavior of sunlight and is useful for illuminating very large scenes. The rays emitted by this source are parallel to each other, so the shadows of objects depend on the rotation of the light source. But the intensity of shadows produced by objects does not depend on the distance between the objects and the light source, because the intensity of directional lights does not decrease with distance. On the other hand, the performance of directional light is proportional to the number of pixels it illuminates.

- Point Lights: A point light can be defined as an onmidirectional light source, where the intensity of light is concentrated in the point of origin and decreases proportionally around it as shown in the Figure 3.3. One of the properties of these sources is intensity and range. The intensity refers to the amount of light from the source and the range defines the distance where the light intensity is zero. However, this distance may increase due to reflections and material properties.

As opposed to directional lights, the use of point lights are computationally expensive mainly due to the calculation of shadows and the processing of light reflections. This cost may increase with the use of complex 3D models. 


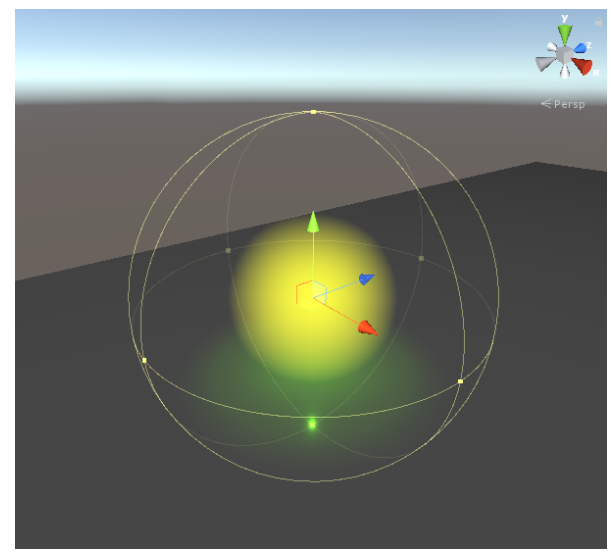

Figure 3.3: Point light

- Spotlights: The spotlights project a cone of light in a defined direction, with a defined intensity and range, as shown in the Figure 3.4. In addition to range and distance, spotlights have an opening angle known as a Spot Angle. The light fade can be seen at the edges of the cone and as a function of distance.

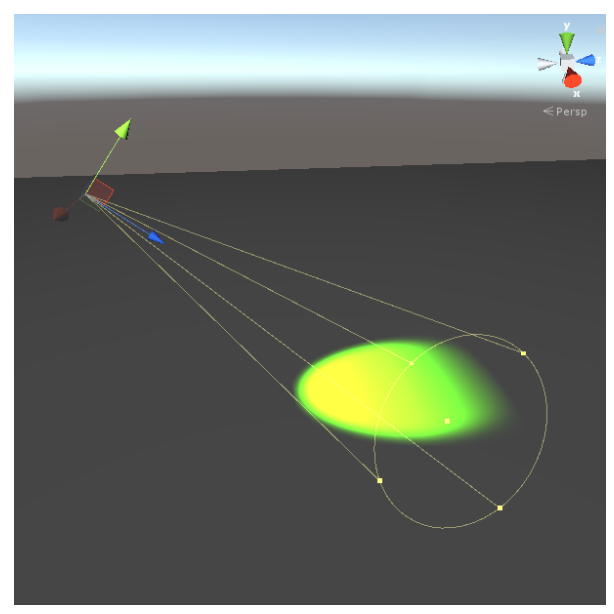

Figure 3.4: Spotlight 
- Area Light: Area lights are defined as rectangles or discs that emit light in all directions from one of their planes (see Figure 3.5). The light intensity decreases in inverse square as it moves away from the source. However, due to its high computational cost it is not available for realtime lighting.

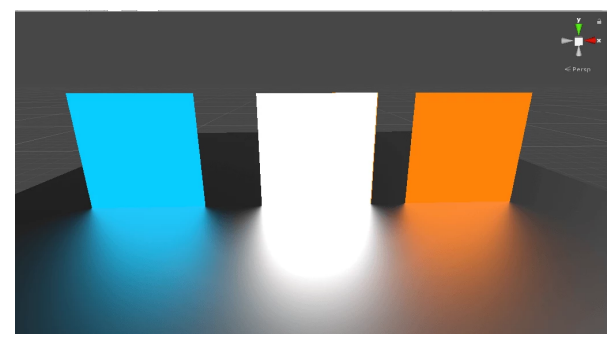

Figure 3.5: Area light

\subsection{Light Physics Engine: Ray-Tracing}

\subsubsection{Global Illumination}

The GI, in computer graphics, is defined as the interaction between light sources and the different objects of a scene [96] [95]. In fact, GI is a group of algorithms and mathematical models that model the behavior of light in scenes. GI tries to simulate the complex behavior of light, reflections, refractions, diffuse reflections and each of the phenomena associated with the propagation of light. The aim of the GI is to create highly realistic scenarios. Unity's graphic engine has implemented a system capable of modeling in real-time the response of direct light as indirect light, with multiple reflections on different surfaces [94].

Implementing GI accurately is computationally expensive and its implementation is a challenge for real-time applications. The most complex case of GI is indirect lighting due to its high computational complexity [97]. Thanks to advances in Central Processing Unit (CPU) and GPU, new strategies have been defined to implement GI in real time. For GI in Unity, indirect lighting is pre-calculated for static objects, which considerably reduces computational cost. This pre-calculation solution in Unity causes a limitation for the indirect illumination computation in moving objects due to a disconnection between static and moving objects in the pre-calculation process. However, the proposed solution to resolve this limitation lies in the use of light probes.

The light probes are based on calculating the indirect illumination in strategic positions of the stage so that in real time the illumination is approximated 


\subsection{Light Physics Engine: Ray-Tracing}

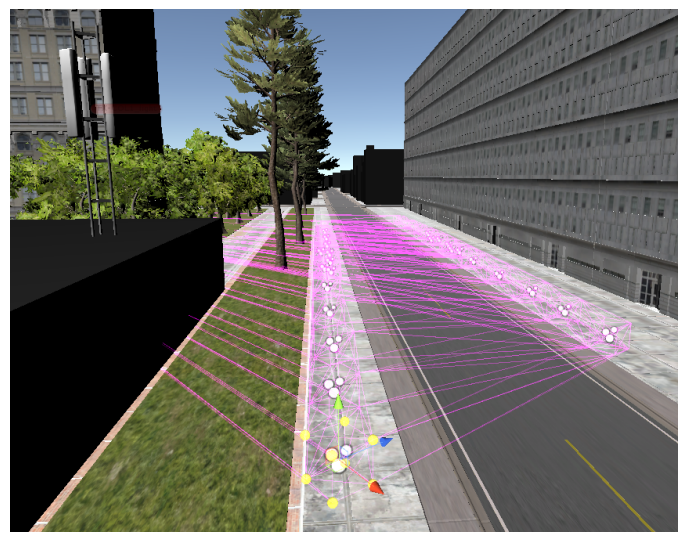

Figure 3.6: Light probes

by interpolating the measurements between the nearest test points and the moving objects. These test points should be strategically placed. For example, in an urban setting, where moving objects are people and vehicles, test points should be placed on sidewalks, as shown in Figure 3.6, for pedestrians and on streets for vehicles.

In general, GI is expressed as the interaction of light with the different components of the scene at a point $p$ (Figure 3.7) with (3.1) [95].

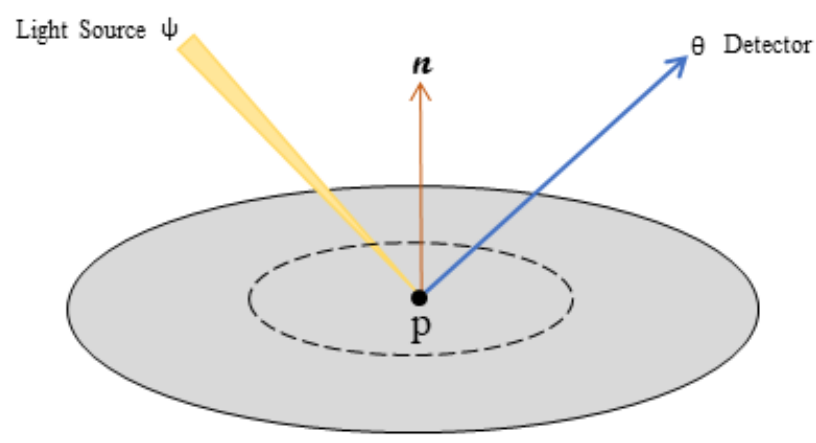

Figure 3.7: BRDF geometry

$$
L(p \rightarrow \theta)=L_{e}(p \rightarrow \theta)+A,
$$


where $L(p \rightarrow \theta)$ is the total radiance at the point $p, L_{e}(p \rightarrow \theta)$ is the radiance emitted at the point $p$ and $A$ is the overall radiance coming from all directions, which is modeled as an integral on the hemisphere of the point $p$.

$$
A=\int_{\Omega_{p}} f_{r}(p, \rightarrow \theta) L_{e}(p \leftarrow \psi) \cos \left(N_{p}, \psi\right) d_{\omega \psi},
$$

where the function $f_{r}$ represents the BRDF, which is a relationship between incident radiation in $\psi$ direction and reflected radiation in $\theta$ direction. $\theta$ is the angle between the normal $n$ and the vector direction of the incident radiation.

\subsubsection{Reflections}

Reflections are one of the main phenomena that are evaluated in Unity's light engines. To understand how this phenomenon is implemented in the GPU, we must first explain the angular dependence of light sources on reflections. As shown in the Figure 3.8 the reflection area $d A$ is proportional to the angle of incidence $\theta$.
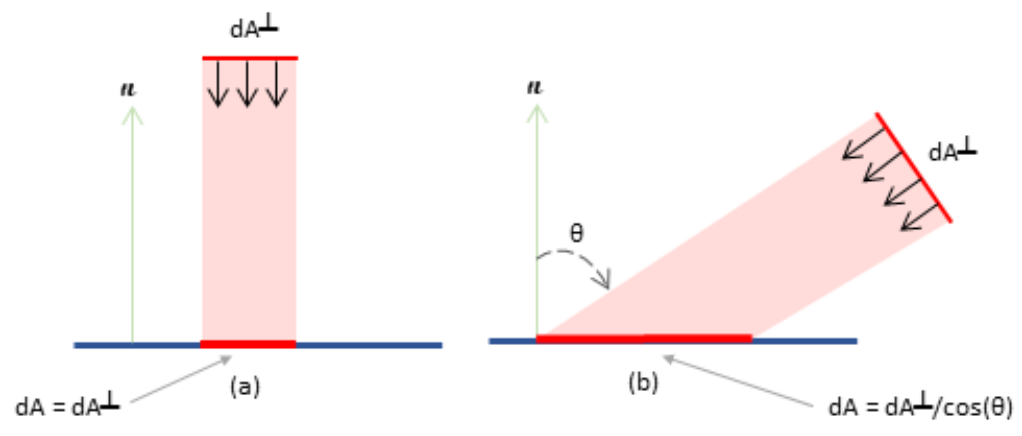

Figure 3.8: Dependence of the reflection on the angle $\theta$

Therefore, radiant light intensity $L$ (reflected) can be defined as

$$
L=\frac{d^{2} \phi}{d A \cos (\theta) d \omega},
$$

where, $\cos \theta$ is the projected solid angle.

In the same way, it is possible to establish a relation between the intensity of the incident light and the irradiated one as

$$
d E i\left(p, \omega_{i}\right)=L_{i}\left(p, \omega_{i}\right) \cos \theta_{i} d \omega_{i}
$$


where $L_{i}\left(p, \omega_{i}\right)$ is the radiance at the point $p$ from $\omega_{i}$ and $d \omega_{i}$ is the irradiance with direction $d \omega_{i}$ centered in $\omega_{i}$. The irradiance in the point $p$ from a solid angle $\Omega_{i}$ is obtained integrating $d E$ on $\Omega_{i}$ :

$$
E_{i}(p)=\int_{\Omega_{i}} L_{i}\left(p, \omega_{i}\right) \cos \theta_{i} d \omega_{i} .
$$

In the same way as ray-tracing is implemented in radio frequency models, reflections can be divided into mirror (specular reflection), diffuse and glossy (directive diffuse).

- Mirror Reflection: The specular reflection can be calculated with the BRDF as:

$$
f_{r}\left(\omega_{i}, \omega_{r}\right)=\frac{d L_{r}\left(\omega_{r}\right)}{L_{i}\left(\omega_{i}\right) \cos \left(\theta_{i} d \omega_{i}\right)},
$$

where $L$ is the radiance, $E$ is the irradiance and $\theta_{i}$ is the angle between $\omega_{i}$ and the normal $n$. The index $i$ denotes the incident light and $r$ the reflected light. The value of BRDF can be from 0 to infinity. The case of mirror type reflection is a special case of BRDF, where its value is infinite. The rendering equation for mirror reflection (Figure 3.9 (b)), can be expressed as:

$$
L_{r}\left(p, \omega_{0}\right)=L_{\text {direct }}\left(p, \omega_{0}\right)+L_{\text {indirect }}\left(p, \omega_{0}\right)
$$

where

$$
L_{\text {indirect }}\left(p, \omega_{0}\right)=\int_{2 \pi^{+}} f_{r}\left(p, \omega_{i}, \omega_{0}\right) L_{i}\left(p^{\prime},-\omega_{i}\right) \cos \theta_{i} d \omega .
$$

Equation (3.8) plays a fundamental role in ray-tracing because the value of the integral is related to the area where the ray tracer has to calculate the reflection.

\subsubsection{Shadows}

A shadow is defined as the parts of the scene that do not receive illumination from a light source because one or more objects block the illumination. In graphical computation, the basic calculation to define whether a point is in a shadow area has to first evaluate the visibility function $V\left(p, p^{\prime}\right)$, which is given by 


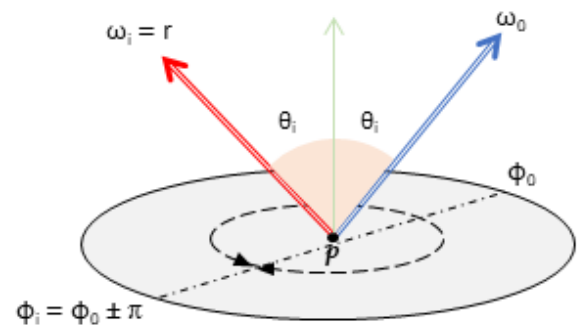

(a)

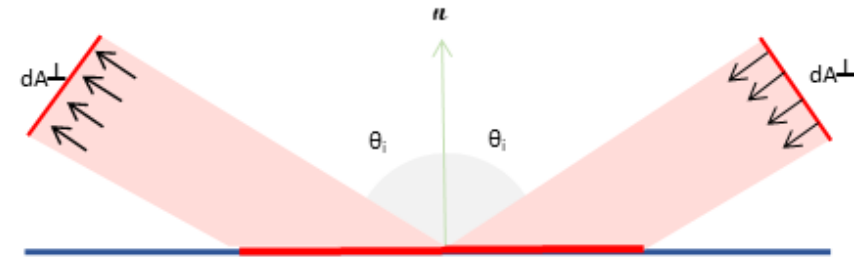

(b)

Figure 3.9: (a) Perfect light ray reflection (b) Perfect beam of reflected light

$$
\forall\left(p, p^{\prime}\right) \in A: V\left(p, p^{\prime}\right)= \begin{cases}1 & \text { if } \mathrm{p} \text { and p' can see each other } \\ 0 & \text { if } \mathrm{p} \text { and p' cannot see each other }\end{cases}
$$

where $p$ is the location of the light source and $p^{\prime}$ is the destination location. Therefore, the illumination reflected in a point with shadow is obtained from

$$
\left.L_{o}\left(p, \omega_{o}\right)=f_{r}(p, l(p)), \omega_{o}\right) l_{s} c_{l} V\left(p, l_{o}\right) \cos \left(\theta_{l}\right)
$$

where $l_{p}$ is the location of the light source and $p$ is the point of impact of the light beam.

\subsection{Object Properties}

\subsubsection{Materials and Shaders}

Materials in Unity are defined as a surface with rendering properties. Materials contain properties that are defined by the Shader that uses the material. A 
shader contains algorithms and mathematical methods to estimate the color of each pixel of the object according to the configuration of the material and the input value of the illumination [94]. In fact, shaders contain light-reflection calculations where the type of reflection is configurable. This configuration can correspond to specular reflections, diffuse, transparency, among others. Shaders behaviors can be modified by means of a script to achieve advanced lighting effects.

\subsubsection{Textures}

Textures are bitmap images that cover the 3D models in order to create a realistic effect on the visualization. The textures can contain information such as the reflectively of the material or even its roughness. These properties can be exploited together with the shaders to calculate the color of each pixel of the texture [94].

\subsection{User Interface}

Unity's User Interface (UI) is defined as the display area where the user can interact with the application. This area, also called Canvas, is defined within a 2D polygon. The Canvas is the area that all UI elements should be inside. The Canvas is a Game Object with a Canvas component on it, and all UI elements must be children of such a Canvas. The Canvas can contain information about the application, such as graphics, status variables and can also contain a set of tools that allow you to change values in the project variables.

The most common components for developing the graphical interface are:

- Button: A button has an onclick unit event that is settable.

- Toggle: This is an is-on checkbox that determines whether toggle is currently enabled or disabled. You can activate any event or function configured from a script.

- Slider: A slider allows you to set a range of values that are updated according to the position of the slider.

- Input field: An input field is used to make the text of a text element editable by the user.

- Dropdown: A dropdown has a list of options to choose from. A text string can be specified and set in the Inspector or dynamically from the code. 



\section{Chapter 4}

\section{Light Intensity Model for $5 \mathrm{G}$}

This chapter presents the Light Intensity Model (LIM) as a valid model for estimating large-scale propagation effects for the frequency bands above $6 \mathrm{GHz}$.

To that end, this chapter has been divided into the following sections:

- Section 4.1 introduces the motivation of LIM model for Millimeter Wave $(\mathrm{mmW})$ wireless propagation.

- Section 4.2 details the concept of LIM.

- Section 4.3 presents the 3D scenario of New York Univeristy (NYU) Manhattan campus developed in Unity.

- Section 4.4 explains the process of acquiring light intensity values for the LIM model.

- Section 4.5 presents the LIM calibration process and the light source variables values for 28 and $73 \mathrm{GHz}$ as a result of the calibration process.

- Section 4.6 describes the LIM calibration process and presents the light intensity parameters for 28 and $73 \mathrm{GHz}$ as a result of the calibration method.

- Section 4.7 presents the polynomial correlation functions of LIM that minimize the Root Mean Square Error (RMSE). 
- Section 4.8 compares the computational complexity between LIM and Real Time (RT) in terms of flops, Central Processing Unit (CPU) usage and time that each model takes.

- Section 4.9 draws the main conclusions of the chapter.

\subsection{Introduction}

Radio propagation in millimeter bands is prone to experiencing large variations in channel response due to blockage, which makes communication really challenging. Therefore, it is necessary to evaluate the possible candidate techniques that could make communications in the millimeter bands a reality. The use of realistic scenarios was motivated by the results obtained in the evaluation of stochastic and deterministic propagation loss models. The results have shown that it is beneficial to use realistic scenarios, especially in the higher frequency bands.

In the search for this precise characterization of a modern network, realistic propagation models based on ray-tracing are the preferred option, although their high computational cost prevents them from being adopted in system simulations. On the other hand, statistical propagation models, such as those proposed by Third Generation Partnership Project (3GPP) [75] and the NYU [40], which includes a synthetic 3D modeling. Such statistical models offer a good balance between complexity and precision, however, they fail to capture the real behavior of complex real environments. Therefore, it seems reasonable to propose the use of light engines, which essentially use ray-tracing algorithms, to estimate propagation losses in the radio channel.

\subsection{Light Intensity Model}

It is foreseeable that the implementation of Fifth Generation (5G) will operate in frequency bands that extend along the centimeter and millimeter wavebands. Radio signals at high frequencies suffer large signal variations given the high sensitivity to blockages and phenomena inherent to radio propagation that are a function of wavelength, requiring high precision 3D propagation models. Therefore, the combination of complex prediction models and accurate 3D scenarios makes computational time enormous. This is why less accurate stochastic propagation models have traditionally been used in radio communications. [70, 98, 99].

Within this framework, this work is based on the assumption that the use of General Purpose Graphics Processing Unit (GPGPU) can help to reduce the 
over-calculation time of propagation models based on ray-tracing given their high parallelization potential [70]. In computer graphics, ray-tracing algorithms were designed to illuminate or model 3D objects; however, these algorithms are being used in other fields. For example, the authors in [100] used a lighting tracker to calculate sound propagation over water or irregular terrain by modeling roads as non-linearities. In addition, the authors in [101] developed an outdoor radio wave propagation model based on ray-tracing. However, this model is limited to $2 \mathrm{D}$ calculations and it is not valid for realistic $3 \mathrm{D}$ scenarios.

Under this line, LIM is proposed as a propagation loss model, similar to a ray-tracing tool, based on the acquisition of light intensity at any point of interest in a video game engine that emulates a realistic 3D scenario, given the hypothesis that light and radio waves propagate similarly in the millimeter frequency bands.

\subsubsection{Concept Details}

Similar to propagation models based on ray-tracing, LIM relies on ray-tracing associated with light sources within 3D scenarios to calculate large-scale fade losses. LIM associates a normalized light intensity value, from a light source placed in the transmitter, with a propagation loss at any point of the scenario. Illumination conditions determine whether the measurement point is in an area with or without direct illumination, i.e. the points without direct view of the transmitter or Line of Sight (LoS). The light sources in Unity work in real time, which means that they illuminate the scene and the lighting is updated every frame. As a result, the lighting of the scenario is immediately updated when light sources or objects move within the scene. This property is of great interest to easily integrate the mobility of users in LIM. Also, pre-calculated light maps can be used to include both direct light that hits a surface as well as indirect light that is reflected from other objects or surfaces within the scene. These light or texture maps are calculated once and can be applied to objects to emulate the refraction of materials such as concrete, glass or gypsum panels. By adjusting the texture information, it is also possible to characterize the electromagnetic response of materials for a given frequency. This characterization of the materials has already demonstrated that it brings the results of the channel model closer to reality [98].

LIM takes advantage of the material modeling function to emulate the reflection losses of buildings for a given center frequency. Specifically, the scenario elements are covered with a layer of material emulating the materials, adapting the texture to wave reflectivity patterns in the visible spectrum to what could be expected in the low frequency domain. Figure 4.1 shows the irradiance, i.e. the amount of light reflected from the buildings on the stage. In this figure, 
the operating frequency is set at $28 \mathrm{GHz}$ and the coating layer emulates the concrete.

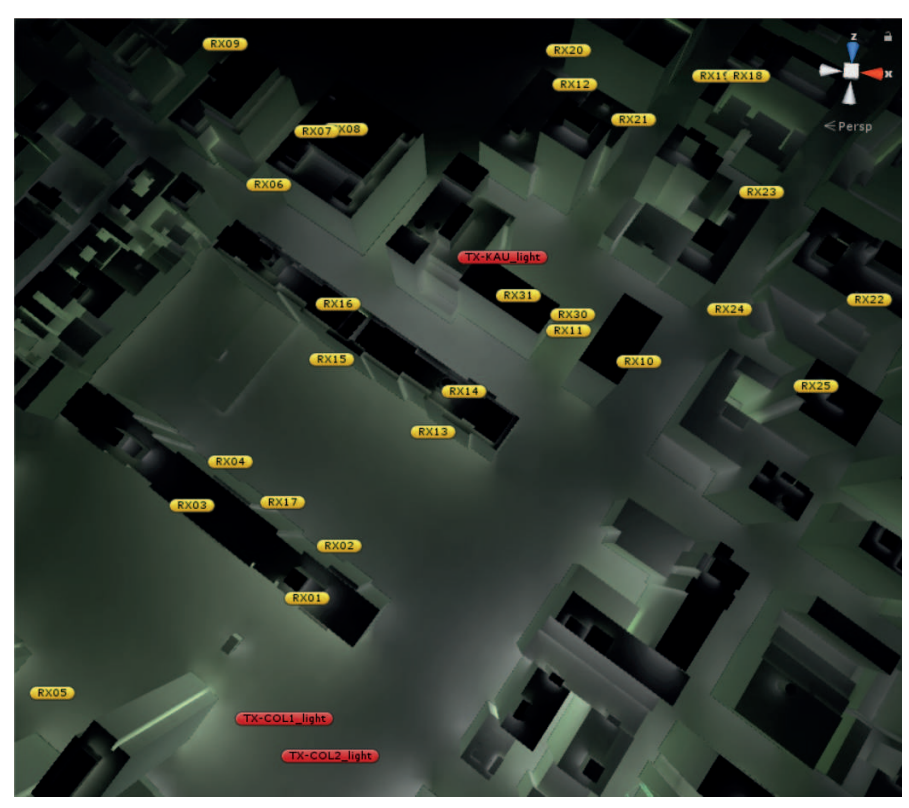

Figure 4.1: View of the amount of light reflected by buildings with a reflection layer configured for concrete

Diffraction phenomena can also be modeled with the Unity engine. The lighting techniques explained so far are not suitable for emulating diffraction. However, in an extension of LIM it is proposed to implement diffraction within the model, with the help of small points of light strategically placed at the diffraction points and behaving like isotropic antennas.

\subsection{Development of the Scenario of New York University in Unity}

Propagation simulation tools based on ray-tracing and stochastic models for wireless systems basically use synthetic scenarios, typically composed of a set of regular polygons distributed over a surface that seek to recreate the environment of the study scenario. With the Unity platform, this type of scenario can be easily recreated. However, our purpose is to go further and use one of Unity's 
advantages, which is the ease and ability to realistically reproduce a broad scenario with irregular shapes and accurately replicated details. For example, the use of geographic information databases such as Geographic Information System (GIS) can drive the model design process by introducing automation and at the same time make the resulting model geographically consistent [102].

In the example shown in the Figure 4.2, we transformed the information from GIS into a three-dimensional model of the New York University campus in downtown Manhattan. To obtain the 3D model, we imported the geographic information into Autodesk $3 \mathrm{ds}$ Max ${ }^{\circledR}$ and exported the model in Filmbox (FBX) format, which Unity understands. The resulting model accurately reproduced the shape of the buildings, although some post-processing exercises were required to improve the road network [103]. The combination of GIS and Unity data is quite novel, however, there are few contributions in the literature [104, 105].

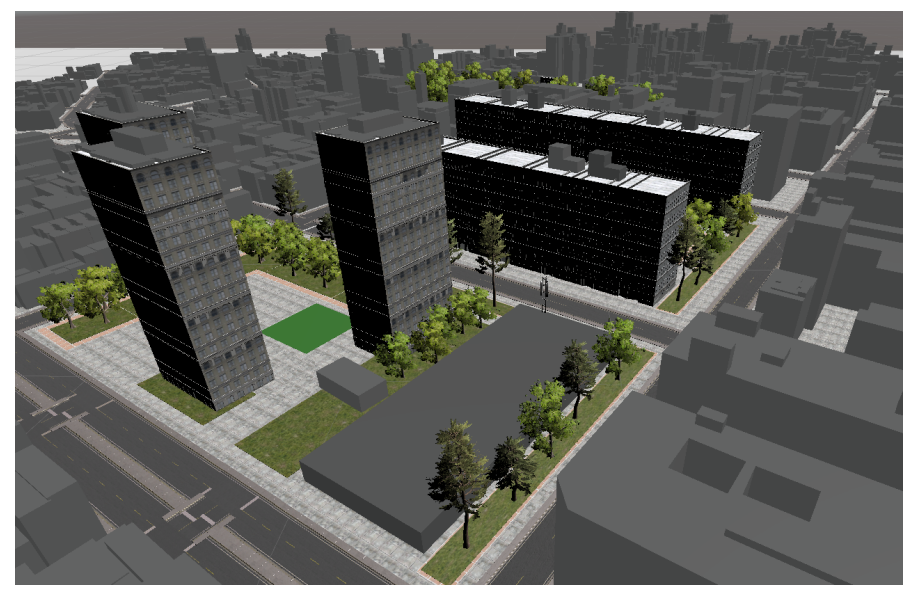

Figure 4.2: Creation of a realistic scenario in Unity

\subsection{Acquisition of Light Intensity Values}

As explained, LIM associates a normalized light intensity value, from a light source placed in the transmitter, with a propagation loss at any point in the scenario. The models used in the scenario must be very realistic in order not to have a negative influence on LIM. Objects with low definition could be misleading in the sense that shaded areas would not be accurate and the model would determine poorer propagation conditions than reality. Also, having sharper 


\section{CHAPTER 4. LIGHT INTENSITY MODEL FOR 5G}

edges would increase diffraction phenomena in the virtual scenario and could generate erroneous propagation estimates. For the acquisition of the intensity of light, cameras are located at the point of interest.

For the acquisition of the intensity of light, cameras are located at the points of interest. Cameras capture light, but they are directional in the sense that they only capture light in their field of view. To capture the light from all directions of the scene, the intensity of the light was obtained with the help of a sample plane, which is a plane $100 \times 100$ pixels in size, parallel to the ground. The sample plane was placed at the height of the user, which is usually $1.5 \mathrm{~m}$ above ground level in simulations of mobile networks. Indeed, a camera was placed and oriented towards the upper side of the sample plane, in such a way that the camera captures the luminance of the sample plane. The position of the camera is not a problem because the cameras are completely transparent and do not create shadow by themselves. The illumination on the lower side of the sample plane is very low, so its contribution to the large-scale loss value is negligible. The luminance or light intensity was then normalized to its maximum value, which is obtained when the light source is in the sample plane. With this strategy, we ensure that the camera captures light rays from all directions on the azimuthal plane.

\subsection{Measurement Campaign of Propagation Losses at 28 and $73 \mathrm{GHz}$ at NYU}

The propagation measurements used to calibrate the LIM model were performed by the NYU around the Manhattan campus from NYU [24, 49, 83, 84]. NYU provides the values of the onmidirectional propagation loss, as well as the GPS coordinates of the TX and RX locations available at [83]. Figures 4.3 and 4.4 show the distribution of the RXs for 28 and $73 \mathrm{GHz}$ respectively.

For measurements at $28 \mathrm{GHz}$, horn antennas with $24.5 \mathrm{dBi}$ gain and 10.9 degrees of aperture were used for both TX and Rx. Antenna heights range from $7 \mathrm{~m}$ to $17 \mathrm{~m}$ and the height of the receivers were placed at a standard height of $1.5 \mathrm{~m}$. The procedure for obtaining omnidirectional propagation losses consists of adding the total power received in the RX for each combination of TX-RX at each of the angles, and then eliminating the gains of both the TX and RX antennas [49]. Under the same procedure, measurements were made at $73 \mathrm{GHz}$, using horn antennas $27.0 \mathrm{dBi}$ gain and 7 degrees of aperture. 


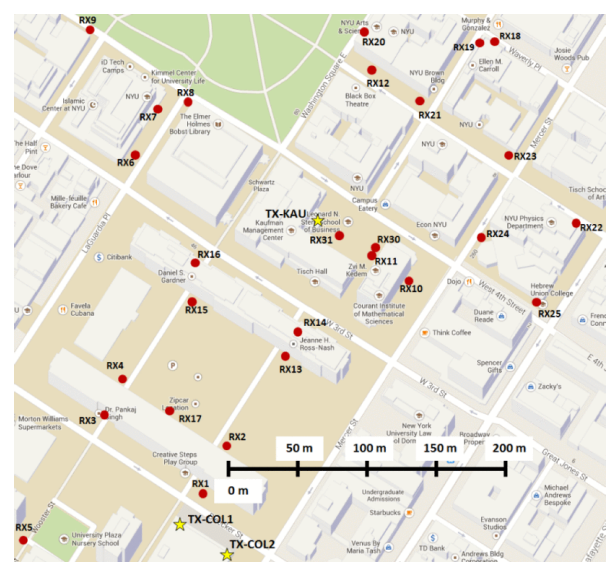

Figure 4.3: Locations of propagation loss measurements at $28 \mathrm{GHz}$ [40]

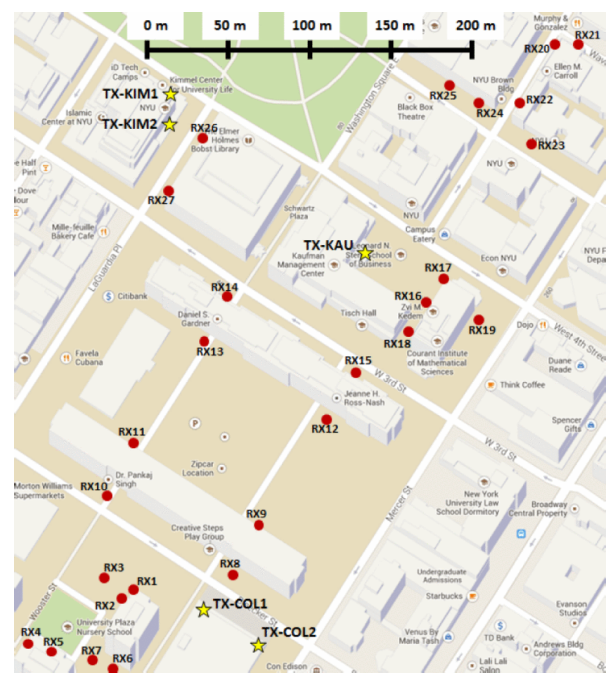

Figure 4.4: Locations of propagation loss measurements at $73 \mathrm{GHz}$ [40]

\subsection{Light Intensity Model Calibration}

For the calibration process of LIM we use the measurement campaigns of NYU [49]. Despite having a small collection of experimental measurement data, only 25 and 36 reference points at $28 \mathrm{GHz}$ and $73 \mathrm{GHz}$ respectively are the starting point for showing the benefits of LIM. From GIS data, a realistic 3D model was 
created, implementing both the geometric environment and the geographical references. Transmitters and receivers were placed in the synthetic scenario strictly following the location coordinates and the height and inclination specifications of the actual measurement campaign.

Omnidirectional light sources were placed one by one at transmitter locations and light intensity values were collected at receiving points. Some measurement points were randomly excluded from the calibration process to evaluate the approximation error at them. It is possible to observe the process of acquisition of the intensity of light in the capture of Figure 4.5.

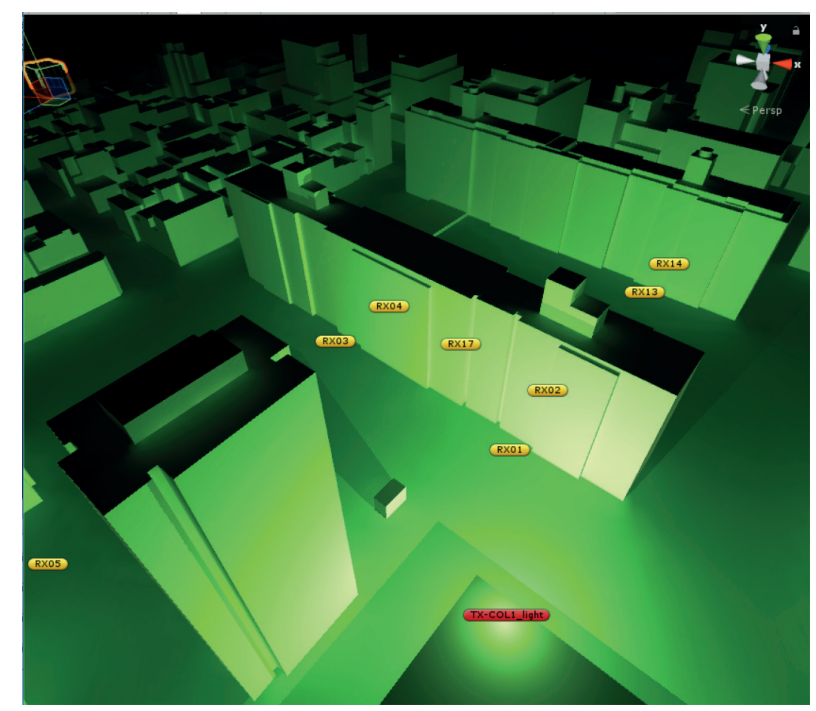

Figure 4.5: Light intensity acquisition - Calibration process

For the calibration process, the multivariate calibration method is used, which consists of evaluating all combinations of some of Unity's lighting variables to find the best correlation between light intensity values and real measurement data. The following four Unity lighting parameters were selected as calibration parameters: Light Source Intensity (LSI), Light Bounce (LB), Shadow Strength (SS) and Shadow Bias (SB) [94]. These four characteristics determine the light intensity values in the scenario. Briefly, LSI sets the brightness of diffuse ambient light in the scene. LB, determines the number of times reflection probes evaluate light ray bounces between objects. Finally, SS and SB determine how dark the shadows are and adjust the position and definition of the shadows, respectively. 
For each iteration, the calibration method LIM establishes the lighting conditions on the scenario and obtains the polynomial regression that best matches the light intensity pairs and large-scale reference fading values for LoS and Non Line of Sight (NLoS) separately. The procedure for calculating the best polynomial regression in a given lighting condition is illustrated in the flowchart in the Figure 4.6. To obtain the polynomial weights, the first step is to capture the normalized light intensity values at the reference points. Then, the light intensity and correlation of large-scale fading values are approximated with a polynomial regression, whose coefficients are chosen to minimize the RMSE of the estimation. Table 4.1 contains the optimized lighting parameters for the two frequencies.

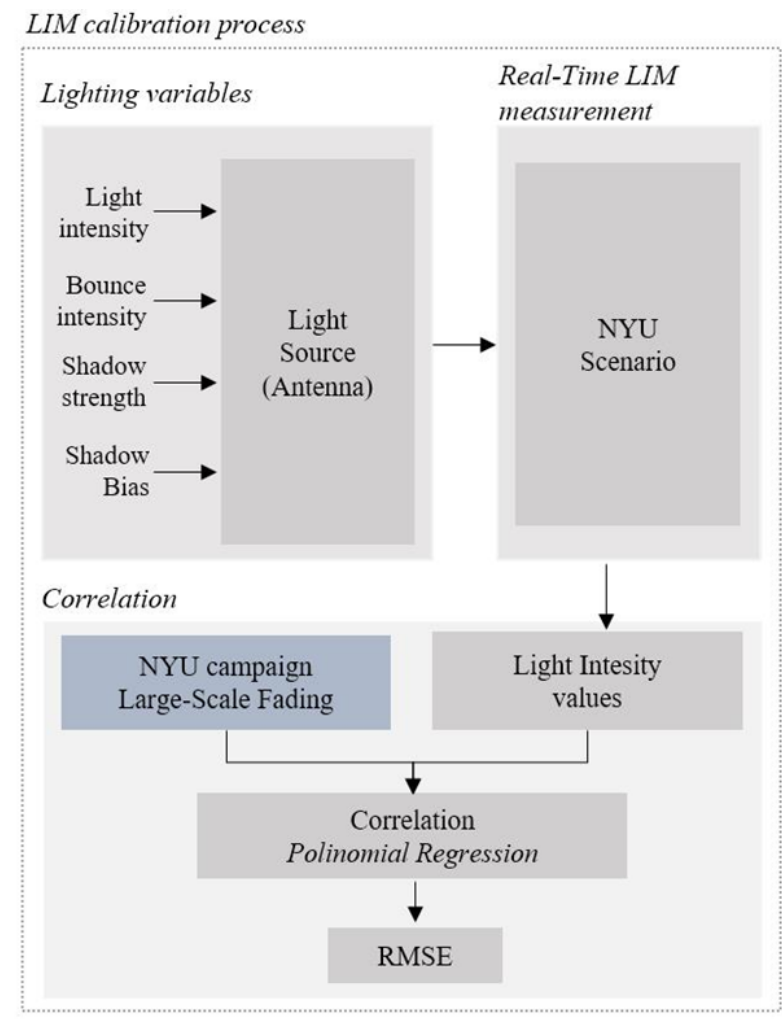

Figure 4.6: Calibration process flow chart

The pseudo-code of the calibration process is shown in Algorithm 1, where the outputs are: LSI, LB, SS, SB and the weights of the adjustment polynomial. 
Table 4.1: Calibration variable values for 28 and $73 \mathrm{GHz}$

\begin{tabular}{|c|c|c|}
\hline Lighting variables & $\mathbf{2 8} \mathbf{~ G H z}$ & $\mathbf{7 3} \mathbf{~ G H z}$ \\
\hline Light source intensity (LSI) & 2.75 & 2.15 \\
\hline Light bounce (LB) & 0.35 & 0.20 \\
\hline Shadow strength (SS) & 0.55 & 0.45 \\
\hline Shadow bias (SB) & 0.90 & 0.75 \\
\hline
\end{tabular}

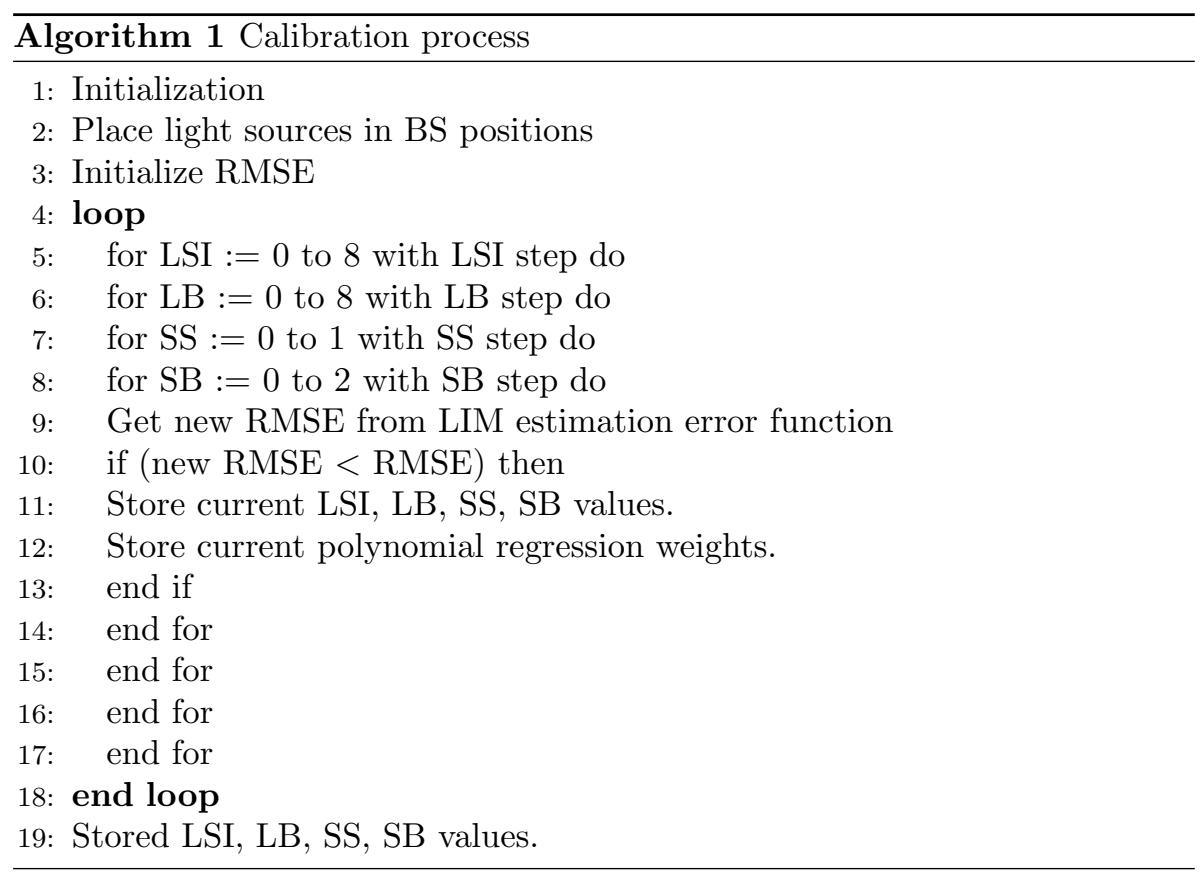

\subsection{Model Accuracy}

Large scale losses of NYU and light intensity points acquired from LIM were approximated with polynomial correlation functions for LoS and NLoS separately. For polynomial functions orders from 1 to 5 , and polynomial coefficients were chosen to minimize the estimate RMSE. Finally, the best adjustment was obtained with small polynomial orders. In (4.1) and (4.2) the best approximations to the propagation loss data at $28 \mathrm{GHz}$ for LoS and NLoS are provided, respectively: 


$$
\begin{gathered}
P L_{L o S}[d B]=-104.0 i+191.5, \\
P L_{N L o S}[d B]=75.7 i^{2}-123.7 i+164.3,
\end{gathered}
$$

and (4.3) and (4.4) provide the best approximations to the propagation loss data at $73 \mathrm{GHz}$ for LoS and NLoS respectively:

$$
\begin{aligned}
& P L_{L o S}[d B]=-91.94 i+185.0, \\
& P L_{N L o S}[d B]=-45.1 i+156.1,
\end{aligned}
$$

where $i$ is the normalized light intensity value.

Figure 4.7 shows the scatter plot of the large-scale loss, or path loss, and the normalized light intensity values for the 25 omnidirectional measurement data points at $28 \mathrm{GHz}, 6$ with $\mathrm{LoS}$ and 19 with NLoS. The figure also shows the model curves for the two frequencies under consideration.

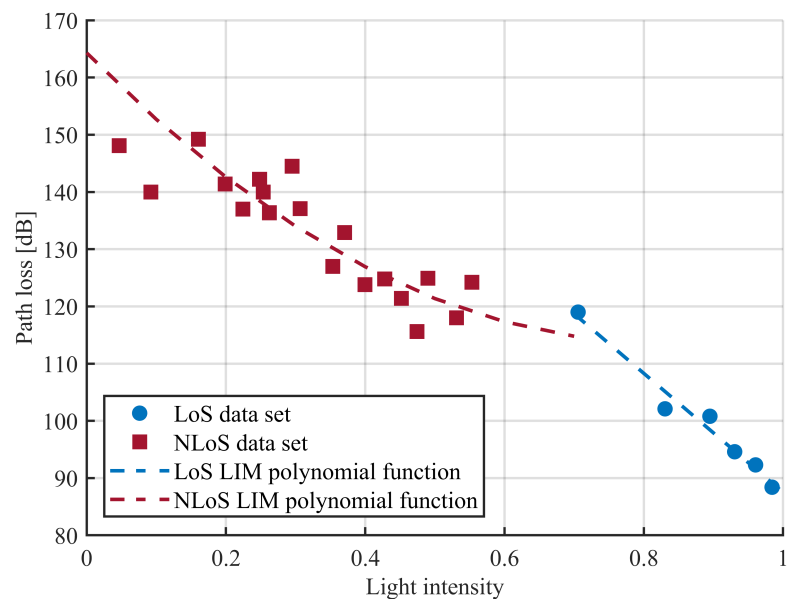

Figure 4.7: Scatter plot of propagation loss and light intensity correlation for $28 \mathrm{GHz}$ and LIM polynomial function for LoS and NLoS

In the same way, Figure 4.8 shows the scatter plot of the path loss and the normalized light intensity values for the 36 omnidirectional measurement data points at $73 \mathrm{GHz}, 8$ with $\operatorname{LoS}$ and 28 with NLoS. The figure also shows the model curves for the two frequencies under consideration.

In order to validate the calibration polynomial, the determination coefficient or R-square $R^{2}$ and the RMSE of the estimate were used. For $28 \mathrm{GHz}$ a value of $R^{2}$ of 0.97 and 0.75 for LoS and NLoS was used, respectively. And for 


\section{CHAPTER 4. LIGHT INTENSITY MODEL FOR 5G}

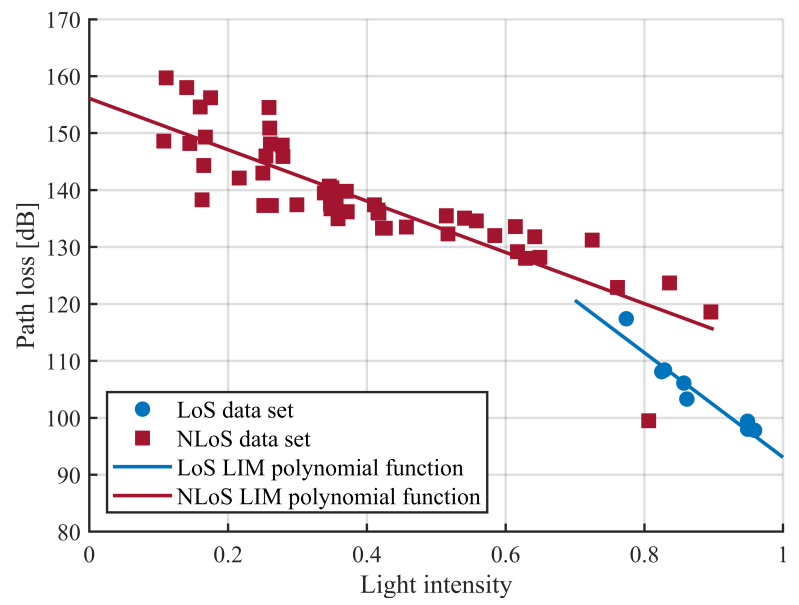

Figure 4.8: Scatter plot of propagation loss and light intensity correlation for $73 \mathrm{GHz}$ and LIM polynomial function for LoS and NLoS

$73 \mathrm{GHz}$, factors of 0.90 and 0.81 for LoS and NLoS were respectively used. To determine the RMSE, the measurement points that were excluded from the LIM calibration process (4 points at $28 \mathrm{GHz}$ and 6 at $73 \mathrm{GHz}$ ) were considered. LIM obtained RMSE values of 5.91 and $4.97 \mathrm{~dB}$ for 28 and $73 \mathrm{GHz}$ data, respectively. In view of these results, it can be said that the predictive accuracy of LIM is high. Also, it can be seen that there is a better adjustment in the $73 \mathrm{GHz}$ band, which suggests that for LIM the adjustment will be more precise the higher the operating frequency. This is appropriate and reasonable because wave propagation at high frequencies corresponds more to light propagation. In addition, considering that the $R^{2}$ value of 1 means a perfect match with the data, the $R^{2}$ values obtained with LIM indicates that the model is still perfectible.

Figures 4.7 and 4.8 also show that, for each frequency, there are two clearly separated point clouds in the scatter plot. LoS measurements, with large values of normalized light intensity and low loss on a large scale, are concentrated on the right side of the graph. On the other hand, all measurement points in NLoS, with low normalized light intensity values and high large-scale loss, are on the left side of the graph. Therefore, a realistic light engine like the one used by Unity takes advantage of the LoS / NLoS rating by taking into account the amount of light at the measurement point. Analyzing the estimation error in LoS and NLoS separately, we obtained that the RMSE with (4.1) in LoS is 1.31 $\mathrm{dB}$ and with (4.2) is $7.71 \mathrm{~dB}$ in NLoS, while the RMSE in $73 \mathrm{GHz}$ using (4.3) 
is $1.44 \mathrm{~dB}$ in LoS and with (4.4) is $6.25 \mathrm{~dB}$ in NLoS. The dispersion of data points in NLoS, along with LIM being less accurate in its estimation without direct vision, can be a side effect motivated by the simplifying assumption of having a single material layer covering the entire scenario. A more realistic characterization of each building material could make the LIM more accurate in its estimation.

Moreover, LIM has been compared with ray-tracing algorithms, to evaluate on the one hand, the precision with respect to the estimation of large-scale propagation losses and, on the other hand, the differences with respect to the computational complexity that is shown in detail in the Section 4.8. Table 4.2 shows that the difference between the two models is $1.49 \mathrm{~dB}$ and $0.71 \mathrm{~dB}$ for 28 and $73 \mathrm{GHz}$, respectively. As it can be seen, the smallest precision difference between LIM and ray-tracing is obtained with the highest frequency, which is the closest to the light frequency.

Table 4.2: RMSE for ray-tracing and LIM at 28 and $73 \mathrm{GHz}$

\begin{tabular}{|l|l|l|}
\hline \multicolumn{1}{|c|}{ Parameter } & Ray-tracing & \multicolumn{1}{c|}{ LIM } \\
\hline Global RMSE $[\mathrm{dB}] 28 \mathrm{GHz}$ & 5.65 & 7.14 \\
\hline Global RMSE $[\mathrm{dB}] 73 \mathrm{GHz}$ & 4.93 & 5.64 \\
\hline LoS RMSE $[\mathrm{dB}] 28 \mathrm{GHz}$ & 0.94 & 1.31 \\
\hline NLoS RMSE $[\mathrm{dB}] 28 \mathrm{GHz}$ & 6.19 & 7.71 \\
\hline LoS RMSE $[\mathrm{dB}] 73 \mathrm{GHz}$ & 0.93 & 1.44 \\
\hline NLoS RMSE $[\mathrm{dB}] 73 \mathrm{GHz}$ & 5.73 & 6.25 \\
\hline
\end{tabular}

\subsection{Computational Complexity}

Although LIM is based on ray-tracing algorithms that are processed in Graphics Processing Unit (GPU), the main benefit of using the LIM approach over typical ray-tracing algorithms needs to be emphasized, which is its low computational complexity and reasonably high accuracy. In that sense, this section compares LIM in terms of use of computing resources with a ray-tracing tool. In order to make this comparison, the ray-tracing tool was configured to consider maximum 3 reflections. Considering that the contribution of rays with more than 3 reflections is negligible in urban scenarios [106]. For a fairer com- 
parison with LIM, the ray-tracing tool did not calculate diffraction effects. In this experiment, the same virtual scenario is used and we repeat the measurement campaign as we did with LIM. The metric of evaluation was the average number of F-flops that LIM and ray-tracing needed to calculate the large-scale loss of a single measurement point. The flops were calculated as

$$
F=t_{\text {algorithm }} \cdot\left(G F L O P S_{\text {core }} \cdot N_{\text {cores }} \cdot C P U_{\text {usage }}\right),
$$

where $t_{\text {algorithm }}$ is the time that it takes for the algorithm to perform the calculation in seconds, and GFLOPS $S_{\text {core }}$ is the average use of the CPU while the algorithm is running. The calculation process was performed on a computer equipped with an Inter(R) Core (TM) i7-4720HQ processor that runs 8 cores in parallel $($ Ncores $=8$ ) at $2.60 \mathrm{GHz}$ each, with GFLOP Score $=3.51$. The resulting number of flops, and all other parameters in 4.5, for ray-tracing and LIM are shown in Table 4.3. As it can be seen, LIM is significantly faster than ray-tracing and shows two orders of magnitude less flops than ray-tracing. LIM takes advantage of Unity's efficient use of parallel cores for scene lighting to quickly approximate electromagnetic wave propagation.

Table 4.3: Calibration variable values for 28 and $73 \mathrm{GHz}$

\begin{tabular}{|l|l|l|}
\hline \multicolumn{1}{|c|}{ Parameter } & \multicolumn{1}{|c|}{ Ray-tracing } & \multicolumn{1}{c|}{ LIM } \\
\hline$t_{\text {algorithm }}$ & 732.80 & 9.30 \\
\hline$C P U_{\text {usage }}$ & 0.12 & 0.07 \\
\hline Flops $(\mathrm{F})$ & $2.48 \cdot 10^{9}$ & $1.91 \cdot 10^{7}$ \\
\hline RMSE $[\mathrm{dB}] 28 \mathrm{GHz}$ & 5.65 & 7.14 \\
\hline RMSE $[\mathrm{dB}] 73 \mathrm{GHz}$ & 4.93 & 5.64 \\
\hline
\end{tabular}

\subsection{Conclusions}

In this chapter, LIM was presented as a model for the estimation of propagation losses based on scene lighting conditions for the $\mathrm{mmW}$ bands. Its results are very similar to the ray-tracing ones but with a substantially lower computational complexity. The predicted model can also accurately reproduce the geometry of an urban area, making this model a solid choice for evaluating future $5 \mathrm{G}$ technologies in realistic scenarios. However, the model has scope 
for improvement. The results presented have been obtained with a uniform layer of material. The inclusion of a wider range of materials and a more precise parameterization that could capture the reflection and diffuse scattering properties of the material with greater precision at different frequencies would probably decrease the estimation error of the model. LIM is a strong candidate to be considered as a path loss model for complex scenarios and real-time simulations where ray-tracing algorithms are highly computationally expensive, thanks to multiple GPGPUs running in parallel and assuming that the complexity required is similar to lighting a scene in any conventional $3 \mathrm{D}$ video game. 



\section{Chapter 5}

\section{Extension of the Light Intensity Model}

This chapter presents an extension of the Light Intensity Model (LIM) in which the materials used within the game engines are characterized in a different way, by configuring the normal map of their surfaces and their scattering and reflection functions, to improve the matching between the predictions of LIM.

To that end, this chapter has been divided into the following sections:

- Section 5.1 introduces the importance of diffuse scattering for Millimeter Wave $(\mathrm{mmW})$ wireless propagation.

- Section 5.2 details the diffuse scattering measurement campaign carried out in this Thesis.

- Section 5.3 presents the calibration of the ray-tracing models with diffuse scattering based on the measurement campaign.

- Section 5.4 presents the calibration of the LIM model with diffuse scattering based on the measurement campaign.

- Section 5.5 draws the main conclusions of the chapter.

\subsection{Introduction}

Diffuse scattering can be described as a special case of reflection that occurs when a radio wave strikes irregular objects that are smaller in comparison to their wavelength. While the intensity of the scattered rays is generally low 


\section{CHAPTER 5. EXTENSION OF THE LIGHT INTENSITY MODEL}

when compared to the reflected signal, they can provide a gain in fading on a small scale [22].

Diffuse scattering has been widely studied for frequencies below $6 \mathrm{GHz}$. In [107], Degli-Esposti et al. conducted a measurement campaign at $1296 \mathrm{MHz}$ in which the scattering pattern of a concrete surface was obtained. The measurements were compared with Lambertian and Directive models, reaching Root Mean Square Error (RMSE) of up to 1.85 dB. Degli-Esposti et al. concluded in this work that there is a need to consider diffuse scattering in urban environments and that the contribution of scattered components to the total power can be even higher than that of the specular reflections in some points. It also suggests that the $\mathrm{S}$ parameter of the Lambertian and Directive model will increase in such urban environments. In the same sense, in [108], DegliEsposti et al. conducted measurements in the $1800 \mathrm{MHz}$ band in Urban Macrocell (UMa) and Urban Micro-cell (UMi) environments. In UMa, the impact of diffuse scattering is very important. The location of the TX, which is higher than the surrounding buildings, considerably increases the points where diffuse scattering occurs. In fact, it is confirmed that diffuse scattering in distant locations reaches power levels greater than the reflections. In UMi environments, a greater amount of diffuse scattering is generated due to the characteristic large number of elements of a UMi environment. Therefore, the components of diffuse scattering must be considered especially in this type of scenario. In the $2.4 \mathrm{GHz}$ band, [109] presents a diffuse scattering model that extends the Effective-Roughness (ER) model with forward diffuse scattering. This model was compared with measurements, where it is shown that diffuse scattering is relevant even on smooth surfaces.

For the band above $6 \mathrm{GHz}$, several studies have been carried out. In [110], measurements have been made from 1 to $10 \mathrm{GHz}$ for different building materials with different roughness levels. The results show that diffuse scattering begins to have a greater impact as frequency increases, especially for rougher materials. The authors of [111] made a parameterization of the diffuse scattering models for the $60 \mathrm{GHz}$ band for 3 materials considering their electrical characteristics as well as their roughness. As a result, the values of the $\mathrm{S}$ parameter were obtained for the Lambertian and Directive models. In [112], measurements were made in the $26 \mathrm{GHz}$ band to compare different ray-tracing model. In their results, the authors show the importance of diffuse scattering implementation. In fact, it can be observed that at some Non Line of Sight (NLoS) points, the differences between the measured path loss values and the ray-tracing model that includes diffuse scattering are better suited compared to the ray-tracing model without diffuse scattering. RMSE can go from more than $20 \mathrm{~dB}$ to less than $5 \mathrm{~dB}$ when diffuse scattering is implemented (see Figure 5 of [112]). In 
addition, the results estimate new $\mathrm{S}$ coefficients for Lambertian and directive models.

In this context, and given the importance the diffuse scattering components in the bands above $6 \mathrm{GHz}$, this Thesis has considered and extension of the LIM model in which the materials used in game engines are characterized properly to produce an appropriate diffuse scattering of the light. To make such an extension of LIM, first a measurement campaign has been carried out in a controlled scenario to characterize the scattering pattern of several materials. This characterization is then used to calibrate both ray-tracing and LIM, whose accuracy to predict channel losses is then compared.

\subsection{Diffuse Scattering Measurement Campaign at 26 to $40 \mathrm{GHz}$}

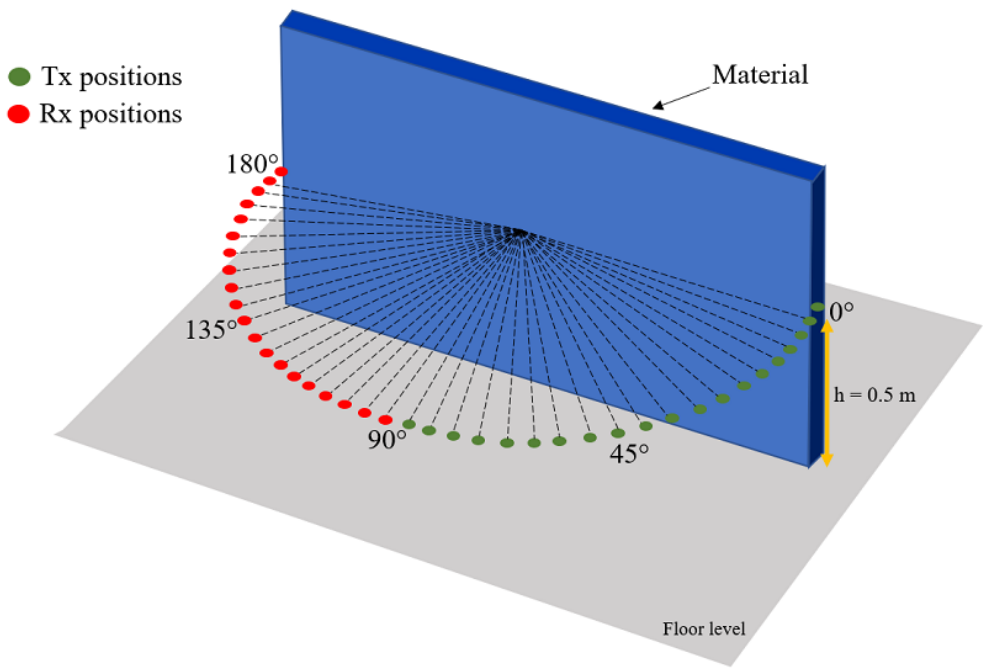

Figure 5.1: Measurement setup scheme for TX and RX positions

For the measurement campaign, the scenario shown in the Figure 5.1 has been implemented. TX and RX were equipped with a horn antenna manufactured by Flann Microwave, model 22240-20, operating on frequencies from 26 to $40 \mathrm{GHz}$, with a nominal gain of $20 \mathrm{dBi}$. The beamwidth at $3 \mathrm{~dB}$ is $18 \mathrm{de}-$ grees for both the vertical and horizontal planes. They are connected to ports 1 and 2 of the VNA model E8363A. 18 positions have been considered for the 
TX and 18 positions for the RX, from 0 to 90 degrees for the TX and from 90 to 180 degrees for the RX. Adjacent positions are separated by 5 degrees. We did not use an anechoic chamber and that could be, then, an error source. To minimize that error, potential sources of reflected rays were far from the material under test. The nearest surface was the floor, which was at $50 \mathrm{~cm}$ from the antennas. In addition, the directivity of the antennas and the use of $\mathrm{mmW}$ minimizes the probability of having reflected rays with significant power coming from materials different to the material under test.

For the measuring process, the TX is set at the first measuring point ( 0 degrees) pointing to the center of the material, at a height of $0.50 \mathrm{~m}$. Then, a sweep is made with the RX in the positions of the semicircle, from 90 to 180 degrees. This procedure is repeated until all the combinations between TX-RX are obtained. For the positioning and pointing of the antennas, lasers have been used, as shown in the Figure 5.2.

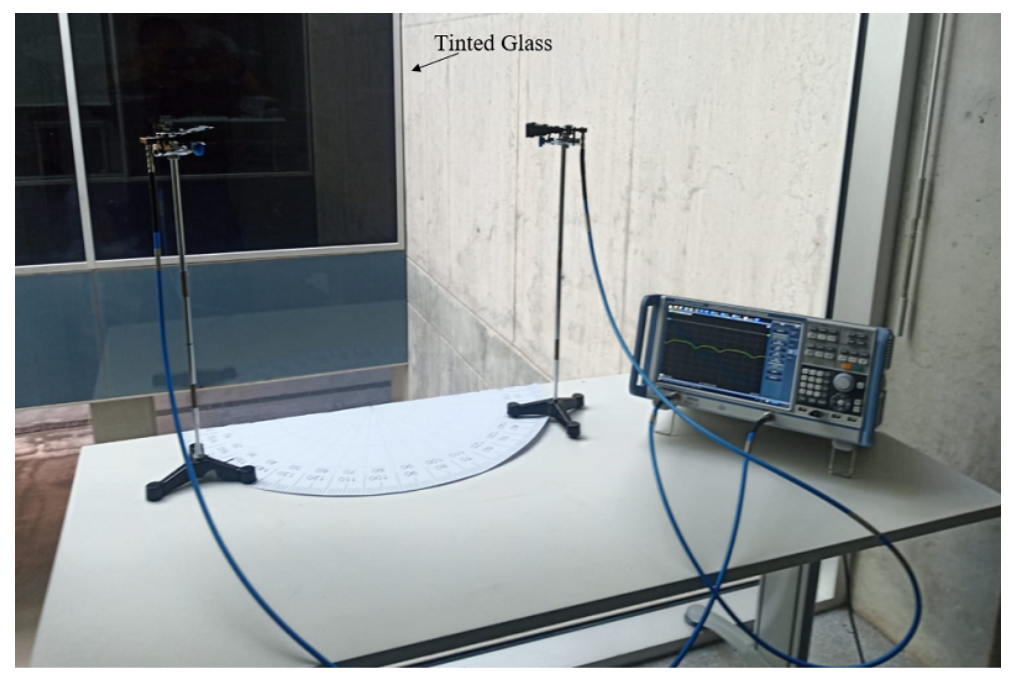

Figure 5.2: Diffuse scattering measurement setup

Materials measured were concrete, chipboard, tinted glass and metal. Figures 5.3, 5.4 and 5.5 show the results obtained in the whole frequency range between $26 \mathrm{GHz}$ to $40 \mathrm{GHz}$ for a fixed TX position at 60 degrees with all its RX combinations. For all materials it can be seen that the highest power level corresponds to the angle of the specular reflection. In fact, the other components are mainly the product of diffuse scattering. 


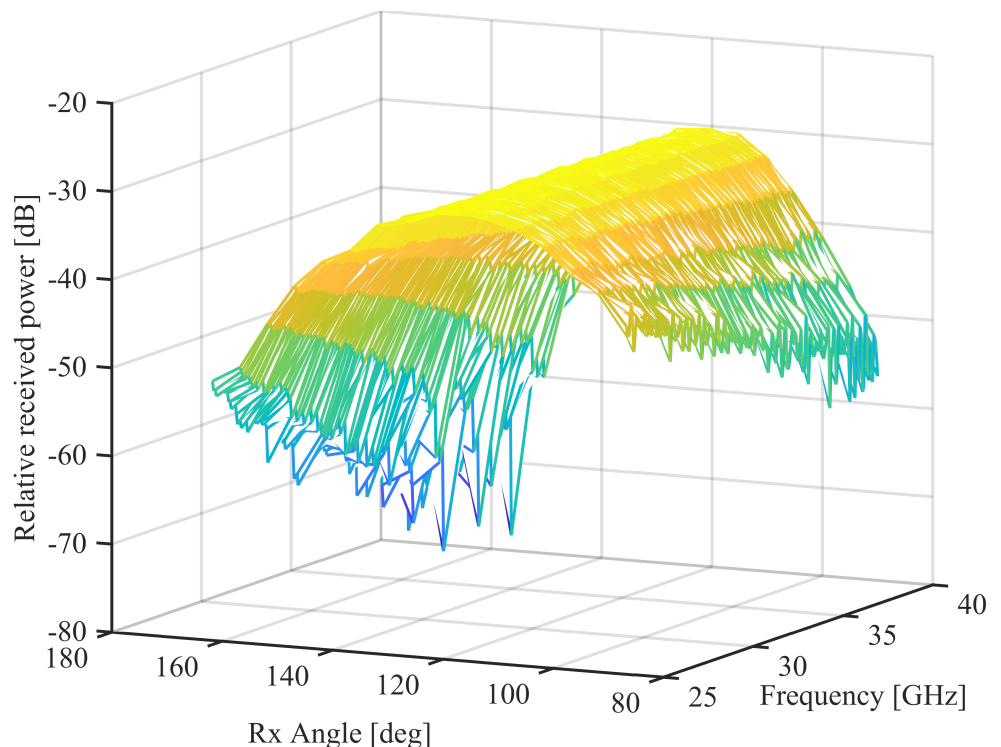

Figure 5.3: Measurement results for metal. TX position at 60 degrees

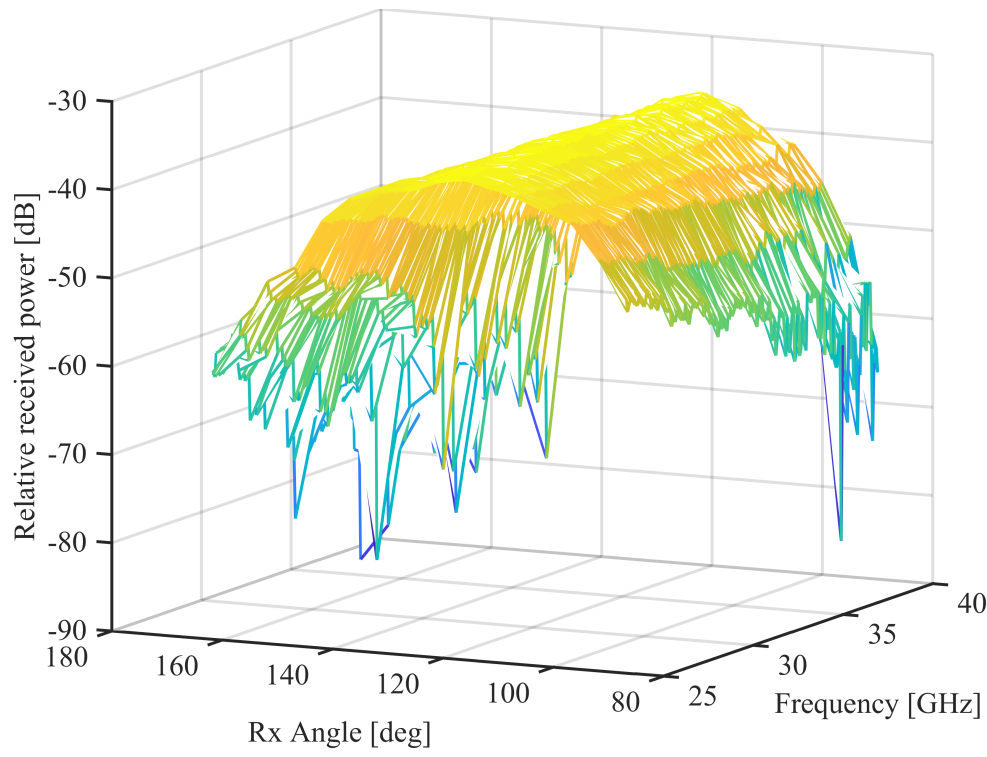

Figure 5.4: Measurement results for concrete. TX position at 60 degrees 


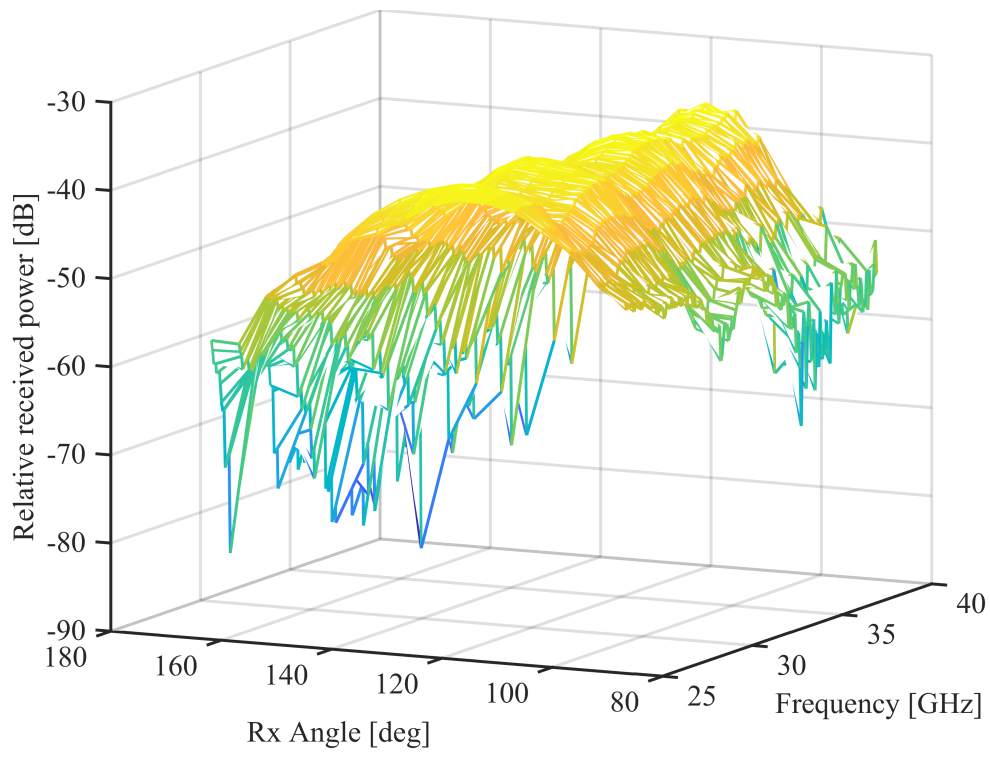

Figure 5.5: Measurement results for chipboard. TX position at 60 degrees

\subsection{Ray-Tracing Calibration}

The objective of this analysis is to validate the measurements obtained using ray-tracing algorithms. To calculate the losses among TX and RX, we used

$$
P L=10 \log _{10}\left(P_{T X}\right)-10 \log _{10}\left(P_{R X}\right),
$$

where $P_{T X}$ is the TX power in watts $(\mathrm{W})$, and $P_{R X}$ is the received power for a given field strength. $P_{R X}$, can be expressed as

$$
P_{R X}=10 \log _{10}\left(\sum_{i=0}^{N} E_{\text {ray }_{i}}\right)-20 \log _{10}\left(\frac{f}{10^{9}}\right)-167.2,
$$

where $E_{\text {ray }_{i}}$ is the sum of electric field due all the multi-path components, $f$ the frequency in $\mathrm{GHz}$ and $N$ is the total amount of rays. It is considered that infinitesimal rays are emitted from the TX antenna in all directions with a gain in power relative to the radiation pattern of the antenna. Therefore, the power of each ray is:

$$
P_{\text {ray }}=\frac{P_{T X}}{4 \pi(\delta \Omega)^{2}}
$$




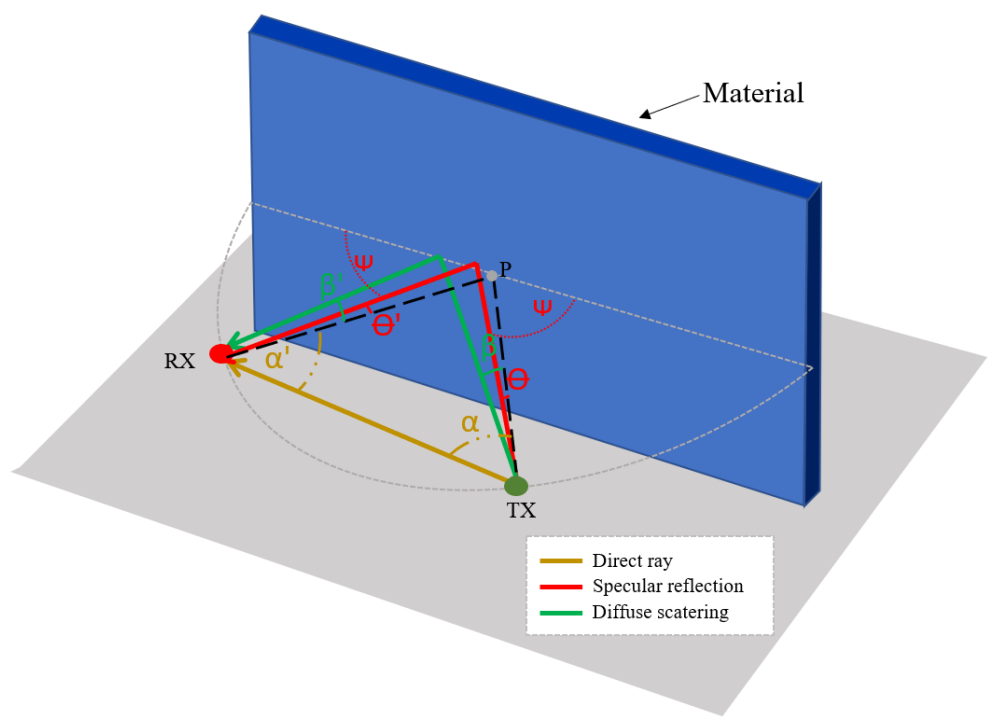

Figure 5.6: Geometry of the measurement setup

where $\delta \Omega$ is the angle covered by each ray.

Figure 5.6, shows a single bounce situation, where TX and RX antennas are oriented to point $P$. Due to the antenna aperture, the components present correspond in greater magnitude to the rays of specular and diffuse reflection. And, components of the direct rays in lower magnitude. The total electric field $E_{t}$ at RX point could be defined as:

$$
E_{t}=E_{d}+E_{r}+E_{s}
$$

where $E_{d}, E_{r}$ and $E_{s}$ are the components due direct loss, specular reflection and diffuse scattering. To calculate the electric field due the direct component:

$$
\begin{gathered}
E_{d}=E_{0} \sqrt{G_{R X}(\alpha)} e^{\frac{-j 2 \pi d}{\lambda}}, \\
E_{0}=\frac{\sqrt{30 P_{t_{\text {ray }}} G_{T X}(\alpha)}}{r},
\end{gathered}
$$

where $G_{R X}\left(\alpha^{\prime}\right)$ is the RX antenna gain in $\alpha$ direction. $E_{0}$ is the field strength due to the TX. $2 \pi / \lambda$ is the wave constant in free space, $G_{T X}(\alpha)$ is the TX antenna gain, $r$ is the distance between TX and RX, and $P_{t_{\text {ray }}}$ is the ray power in watts $(\mathrm{W})$. 
Specular component is calculated using Snell's laws of reflection. The reflection coefficient $\Gamma$ is a relation between the incident and reflected waves that depends on the electric characteristics of the material. So, the specular electric field is calculated as:

$$
E_{r}=E_{0} \Gamma \sqrt{G_{R X}(\theta)} e^{\frac{-j 2 \pi d}{\lambda}} .
$$

Note that $G_{R X}\left(\theta^{\prime}\right)$ is the RX antenna gain in $\theta^{\prime}$ direction (specular reflection). $\Gamma_{h}$ and $\Gamma_{v}$ are the reflection coefficients for horizontal and vertical polarization, respectively.

$$
\begin{gathered}
\Gamma_{h}=\frac{\sin \psi-\sqrt{\varepsilon^{*}-\cos ^{2} \psi}}{\sin \psi+\sqrt{\varepsilon^{*}-\cos ^{2} \psi}}, \\
\Gamma_{v}=\frac{\varepsilon^{*} \sin \psi-\sqrt{\varepsilon^{*}-\cos ^{2} \psi}}{\varepsilon^{*} \sin \psi+\sqrt{\varepsilon^{*}-\cos ^{2} \psi}},
\end{gathered}
$$

where $\varepsilon^{*}$ is the relative complex dielectric constant and is defined by:

$$
\varepsilon^{*}=a \cdot f^{b}-j 17.98 \frac{c \cdot f^{d}}{f} .
$$

Variables a, b, c and d are the electric characteristics of the materials, shown in Table 5.1 [73]. The real part represents the relative permittivity and the imaginary part the complex conductivity.

\begin{tabular}{|c|c|c|c|c|c|}
\hline \multirow{2}{*}{ Material } & \multicolumn{2}{|c|}{ Permittivity } & \multicolumn{2}{c|}{ Conductivity } & Frequency \\
\cline { 2 - 6 } & $\mathbf{a}$ & $\mathbf{b}$ & $\mathbf{c}$ & $\mathbf{d}$ & $\mathbf{G H z}$ \\
\hline Concrete & 5.31 & 0 & 0.0326 & 0.8095 & $1-100$ \\
\hline Metal & 1 & 0 & $10^{7}$ & 0 & $1-100$ \\
\hline Glass & 6.27 & 0 & 0.0043 & 1.0718 & $0.1-100$ \\
\hline Chipboard & 2.58 & 0 & 0.0217 & 0.7800 & $1-100$ \\
\hline
\end{tabular}

Table 5.1: Material properties up to $100 \mathrm{GHz}$ [73]

Finally, the electric field contribution due diffuse scattering, $E_{s}$, is evaluated adopting the diffuse scattering models presented in $[113,114]$. Due to the fact that we are going to evaluate several models, The equation (5.11) has been proposed for the calculation of $E_{s}$, defined as the scattering electric field obtained from the Lambertian or Directive model, and $G_{R X}(\beta)$ being the RX antenna gain.

$$
E_{s}=\sqrt{G_{R X}(\beta)} E_{s^{\prime}} .
$$

The geometry to explain diffuse scattering models is shown in Figure 5.7. 


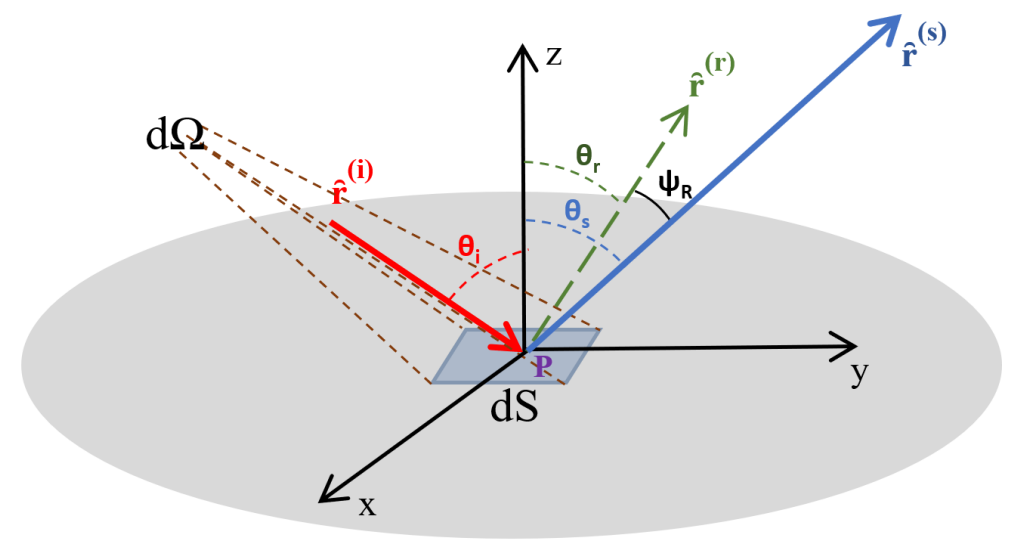

Figure 5.7: Generic geometric model of reflection and diffuse scattering

In this model, in $P$, the point where diffuse scattering occurs, it has associated a coefficient of dispersion $S$ and a reflection coefficient $\Gamma$. In [113] two approaches of $S$ are defined. In the first one all incident power is scattered and spreads in all directions. In the second approach, the scattering components also spreads in all directions but only considering the reflected energy. The first one, $S$ is defined by:

$$
S=\left.\frac{\left|\bar{E}_{S}\right|}{\left|\bar{E}_{i}\right|}\right|_{d S},
$$

where $E_{s}$ is the amplitude of the electric field of diffuse scattering, $E_{i}$ is the amplitude of the specular electric field and $P$ is the point where diffuse scattering is produced. The power balance due diffuse scattering, reflection and transmission in $P$ is defined as follows [115]:

$$
1=\Gamma^{2} R^{2}+S^{2}+\frac{P_{p}}{P_{i}},
$$

where $\mathrm{R}$ defined in equation (5.14) is the reflection loss factor, $0<R<1, \Gamma$ and $S$ are the scattering and reflection coefficient respectively, $P_{p}$ is the power that penetrate the material and $P_{i}$ is the incident power.

$$
R \cong \sqrt{1-\frac{S^{2}}{\Gamma^{2}}}
$$


In the second approach, $S$ is defined by equation (5.15) and the power balance is defined by (5.16).

$$
\begin{gathered}
S=\left.\frac{\left|\bar{E}_{S}\right|}{\left|\bar{E}_{r}\right|}\right|_{d S}, \\
1=\Gamma^{2}\left(R^{2}+S^{2}\right)+\frac{P_{p}}{P_{i}} .
\end{gathered}
$$

With these two approaches, a function $U$ is defined as follows:

$$
U= \begin{cases}1 & \text { if } S=\left|\bar{E}_{S} / \bar{E}_{i}\right| \text { (Def. 1) } \\ \Gamma & \text { if } S=\left|\bar{E}_{S} / \bar{E}_{R}\right| \text { (Def. 5). }\end{cases}
$$

In order to evaluate the measurements of the campaign, two diffuse scattering models are considered. The Lambertian model and the Directive model. The geometry shown in Figure 5.7 is valid for both models.

\section{A. Lambertian Model}

In the Lambertian model, energy is scattered in all directions centered in the norm of the point $P$. In this model, the module of the diffuse electric field is defined by:

$$
\left|\bar{E}_{S}\right|^{2}=\left(\frac{K_{0} \cdot S}{r_{i} \cdot r_{s}}\right)^{2} \cdot U^{2} \cdot \frac{\cos \left(\theta_{i}\right) \cdot \cos \left(\theta_{s}\right)}{\pi} d S,
$$

where $r_{i}$ is the distance between the $T X$ and the point $P, r_{s}$ is the distance between RX and the point $P . \theta_{i}$ and $\theta_{s}$ are the incident and departure angles, and

$$
K_{0}=\sqrt{\left.60 G_{T X}(\beta) P_{t_{\text {ray }}}\right)},
$$

where $G_{R X}(\beta)$ is the antenna gain in $\beta$ direction, and $P_{t_{\text {ray }}}$ is the ray power in watts.

B. Directive Model

In the directive model, energy is scattered in the directions around reflection angles. It has two variants, directive and directive with back-scatter. The module of the electric field in the directive model is:

$$
\left|\bar{E}_{S}\right|^{2}=E_{S 0}^{2} \cdot\left(\frac{1+\cos \psi_{R}}{2}\right)^{\alpha_{R}},
$$


where $\psi_{R}$ is the angle between the scattering and specular rays, the exponent $\alpha_{R}$ is related to the width of the scattering lobe; the lobe will be wider when the exponent is greater. $E_{S 0}^{2}$ is defined by

$$
E_{S 0}^{2}=\left(\frac{S K_{0}}{r_{i} r_{s}}\right)^{2} U^{2} \frac{d S \cos \left(\theta_{i}\right)}{F_{\alpha_{R}}},
$$

where $F_{\alpha_{R}}$ is

$$
F_{\alpha_{R}}=\frac{1}{2^{\alpha_{R}}} \cdot \sum_{j=0}^{\alpha_{R}}\left(\begin{array}{c}
\alpha_{R} \\
j
\end{array}\right) \cdot I_{j}
$$

and $I_{j}$ is

$$
I_{j}=\frac{2 \pi}{j+1} \cdot\left[\cos \theta_{i} \sum_{w=0}^{\frac{j-1}{2}}\left(\begin{array}{c}
2 w \\
w
\end{array}\right) \cdot \frac{\sin ^{2 w} \theta_{i}}{2^{2 w}}\right]^{\frac{1-(-1)^{j}}{2}}
$$

\subsubsection{Analytical Results}

In this section the comparisons between the measurements and the estimations obtained with the ray-tracing algorithms are shown. To analyze the results we will focus on the $28 \mathrm{GHz}$ band. The figures show the relative received power over the entire range of RX positions for a fixed TX position. Both TX and RX antennas have been previously characterized for the horizontal and vertical planes. The geometry shown in Figure 5.1 is considered. Following the method used in [115], the measurements have been adjusted using the two definitions (Def. 1 and Def. 5 model) shown in (5.17), in order to adjust the parameter $\mathrm{S}$ of the diffuse scattering components. The following figures show the results for different combinations of TX-RX at $28 \mathrm{GHz}$.

It can be noted from the figures that for metal, specular components are the most dominant. In fact, since this material is very reflective, the value of the parameter $S$ that best fits the measurements is between 0 and 0.1 with the directive Def. 5 model. For concrete, when the TX is in positions between 20 and 40 degrees, $S=0.1$ better fits the measured values, with the directive Def 1 model, possibly due to the roughness of the material. In the same way, it can be observed that the curves that fit most for the chipboard are the directive Def1 model. with $S=0.1$. Finally, for tinted glass, the Def. 5 directive model adjusts more to the values measured with $S=0.2$. Table 5.2 shows the RMSE between the measured values, for each of the materials.

Based on the results obtained, it can be indicated that values of relative received power from the measurement campaign are valid to be used in the extension of the LIM model proposed in this chapter. 
CHAPTER 5. EXTENSION OF THE LIGHT INTENSITY

MODEL

Table 5.2: Measurement analysis results for $28 \mathrm{GHz}$

\begin{tabular}{|l|l|l|l|}
\hline Material & $\begin{array}{l}\text { Diffuse Scatter- } \\
\text { ing Model }\end{array}$ & S & $\begin{array}{l}\text { RMSE [dB] } \\
\text { Ray-Tracing }\end{array}$ \\
\hline Metal & Directive Def. 5 & $0-0.1$ & 2.78 \\
\hline Concrete & Directive Def. 1 & 0.1 & 2.96 \\
\hline Chipboard & Directive Def. 1 & 0.1 & 2.67 \\
\hline Tinted glass & Directive Def. 5 & 0.2 & 2.77 \\
\hline
\end{tabular}

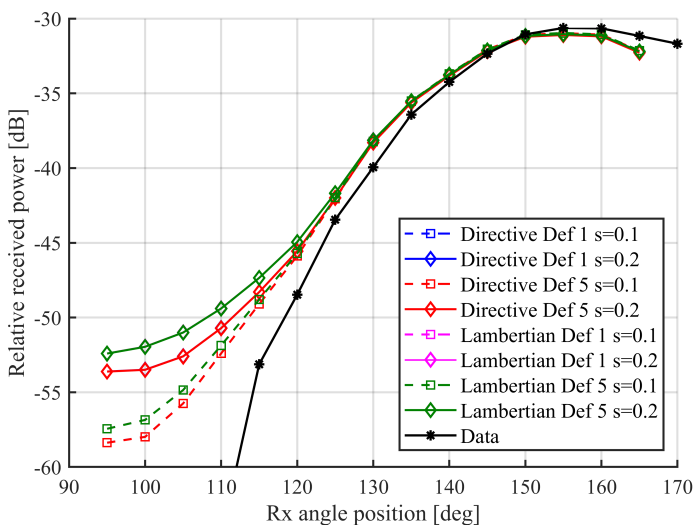

Figure 5.8: Ray-tracing results for metal @28 GHz, TX at 30 degrees

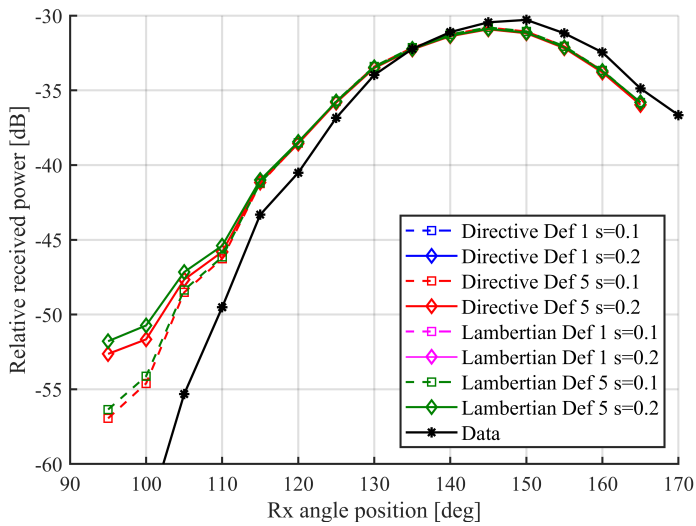

Figure 5.9: Ray-tracing results for metal @28 GHz, TX at 40 degrees 


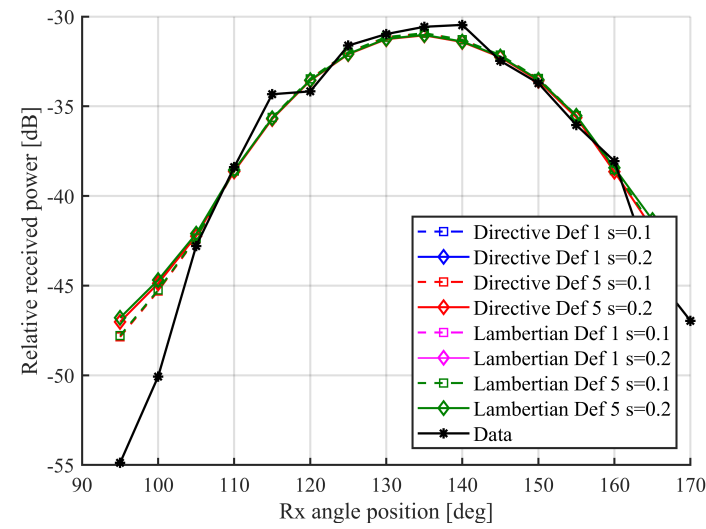

Figure 5.10: Ray-tracing results for metal @28 GHz, TX at 50 degrees

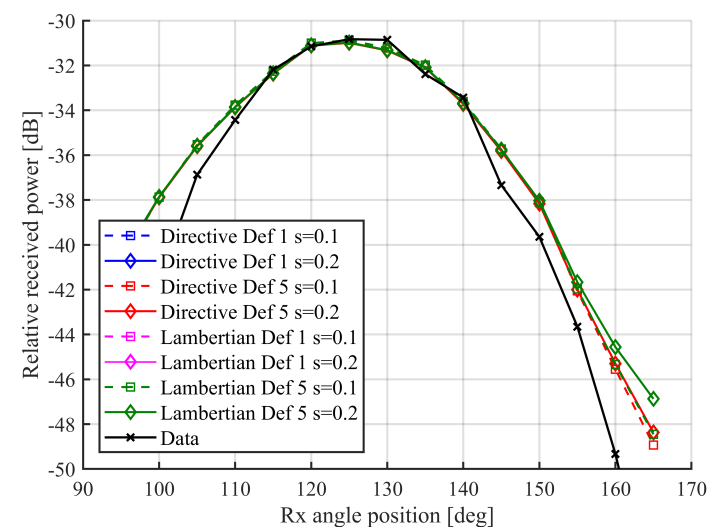

Figure 5.11: Ray-tracing results for metal @28 GHz, TX at 60 degrees

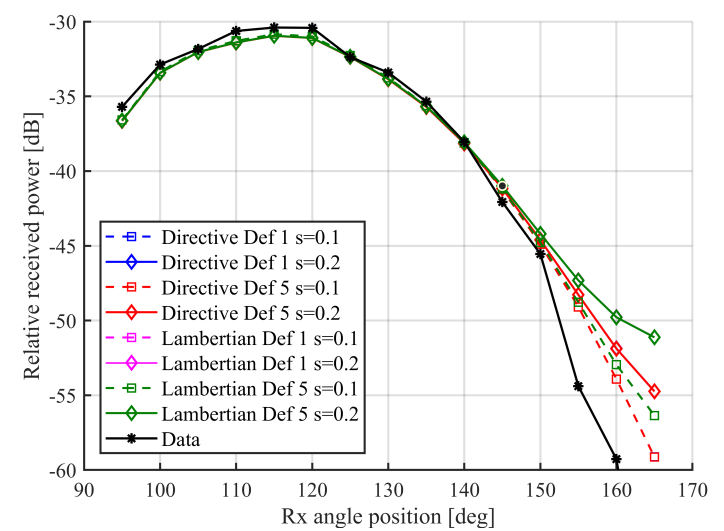

Figure 5.12: Ray-tracing results for metal @28 GHz, TX at 70 degrees 


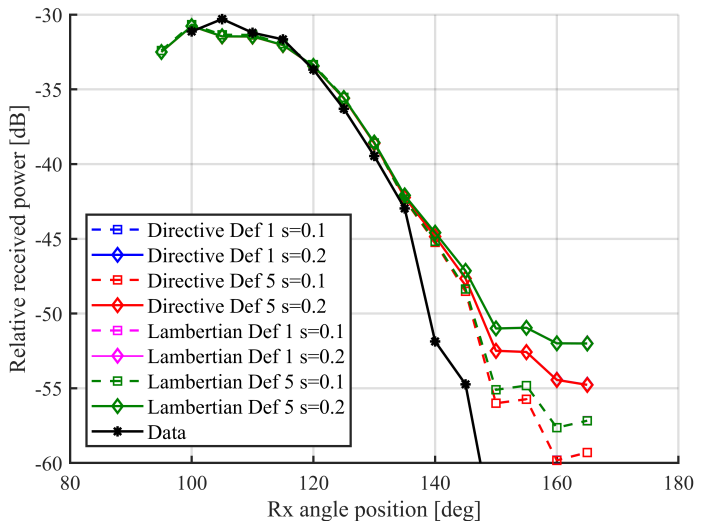

Figure 5.13: Ray-tracing results for metal @28 GHz, TX at 80 degrees

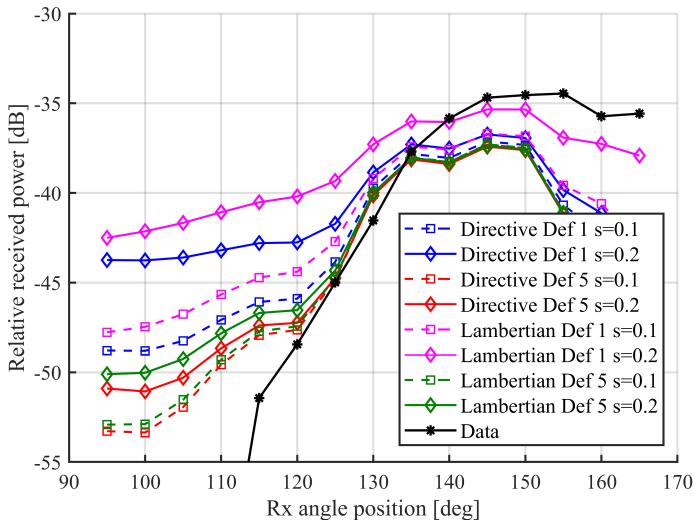

Figure 5.14: Ray-tracing results for concrete @28 GHz, TX at 30 degrees

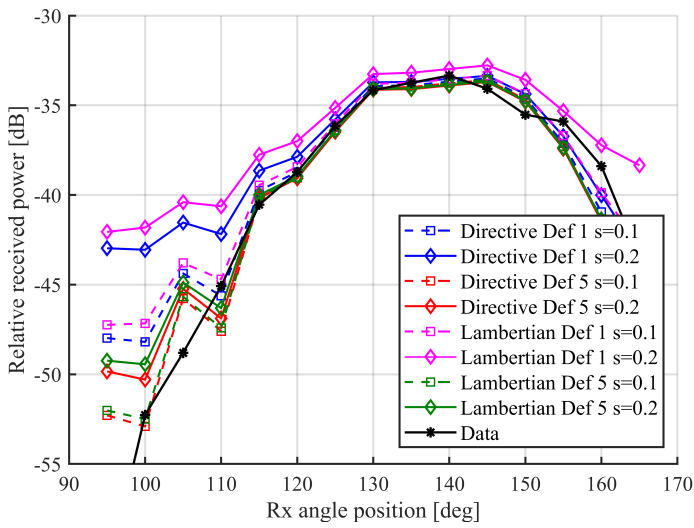

Figure 5.15: Ray-tracing results for concrete @28 GHz, TX at 40 degrees 


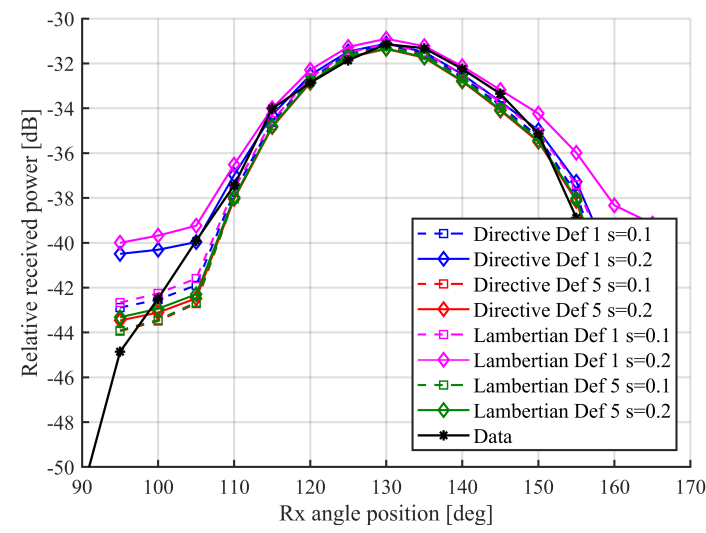

Figure 5.16: Ray-tracing results for concrete @28 GHz, TX at 50 degrees

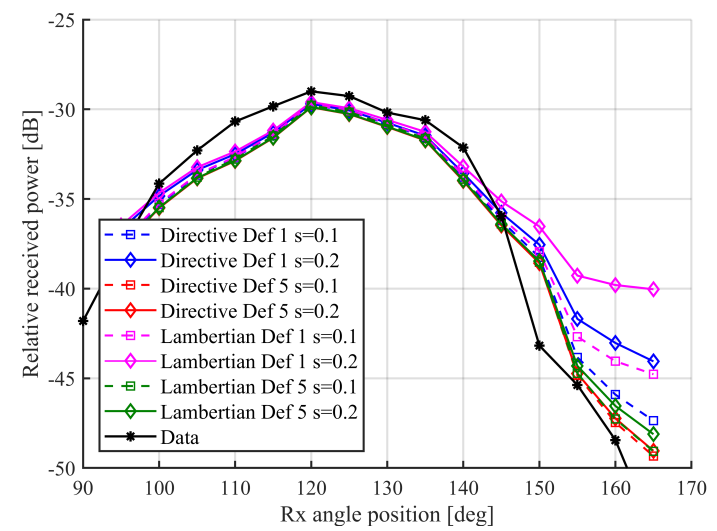

Figure 5.17: Ray-tracing results for concrete @28 GHz, TX at 60 degrees

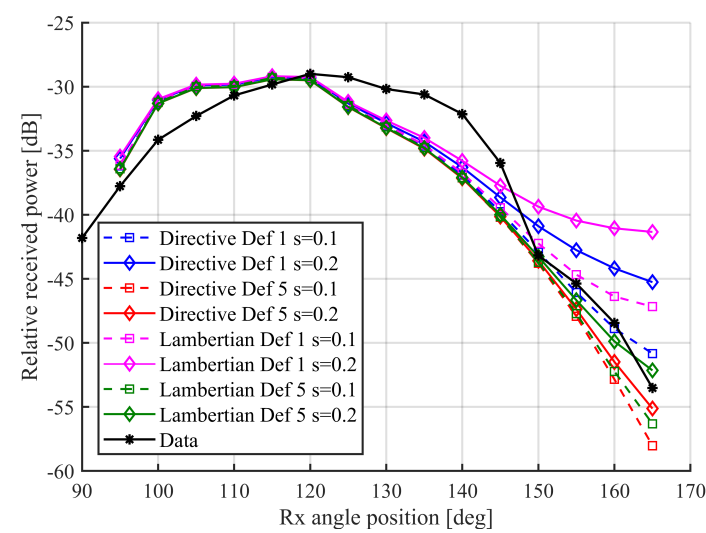

Figure 5.18: Ray-tracing results for concrete @28 GHz, TX at 70 degrees 


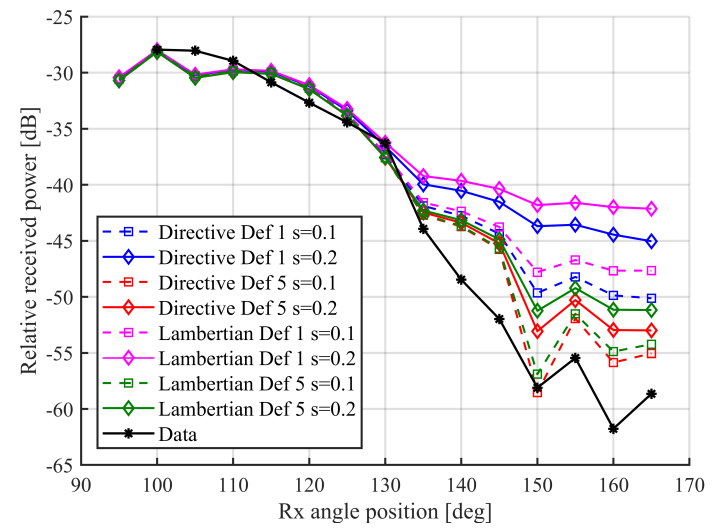

Figure 5.19: Ray-tracing results for concrete @28 GHz, TX at 80 degrees

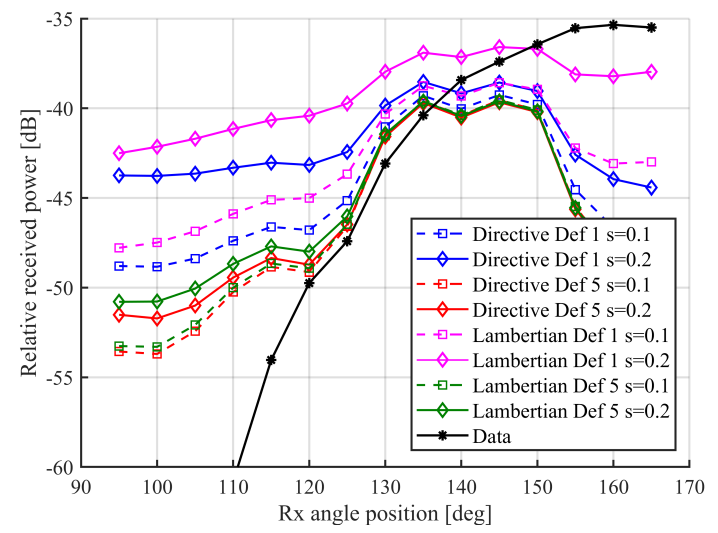

Figure 5.20: Ray-tracing results for chipboard @28 GHz, TX at 30 degrees

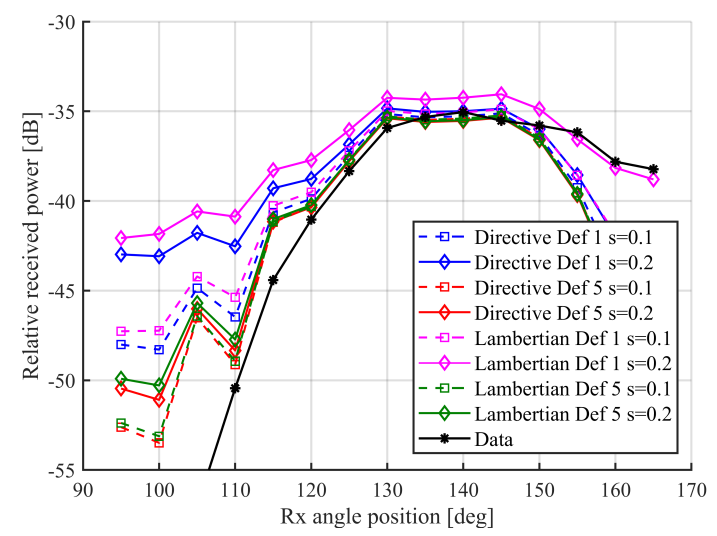

Figure 5.21: Ray-tracing results for chipboard @28 GHz, TX at 40 degrees 


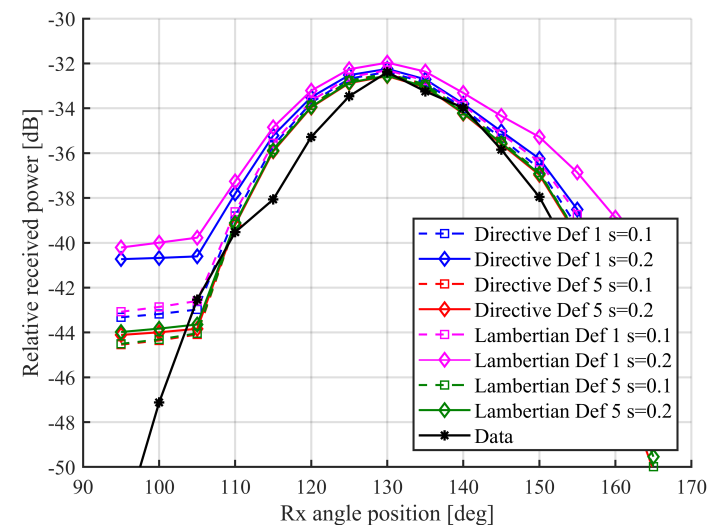

Figure 5.22: Ray-tracing results for chipboard @28 GHz, TX at 50 degrees

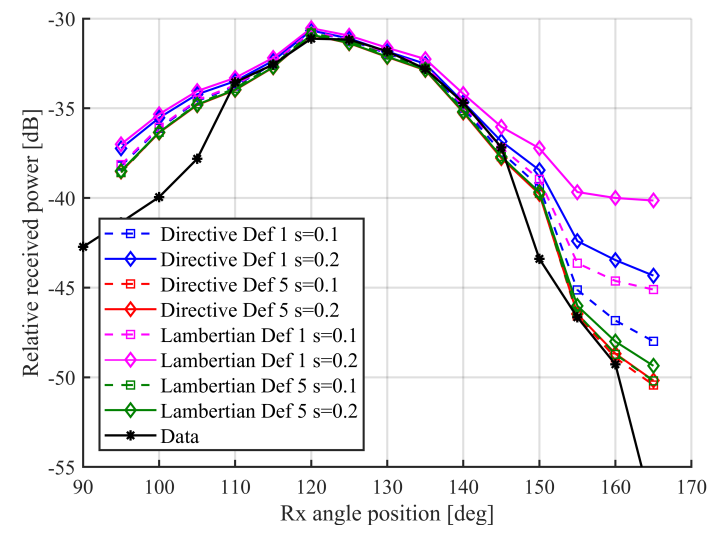

Figure 5.23: Ray-tracing results for chipboard @28 GHz, TX at 60 degrees

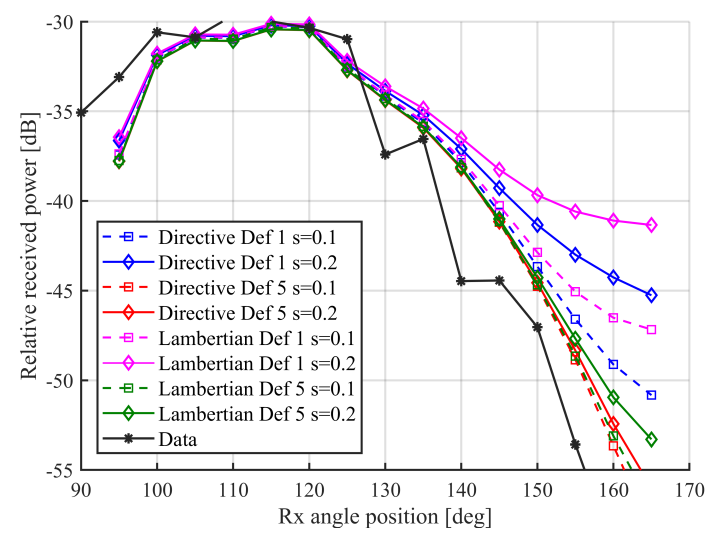

Figure 5.24: Ray-tracing results for chipboard @28 GHz, TX at 70 degrees 


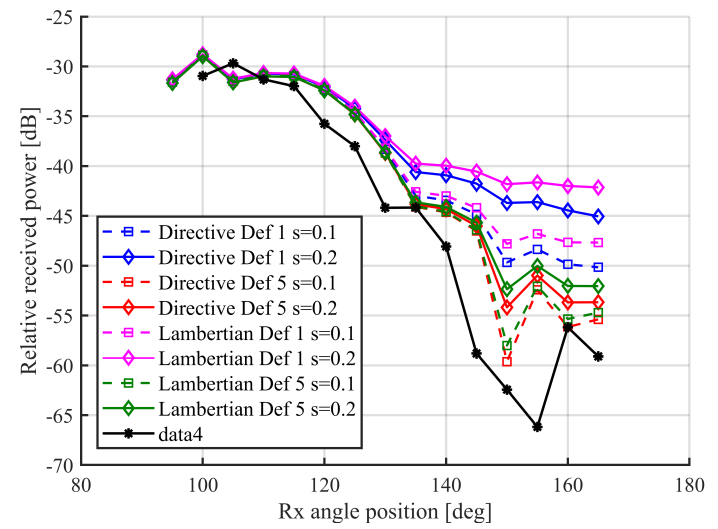

Figure 5.25: Ray-tracing results for chipboard @28 GHz, TX at 80 degrees

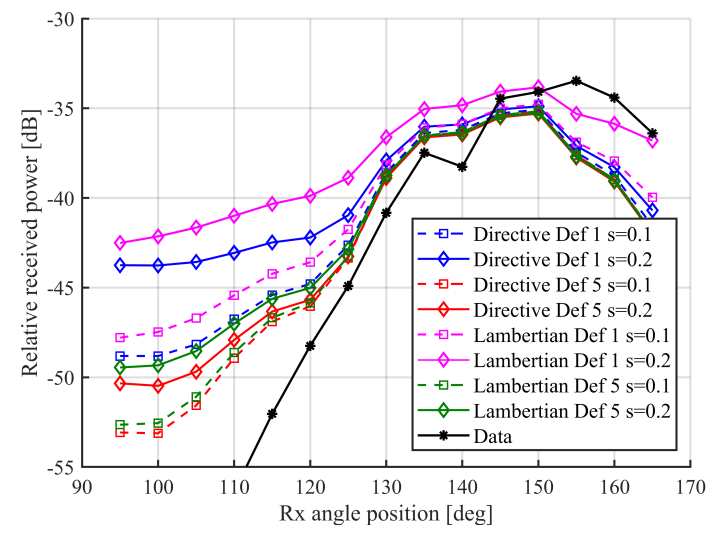

Figure 5.26: Ray-tracing results for tinted glass @28 GHz, TX at 30 degrees

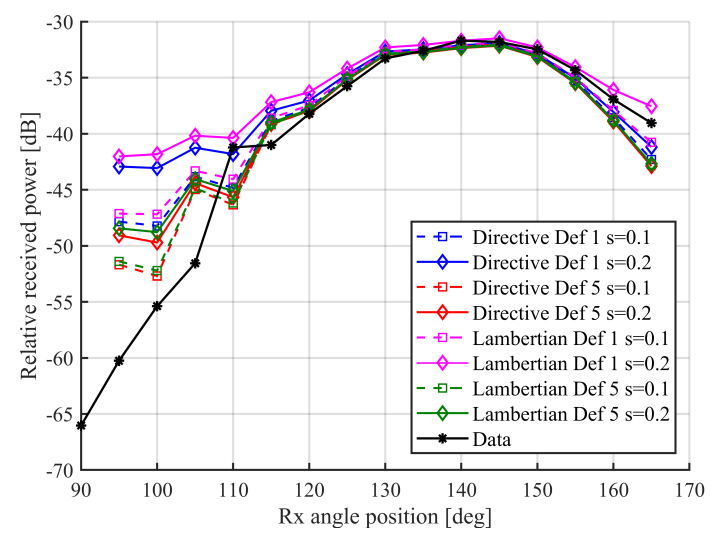

Figure 5.27: Ray-tracing results for tinted glass @28 GHz, TX at 40 degrees 


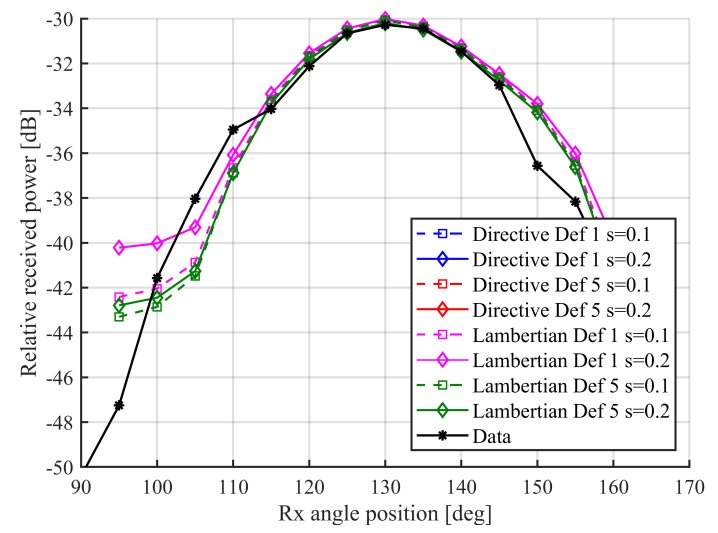

Figure 5.28: Ray-tracing results for tinted glass @28 GHz, TX at 50 degrees

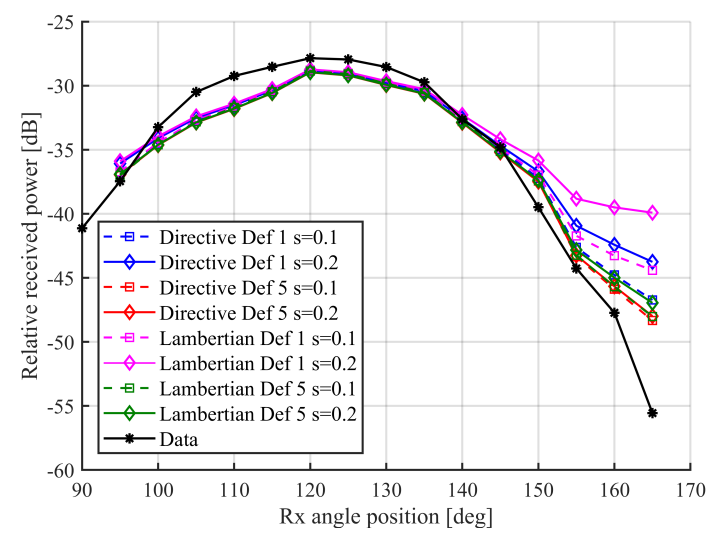

Figure 5.29: Ray-tracing results for tinted glass @28 GHz, TX at 60 degrees

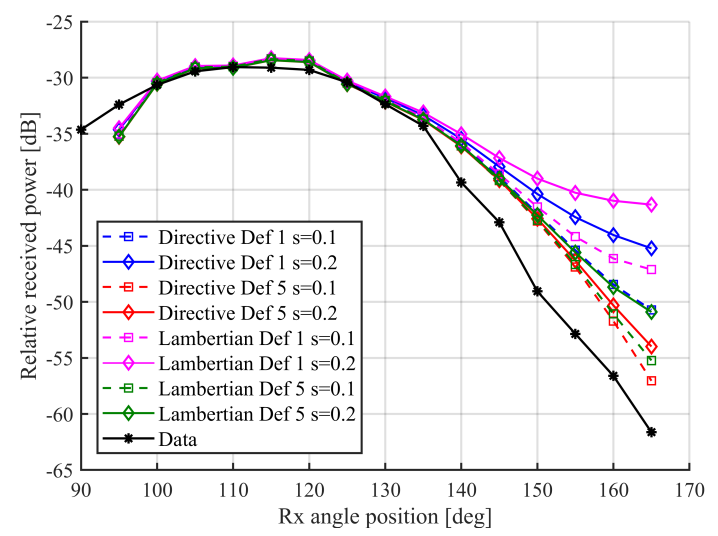

Figure 5.30: Ray-tracing results for tinted glass @28 GHz, TX at 70 degrees 


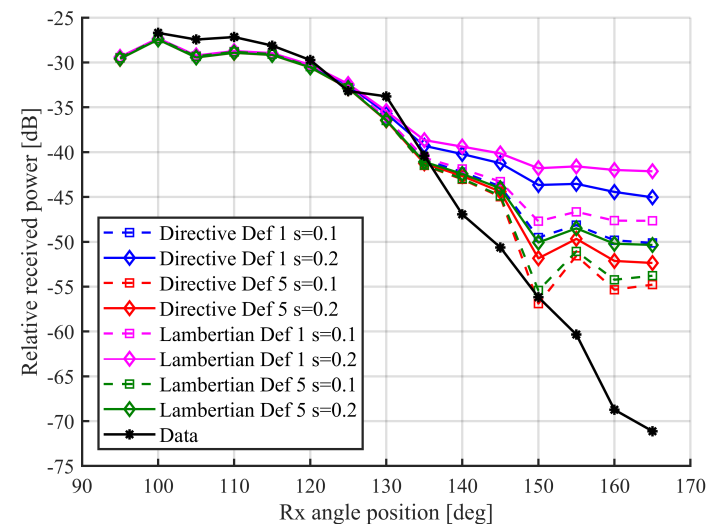

Figure 5.31: Ray-tracing results for tinted glass @28 GHz, TX at 80 degrees

\subsection{LIM Extension}

In Chapter 4, LIM has been used for the estimation of propagation losses in UMa and UMi scenarios. Although the predominant material in New York Univeristy (NYU) scenario is concrete, it is necessary to characterize other materials in order to extend the scope of LIM to other scenarios and improve its estimation accuracy. In this sense, this section describes the extension of LIM for the characterization of metal, concrete, chipboard and tinted glass. Special attention is given to model diffuse scattering, which is a significant component in radio propagation at $\mathrm{mmW}$.

Figure 5.32 shows the scenario implemented in Unity corresponding to the measurement campaign. The $9 \mathrm{RXs}$ have been placed in the semicircle from 90 to 170 degrees for the acquisition of light intensity. TX sweeps from 20 to 80 degrees to calculate all combinations of TX-RX. The TX is a spot light source, oriented to the center of the material with a conical aperture of 20 degrees and an intensity of 20 lux.

The geometrical detail of each material is taken into account for its characterization. For this purpose, a heights map has been designed with the typical normals of each material. Figure 5.33 shows the height map detail that contains information on the normals for each material. A completely smooth material will correspond to reflective materials such as metal and materials with the presence of roughness will behave as diffuse materials.

Another factor that has been considered is the rendering of the material. For this purpose, advanced shaders from Unity, High Definition Render 


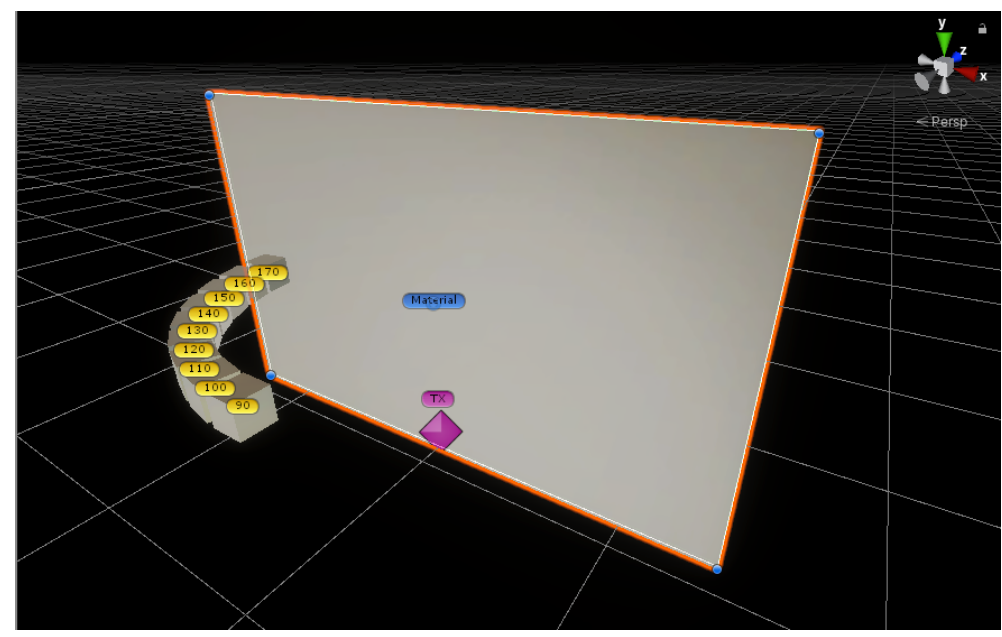

Figure 5.32: Scene view of diffuse scattering simulation in Unity

Pipeline (HDRP), have been used. HDRP has integrated variants of Bidirectional Reflectance Distribution Function (BRDF). For diffuse scattering the Lambertian model and the Oren Nayar model are integrated [116]. The Oren Nayar model is designed to model the diffuse scattering on rough surfaces. For smooth surfaces, the Lambertian model is enough according to [116]. In fact, in LIM tests, the results for both models are very similar, except for a slight difference in favor of Oren Nayar's model for concrete. This indicates that, for a future study of rougher materials, the Oren Nayar model should be considered. The Lambertian model for the BRDF can be expressed as:

$$
\rho^{\prime}\left(\theta_{i}, \phi_{i}, \theta_{r}, \phi_{r}\right)=\frac{\rho}{\pi},
$$

where $i$ and $r$ are the incident and reflected angles respectively, and $\rho$ is the reflectivity of the material. For the specular component, Ward's model has been considered. Ward's model adds a specular component to the Lambertian model and can be expressed as:

$$
\rho^{\prime}\left(\theta_{i}, \phi_{i}, \theta_{r}, \phi_{r}\right)=\frac{\rho_{d}}{\pi}+\frac{\rho_{s}}{\sqrt{\cos \theta_{i} \cos \theta_{r}}} \frac{e^{-\tan ^{2} \alpha / \sigma}}{4 \pi \sigma^{2}},
$$

where $\rho_{d}$ is the diffuse reflectance, $\rho_{s}$ is the specular reflectance, $\sigma$ is the root mean square of the surface roughness, and $\alpha$ is the reflected angle. At this point, it can be indicated that Ward's model is similar to the directive model proposed in [115]. In fact, the specular reflectance of the material would correspond to 


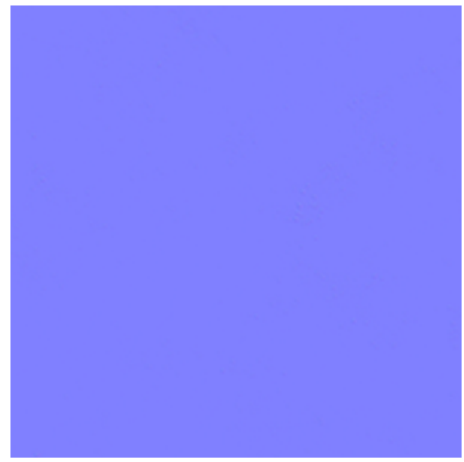

(a)

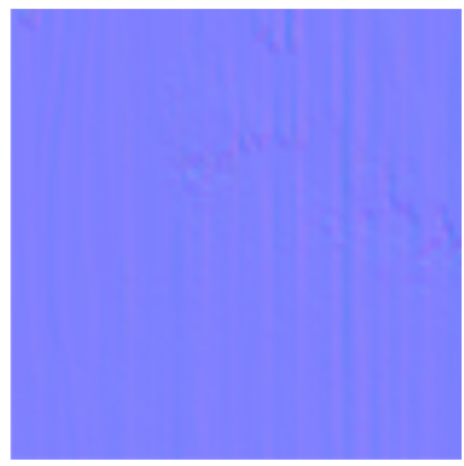

(c)

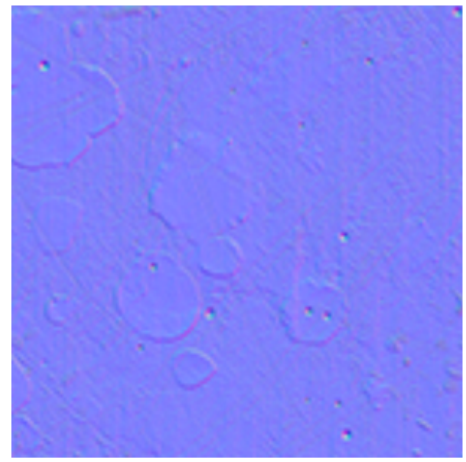

(b)

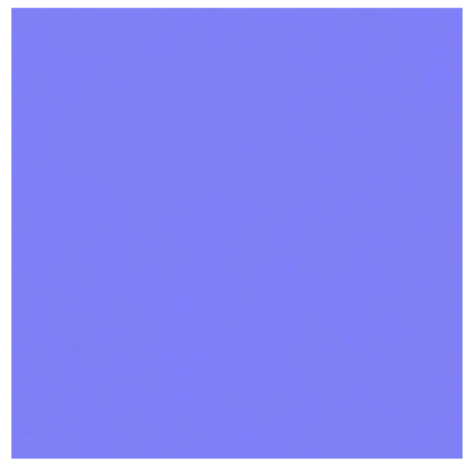

(d)

Figure 5.33: x50 zoom of normal height map for metal (a), concrete (b), chipboard (c) and tinted glass (d)

the reflection coefficient $\Gamma$ and the diffuse reflectance would be equivalent to the parameter $S$ of Lambertian and directive scattering models.

\subsubsection{Light Rendering Process}

The rendering process is where the indirect lighting on the scene is calculated. For each TX location, the indirect lighting has been calculated in pre-computed mode. The first step is to configure the materials of the objects in the scene. Figure 5.34 shows an example of material setup in Unity. Second, for each material all combinations of the BRDF variants have been tested. Third, lightmapping is set as shown in Figure 5.35. The baked light maps generation considers 32 direct samples, 512 indirect samples, 2 bounces, lightmap resolution is set 
to 40 texels per unit and the light map size set in $1024 \times 1024$ pixels. Lightmap is set to High Resolution. For this simple scenario, this setup and resolution of the lightmaps are adequate due to the small number of objects.

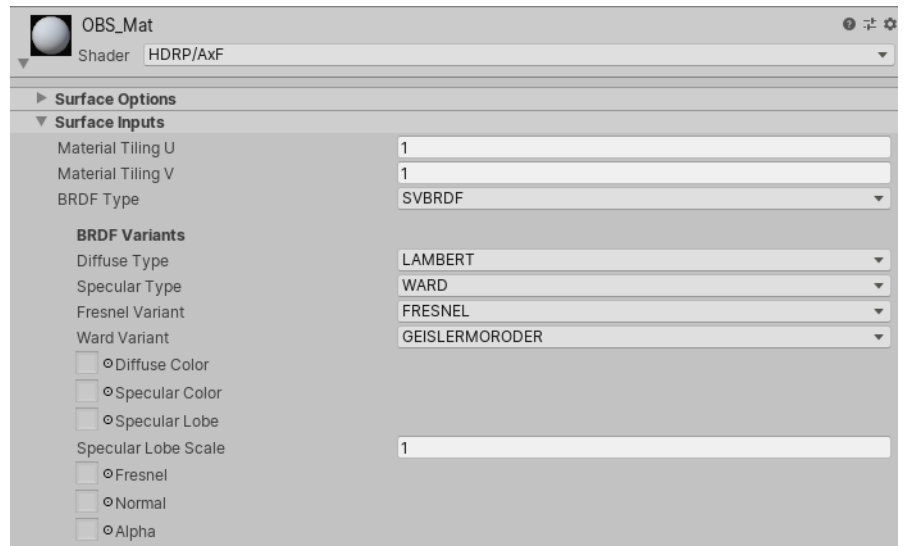

Figure 5.34: Material setup in Unity

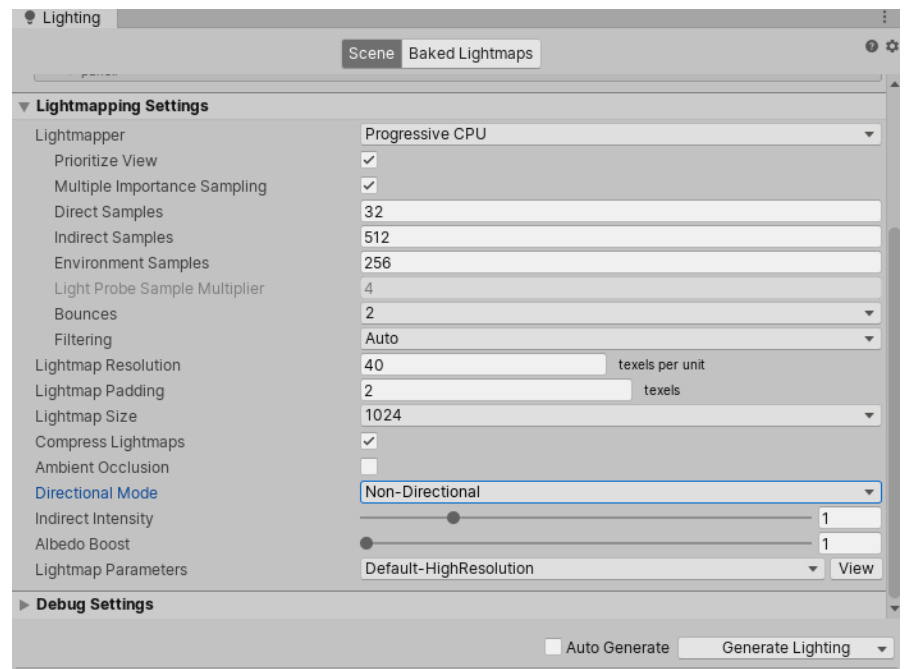

Figure 5.35: Lightmapping settings in Unity

For the calibration process, the Algorithm 1 shown in Chapter 4 has been followed. Lighting variables, Light Source Intensity (LSI), Light Bounce (LB), Shadow Strength (SS) and Shadow Bias (SB) were considered. Figure 5.36 


\section{CHAPTER 5. EXTENSION OF THE LIGHT INTENSITY}

MODEL

shows an example of a result of the pre-computed lighting process with diffuse scattering shaders.

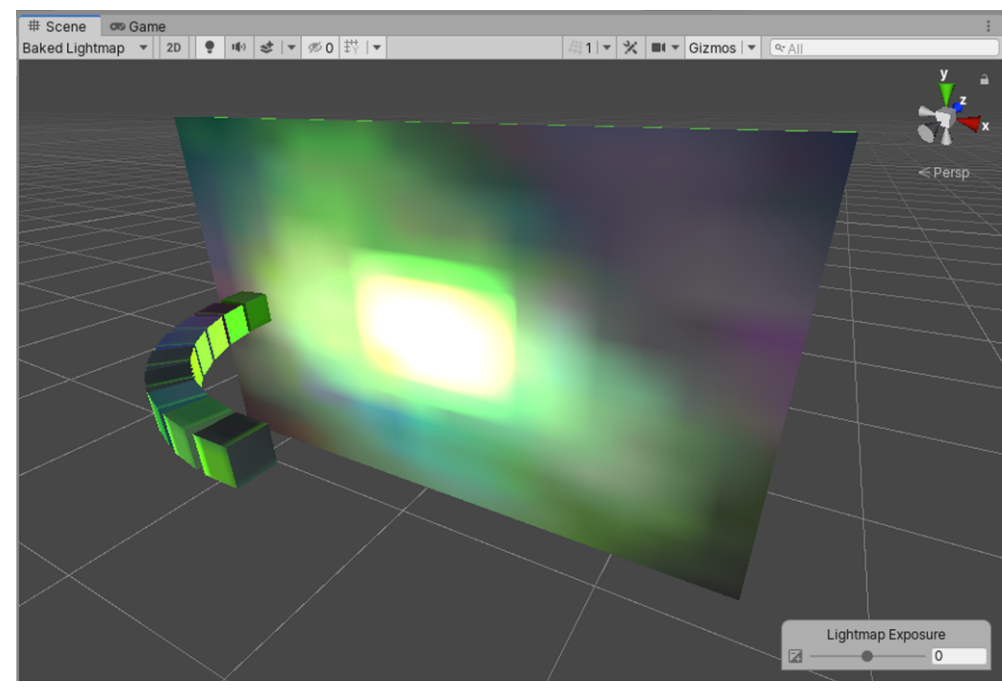

Figure 5.36: Backed light-map

\subsubsection{Results}

The lighting source variables that result from the above mentioned calibration process are shown in Table 5.3.

Table 5.3: Light source calibration variable values for $28 \mathrm{GHz}$

\begin{tabular}{|c|c|}
\hline Lighting variables & $\mathbf{2 8} \mathbf{~ G H z}$ \\
\hline Light source intensity (LSI) & 20 lux \\
\hline Light bounce (LB) & 0.35 \\
\hline Shadow strength (SS) & 0.55 \\
\hline Shadow bias (SB) & 0.90 \\
\hline
\end{tabular}

After having tested all combinations of Unity's BRDF variants, the rendering settings for each material are summarized in the Table 5.4.

Following the same LIM calibration process presented in Chapter 4, relative received power of measurement and light intensity values acquired from LIM 
Table 5.4: Shader setup

\begin{tabular}{|l|l|l|l|l|}
\hline Material & $\begin{array}{l}\text { BRDR } \\
\text { Type }\end{array}$ & $\begin{array}{l}\text { Diffuse } \\
\text { Type }\end{array}$ & $\begin{array}{l}\text { Specular } \\
\text { Type }\end{array}$ & $\begin{array}{l}\text { Fresnel } \\
\text { Variant }\end{array}$ \\
\hline Metal & SVBRDR & Lambertian & Ward & Fresnel \\
\hline Concrete & SVBRDR & Oren Nayar & Ward & Fresnel \\
\hline Chipboard & SVBRDR & Lambertian & Ward & Fresnel \\
\hline Tinted glass & SVBRDR & Lambertian & Ward & Fresnel \\
\hline
\end{tabular}

were approximated with polynomial correlation function. The best polynomial adjustment at $28 \mathrm{GHz}$ is the following:

$$
\operatorname{RRP}[d B]=119.8 i^{2}-18.1 i-105.2,
$$

where RRP is the relative received power and $i$ is the normalized light intensity value.

Figure 5.37 shows the scatter plot of the relative received power and the normalized light intensity values for the measurement data points at $28 \mathrm{GHz}$ for metal, concrete, tinted glass and chipboard materials. The figure also shows the polynomial adjustment curve under consideration.

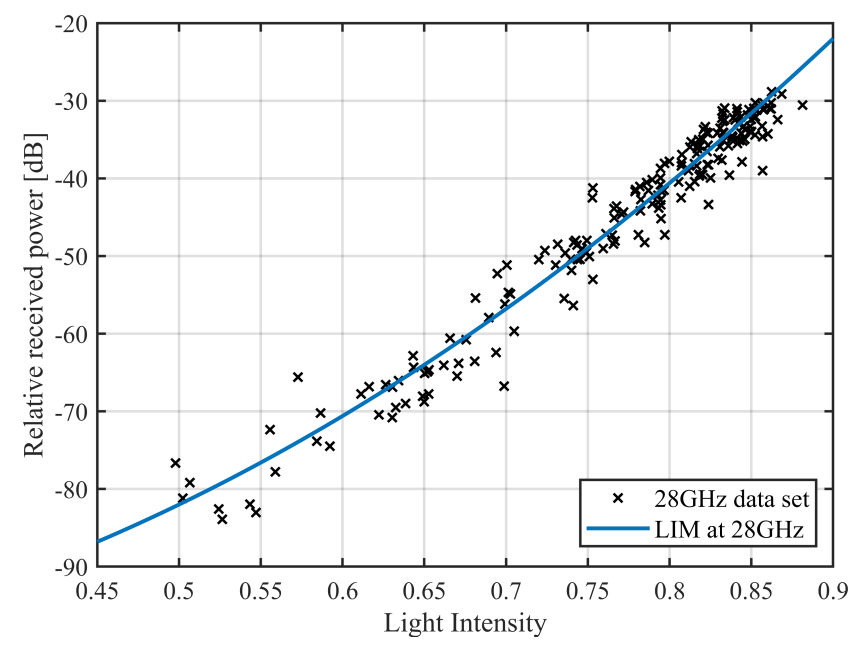

Figure 5.37: Scatter plot of relative received power and light intensity correlation for $28 \mathrm{GHz}$ and LIM polinomial adjustments 
In order to validate the polynomial calibration, the coefficient of determination or R-square $R^{2}$ and the RMSE of the estimate were used. A value of $R^{2}$ of 0.83 was obtained. LIM obtained RMSE values of $3.32,3.57,3.35$ and $3.33 \mathrm{~dB}$ for metal, concrete, chipboard and tinted glass, respectively. The predictive accuracy of the extension of LIM is better than LIM without the characterization of materials.

Figure 5.38 shows the Cumulative Distribution Function (CDF) of LIM and ray-tracing estimation. It can be clearly seen that ray-tracing estimates are more accurate, and the probability of estimation error is lower than LIM. However, it can be indicated that this difference has been reduced, with respect to the LIM model presented in the Chapter 4. This higher accuracy comes with an increased computational complexity although, as it will be shown in Section 5.4.3, LIM computational demands are still significantly lower than those of ray-tracing.

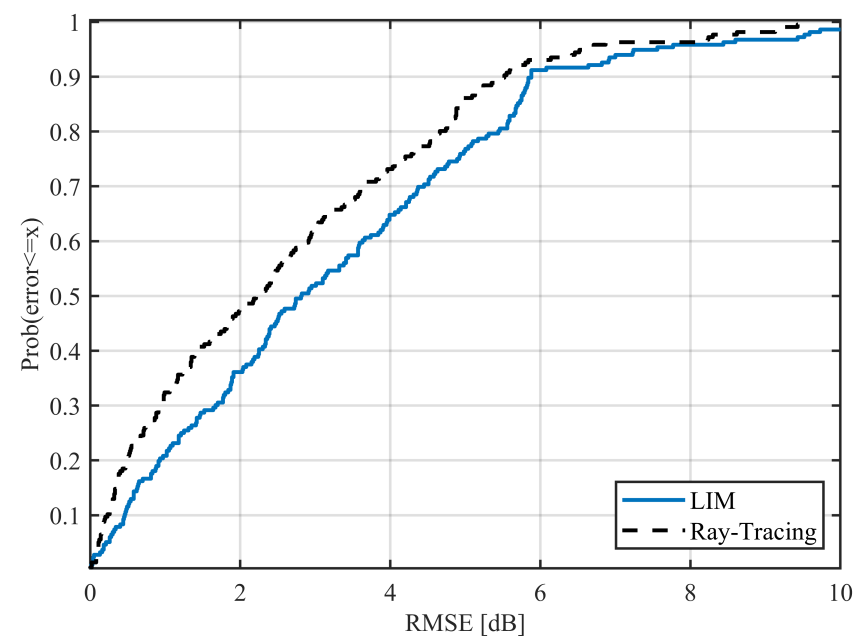

Figure 5.38: CDF of RMSE for LIM and ray-tracing at $28 \mathrm{GHz}$

Figures 5.39 to 5.44 show the measurements of the experiment and the relative received power estimate of LIM for TX positions from 30 to 80 degrees. A better adjustment can be observed at positions where specular components are most significant. The results obtained are satisfactory, with less than $3.6 \mathrm{~dB}$ of RMSE for all the materials. 


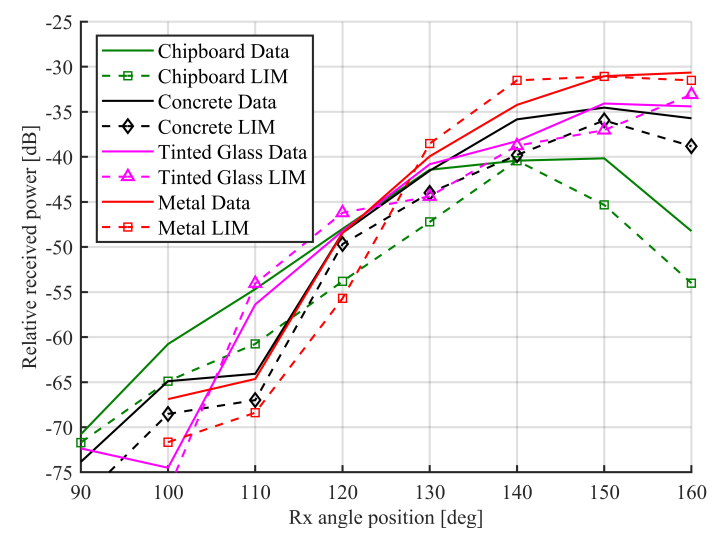

Figure 5.39: LIM results for $28 \mathrm{GHz}$. TX at 30 degrees

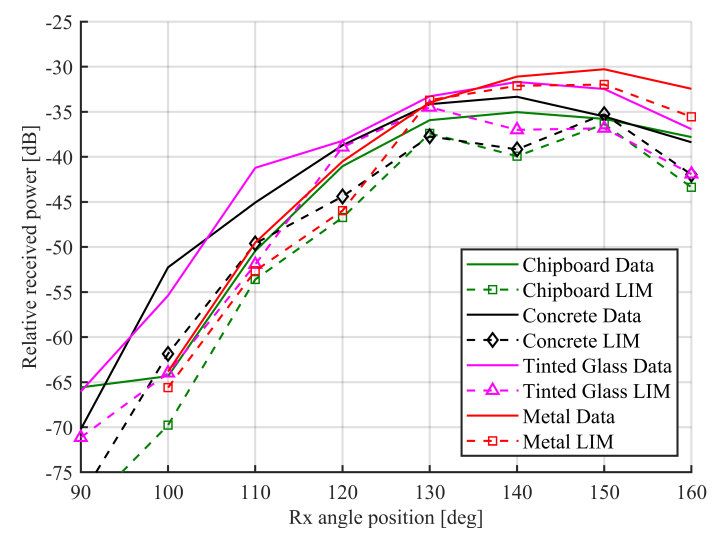

Figure 5.40: LIM results for $28 \mathrm{GHz}$. TX at 40 degrees

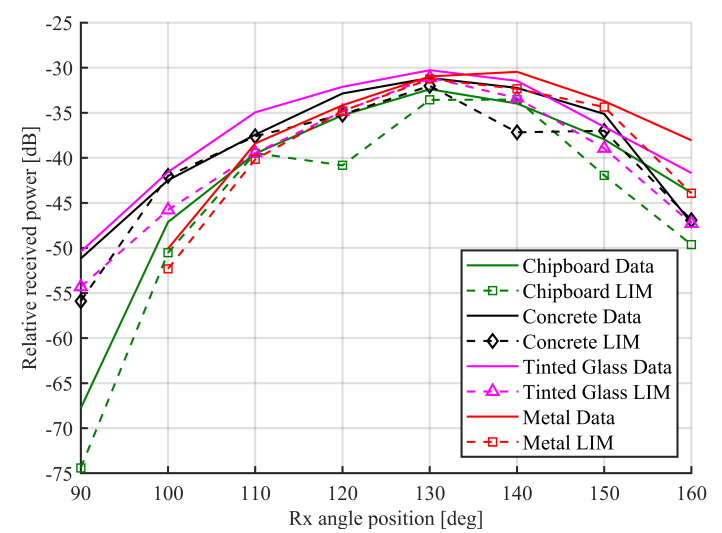

Figure 5.41: LIM results for $28 \mathrm{GHz}$. TX at 50 degrees 


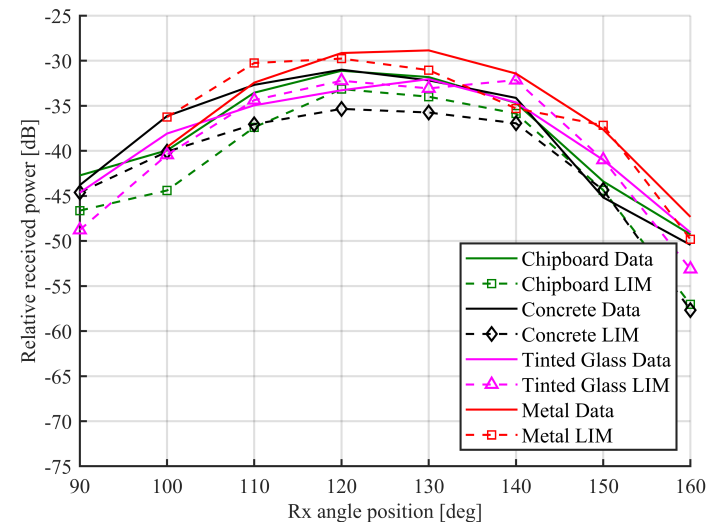

Figure 5.42: LIM results for $28 \mathrm{GHz}$. TX at 60 degrees

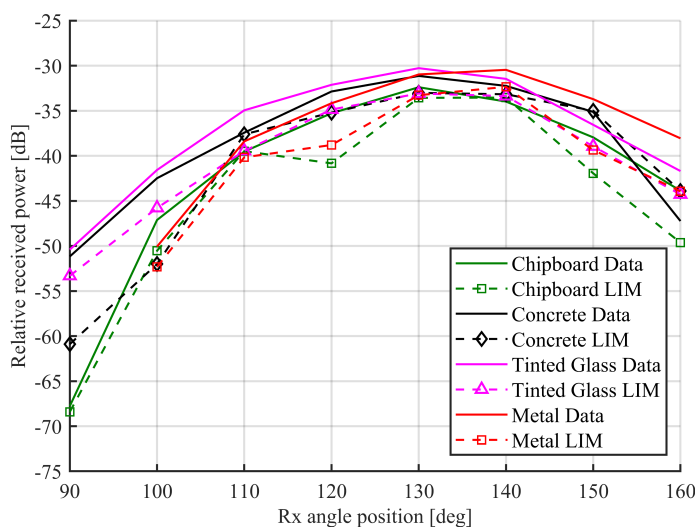

Figure 5.43: LIM results for $28 \mathrm{GHz}$. TX at 70 degrees

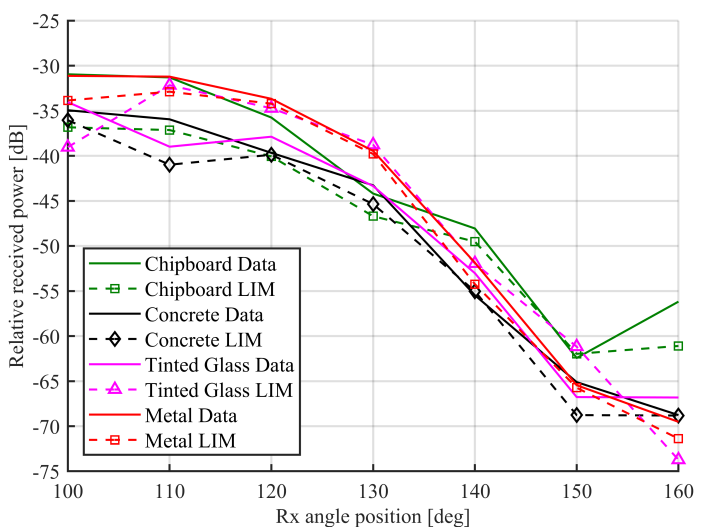

Figure 5.44: LIM results for $28 \mathrm{GHz}$. TX at 80 degrees 


\subsubsection{Computational Complexity}

Computational complexity has been evaluated following the same procedure used in chapter 4 . In that sense, this section compares LIM in terms of use of computing resources with a ray-tracing tool. In this experiment, the same virtual scenario is used and we repeat the measurement campaign as we did with LIM. The metric of evaluation was the average number of F-flops that LIM and ray-tracing needed to calculate the large-scale loss of a single measurement point. The flops were calculated as

$$
F=t_{\text {algorithm }} \cdot\left(G F L O P S_{\text {core }} \cdot N_{\text {cores }} \cdot C P U_{\text {usage }}\right),
$$

where $t_{\text {algorithm }}$ is the time that it takes for the algorithm to perform the calculation in seconds, GFLOP $S_{\text {core }}$ is the average use of the Central Processing Unit (CPU) while the algorithm is running. The calculation process was performed on a computer equipped with an Inter(R) Core (TM) i7-4720HQ processor that runs 8 cores in parallel (Ncores $=8$ ) at $2.60 \mathrm{GHz}$ each, with GFLOP Score $=3.51$. The resulting number of flops, and all other parameters in 5.27, for ray-tracing and LIM are shown in Table 5.5. As expected, LIM is significantly faster than ray-tracing and shows one orders of magnitude less flops than ray-tracing.

Table 5.5: Calibration variable values for $28 \mathrm{GHz}$

\begin{tabular}{|l|l|l|}
\hline Parameter & Ray-tracing & LIM \\
\hline$t_{\text {algorithm }}$ & 161.40 & 13.01 \\
\hline$C P U_{\text {usage }}$ & 0.13 & 0.11 \\
\hline Flops $(\mathrm{F})$ & $7.61 \cdot 10^{9}$ & $2.92 \cdot 10^{8}$ \\
\hline RMSE $[\mathrm{dB}] 28 \mathrm{GHz}$ & 2.80 & 3.39 \\
\hline
\end{tabular}

\subsection{Conclusions}

In this chapter, an extension of LIM for diffuse scattering modeling and material characterization has been presented in order to increase the accuracy in the estimation of propagation losses for $\mathrm{mmW}$. The results obtained with LIM are very similar to the ray-tracing but with a substantially lower computational 
complexity. For the LIM extension, four materials have been characterized (metal, concrete, chipboard and tinted glass) obtaining favorable results. To further improve the LIM model it is necessary to perform measurement campaigns with other materials that will probably decrease the error estimation of the model. This modeling will allow the use of LIM in more complex scenarios, such as indoor, where there is a great diversity of materials. First, in order to properly exploit Unity's graphics engines, it is necessary to use Graphics Processing Units (GPUs) with better performance than in the first place, it will decrease rendering time and lighting. And, secondly, it will improve the precision of the rendering and illumination of the scenes that will translate into a decrease in the estimation error. Finally, it can be indicated that the use of LIM can be considered a strong candidate for the characterization of the radio channel in highly complex scenarios. 


\section{Chapter 6}

\section{Light Intensity Model Evaluation in Indoor Scenarios at $28 \mathrm{GHz}$}

In this chapter, the Light Intensity Model (LIM) approach is applied to estimate the path loss in the well-known indoor scenario of the New York Univeristy (NYU), where measurements and path loss models at $28 \mathrm{GHz}$ and $73 \mathrm{GHz}$ are available [51].

The remainder of the chapter is as follows:

- Section 6.1 explains the importance of modeling the propagation of Millimeter Wave $(\mathrm{mmW})$ in indoor scenarios and the scope of the assessment in this chapter.

- Section 6.2 introduces the path loss models used by the Third Generation Partnership Project (3GPP) and NYU channel models.

- Section 6.3 describes the new modeling proposed in this chapter.

- Section 6.4 details the characteristics of the indoor scenario of the NYU used in the assessment, and explains how it has been modeled in the Three Dimensions (3D) game engine.

- Section 6.5 analyzes the accuracy of the LIM model based on the comparison with real measurements, and the predictions of stochastic models.

- Section 6.6 draws the main conclusions of the chapter. 


\subsection{Introduction}

The Fifth Generation (5G) of mobile and wireless communications promises to be a technological revolution which requires new enabling technologies to meet its challenging goals. One of these enablers for $5 \mathrm{G}$ is the use of $\mathrm{mmW}$ bands. As indicated in the state of the art, the use of millimeter bands will help to achieve the capacity and latency requirements of the $5 \mathrm{G}$ due to the enormous bandwidths available. However, the high path loss inherent to $\mathrm{mmW}$ due to propagation and obstructions represents a great challenge. In previous chapters, the study of propagation losses has focused on outdoor scenarios. However, it is foreseen that $\mathrm{mmW}$ will be also exploited in indoor scenarios through the deployment of small cells. These cells would reuse spectrum locally and would provide most of the capacity, above all indoors, while macro-cells would provide wide coverage and less capacity [117]. The high losses due to obstructions is not a great problem for small cells in indoor due to its short range, while it is clearly positive since it reduces the interference between adjacent cells.

Therefore, given the importance of $\mathrm{mmW}$ in indoor for $5 \mathrm{G}$, in this chapter the Thesis focuses on the application of LIM to an indoor scenario. In order to properly evaluate the performance of LIM, an office scenario has been chosen in which measurements at $28 \mathrm{GHz}$ are available together with information about the distribution and type of materials that compose the scenario. In this specific scenario, the configuration of the materials carried out in the previous chapter has been implemented to emulate properly the diffuse scattering of the materials. To validate the model, a comparison between LIM and various stochastic methods has been performed, as a first step to determine the validity of LIM in these scenarios, leaving out of this comparison other ray-tracing implementations.

\subsection{Stochastic Channel Models for $\mathrm{mmW}$ in 5G}

The high path loss and severe fading due to obstructions inherent to the $\mathrm{mmW}$ band are challenging for successful deployment of $5 \mathrm{G}$ systems. In this context, an accurate channel modeling becomes crucial.

A simple modeling is provided by the stochastic channel models. They are based on probability density functions to characterize some parameters of the channel, being this functions different for each environment. Stochastic models can take into account the geometry of the scenario, mainly the relative position and orientation of the transmitters an receivers. Concerning the propagation losses modeling, they usually have probability functions to determine if the receiver and the transmitter are in line of sight or not, and for each kind of 
sight, they provide simple functions to calculate the losses. The models of the WINNER II project [118], the IMT-2020 models [39] and the 3GPP models [37] are relevant examples.

Particularly, focusing on indoor scenarios, the 3GPP model is described mathematically as follows:

$$
\begin{gathered}
P L_{L o S}[d B]=32.4+17.3 \log _{10}\left(d_{3 D}\right)+20 \log _{10}\left(f_{c}\right) \\
P L_{N L o S}^{\prime}[d B]=38.3 \log _{10}\left(d_{3 D}\right)+17.3+24.9 \log _{10}\left(f_{c}\right), \\
P L_{N L o S}[d B]=\max \left(P L_{L o S}, P L_{N L o S}^{\prime}\right),
\end{gathered}
$$

where $P L_{L o S}[d B]$ is the path loss in case of Line of Sight (LoS), $P L_{N L o S}[d B]$ is the path loss in case of Non Line of Sight (NLoS), $f_{c}$ is the frequency in $\mathrm{GHz}$, and $d_{3 D}$ is the $3 \mathrm{D}$ distance between the transmitter and the receiver. In this work, a quasi-deterministic path loss model based on 3GPP model will be evaluated. For the path loss computation, (6.1), (6.2) and (6.3) provided by the $3 \mathrm{GPP}$ will be used. However, whether the receivers are in LoS or not, will be determined by using deterministic methods.

NYU Wireless research group has been very active in the field of channel modeling for $5 \mathrm{G}$ and millimeter waves. In this context, NYU has carried out measurement campaigns for both indoor and outdoor scenarios. In [51], the authors propose single frequency and multi-frequency path loss models based on measurements from an indoor environment at $\mathrm{mmW}$ as shown in Figure 6.1. Directional and omnidirectional path loss models are obtained considering 3D distance between transmitters and receivers. Measurements derived from co-polarized and cross-polarized path loss model are provided. This dataset encourages a comparative study, serving as a stochastic model reference. Concretely, the single-frequency omnidirectional close-in free space reference distance (CI) path loss model provided by [51] has been selected for the purpose of this work. Regarding polarization, it is also worth to mention that only vertical-vertical co-polarized transmissions are in the scope of this study. For the large-scale propagation loss estimation it proposes the following equation for both LoS and NLoS:

$$
P L^{C I}(f, d)[d B]=F S P L\left(f, d_{0}\right)+10 n \log _{10}\left(\frac{d}{d_{0}}\right)+X_{\sigma}^{C I},
$$

for $d \geq d_{0}$ with $d_{0}=1 \mathrm{~m}$, where FSPL is the free space path loss, $d_{0}$ is the reference distance, $n$ is the Path Loss Exponent (PLE) and, $X_{\sigma}^{C I}$ is the scattering factor that represents large-scale signal fluctuations as a result of shadows due to wireless channel obstructions and is defined as a zero-Gaussian 


\section{CHAPTER 6. LIGHT INTENSITY MODEL EVALUATION IN INDOOR SCENARIOS AT 28 GHZ}

random variable $\sigma$ in $\mathrm{dB}$. However, in our case, $X_{\sigma}^{C I}$ has been disregarded since the propagation conditions are known.

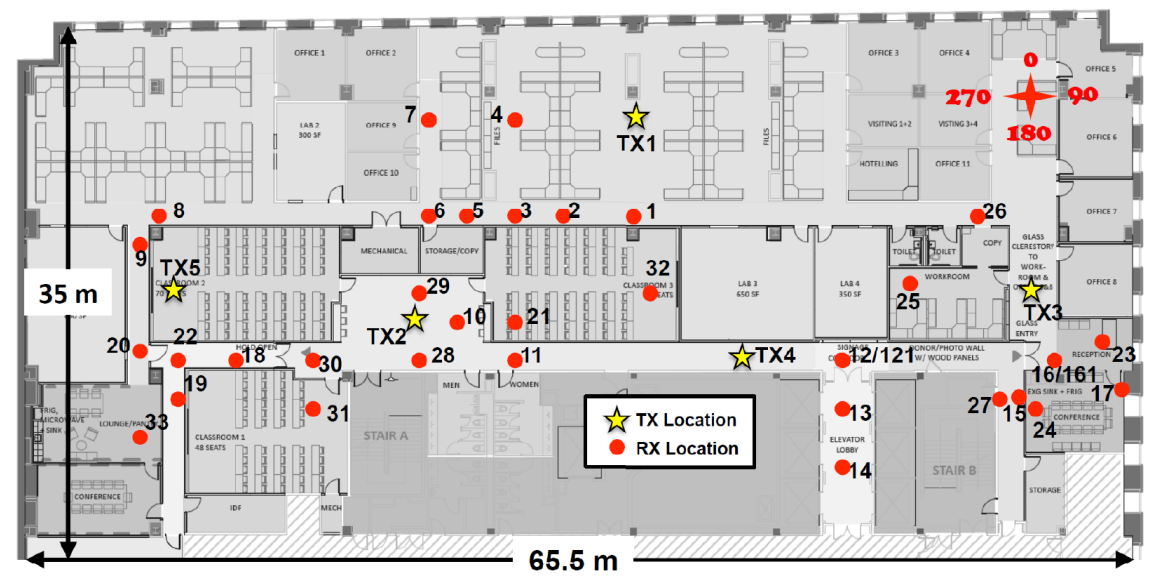

Figure 6.1: Map of the MetroTech Center 9th floor with five TX locations and $33 \mathrm{RX}$ locations. The yellow stars represent the TX locations and the red dots represent the RX locations [83]

In this work, both $3 \mathrm{GPP}$ based quasi-deterministic model and CI model from [51] will be compared to LIM.

\subsection{Light Intensity Channel Model}

Given the spectral closeness between the visible spectrum and the mmW bands, it seems appropriate to propose the use of light propagation modeling tools for radio channel modeling. Considering this, LIM comes up as a model for estimating large-scale propagation losses in $\mathrm{mmW}$ based on the light modeling of game engines. LIM replaces the signal transmitters with light sources, then light propagates through the scenario and finally the received light intensity at each specific point is translated into propagation losses via a simple polynomial function.

LIM exploits the two most important resources of game engines: the first one, a great capacity and friendly developer interface that facilitate the creation process of super realistic scenarios. And the second, the advanced ray-tracing algorithms of the lighting processes performed by the game engines. The ability to create realistic scenarios is of paramount importance to emulate in detail the physical phenomena as they happen in reality due to the specific geometry of 
the real scenarios. Concerning the lighting processes, game engines have specific tools for those tasks. For example, Unity has the Global Illumination (GI) advanced lighting tool. The GI is a group of algorithms and mathematical models that attempts to simulate the complex behavior of light, i.e. specular reflections, diffuse reflections, refractions and each one of the phenomena associated with the propagation of light.

Another relevant feature of Unity is that it is possible to modify the configuration of the materials of the $3 \mathrm{D}$ objects to have an interaction with the light closer to the interaction of those materials with the radio waves. In the case of our LIM implementation, the texture of the materials is adjusted with that aim. Specifically, we use extremely smooth materials to emulate high reflective materials such as metal while different levels of roughness are assigned to materials originating diffuse scattering.

\subsubsection{Acquisition of Light Intensity Values}

As explained, LIM associates a light intensity value received at a certain point of an scenario with a propagation loss. For the acquisition of light intensity, cameras are located at the point of interest. In order to capture light from all directions of the scene, not a single camera is used. Instead, a $0.05 \mathrm{~m}$ side cube is placed at the Rx position, and the intensity of the light is obtained by assigning and focusing a camera to each and every one of the 6 faces of the cube to capture the light intensity at each plane. The total light intensity of the $\mathrm{Rx}$ is the sum of the light intensity of the 6 faces of the cube. The position of the cameras is not a problem because the cameras are completely transparent and do not create shadows by themselves. The light intensity for each cube side is normalized by dividing the intensity measured by the maximum value achievable if the source was co-located with the cube side. As a result, the total intensity is in the range $[0,6]$.

\subsubsection{Global Illumination and Light Rendering Process}

The two main components of GI are light sources and High Definition Render Pipeline (HDRP). The four main parameters of the light sources are the Light Source Intensity (LSI), the Light Bounce (LB), the Shadow Strength (SS) and the Shadow Bias (SB) [94]. These four characteristics determine the light intensity values in the scenario. Briefly, LSI sets the brightness of diffuse ambient light in the scene. LB is used to set how many reflection bounces are allowed. SS and SB determine how dark the shadows are and adjust the position and definition of the shadows, respectively. Concerning to HDRP, it is an advanced shader capable of modeling the response of direct and indirect light in real 
time, with multiple Bidirectional Reflectance Distribution Functions (BRDFs) [94]. The BRDFs available in Unity for specular reflection are the Ward model and the Phong model. Both models add a specular component, the first as a function of the normal of the reflection point and the second as a function of the normal of the surface of the object. For diffuse scattering, the Oren Nayar model and the Lambertian model are available. The Oren Nayar model is designed to model scattering on rough surfaces. For smooth surfaces, the Lambertian model is sufficient according to [116]. For the Unity calibration, all possible combinations between the diffuse and reflective functions that best match the electromagnetic behavior of the materials have been evaluated.

\subsubsection{Light Intensity Model Calibration}

Transmitters and receivers were placed in the synthetic scenario strictly following the location coordinates and the height and orientation specifications in the actual measurement campaign as shown in Figure 6.2. Omnidirectional light sources were placed one by one at transmitter locations and light intensity values were collected at receiving points.

For the calibration process, all combinations of the five above mentioned Unity's lighting variables were tested. For each combination, a light intensity is obtained at each measurement point. Then, it is find the polynomial function that returns path loss values from the light intensities such as the mean absolute error between the predicted path loss and the measured path loss is minimized. Once the polynomials and their mean absolute errors are available for all the lighting variables combinations, it is selected the polynomial and combination which produces the lowest error.

\subsection{Evaluation Scenario and Unity Configura- tion}

Using Unity, the real indoor scenario of NYU Wireless research center has been modeled as shown in Figure 6.2. This scenario comprises spaces such as common cubicle offices, lecture rooms and corridors, in which different propagation characteristics are manifested. TX and RX positions are marked in Figure 6.2. It is necessary to indicate that since in this work neither transmission nor diffraction have been considered, some Tx-Rx measurements in [51] have been discarded.

In Unity 3D, it is possible to characterize the interaction of each material with the light in a different way. Specifically, through the GI process, specular and diffuse reflection models of BRDF that best fit the reflective behavior 


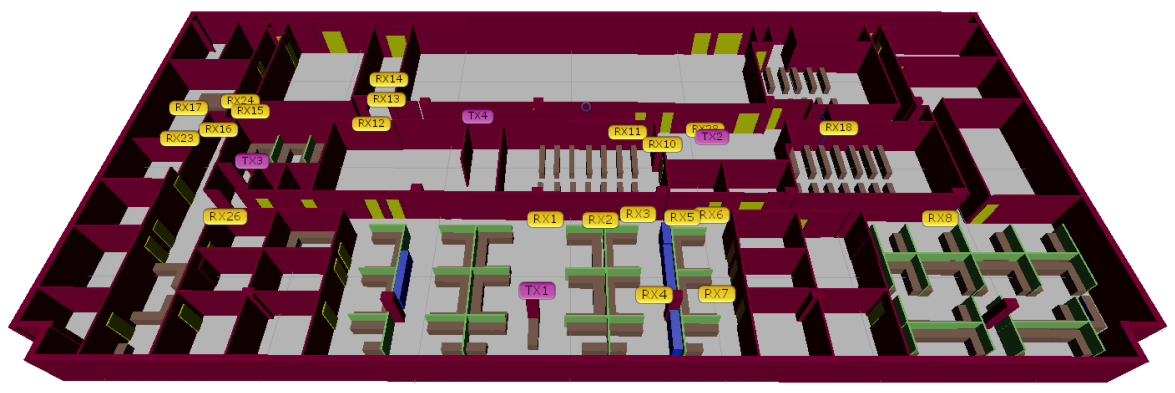

Figure 6.2: 3D scenario developed in Unity3D and Tx-Rx location. The materials characterized are concrete (red), drywall (green), metal (blue) and chipboard (brown)

of each material have been set for each and every one of them. Since metal, chipboard, drywall and glass have smooth surfaces, the Ward and Lambertian models have been used for them, while for concrete, which has a somewhat rough surface, the Ward and Oren Nayar models were selected. Furthermore, each one of the materials has been configured with a height map that emulates its roughness. Regarding the lighting rendering of Unity, it has been considered 32 direct samples, 512 indirect samples, 4 bounces, lightmap resolution is 40 texels per unit and the light map size is $1024 \times 1024$ pixels. Lightmap is set to high resolution. These settings are adequate to calculate direct and indirect illumination in scenarios with complex geometries according to [94].

\subsection{Evaluation Results}

The calibration process indicated in Section 6.3 has been applied to the indoor scenario, leading to the values of the lighting parameters shown in Table 6.1 and the polynomials for light intensity to path-loss translation for LoS and NLoS shown in equations (6.5) and (6.6), respectively, where $i$ is the normalized light intensity value.

$$
\begin{gathered}
P L_{L o S}[d B]=-10.44 i+128.8 \\
P L_{N L o S}[d B]=-0.45 i^{2}-3.23 i+109.9
\end{gathered}
$$

Figure 6.3 shows a scatter plot that for each one of the 24 measurement points indicates its measured path loss and its simulated normalized intensity. The measurements points in LoS are represented by blue circles while the 
Table 6.1: Calibration variable values for LIM

\begin{tabular}{|c|c|}
\hline Lighting variables & $\mathbf{2 8} \mathbf{~} \mathbf{H z}$ \\
\hline Light source intensity (LSI) & 2.75 \\
\hline Light bounce (LB) & 0.35 \\
\hline Shadow strength (SS) & 0.55 \\
\hline Shadow bias (SB) & 0.90 \\
\hline
\end{tabular}

measurement points in NLoS are represented by red squares. The figure also shows the polynomial functions obtained after LIM calibration for LoS and NLoS. The $R^{2}$ of the polynomial regressions are 0.93 and 0.83 for LoS and NLoS respectively, and the mean absolute error between the measured path loss and the path loss estimated by the LIM for all the measurement points is $2.20 \mathrm{~dB}$, which confirms the high accuracy of LIM in this case. The scatter plot also shows that points in LoS have high light intensity values and low large scale losses and are then concentrated on the right side of the graph. On the other hand, the points in NLoS are more dispersed over the entire light intensity range. Analyzing the estimation error in LoS and NLoS separately, we obtained that the mean absolute error in LoS is $0.94 \mathrm{~dB}$ and in NLoS is $2.83 \mathrm{~dB}$.

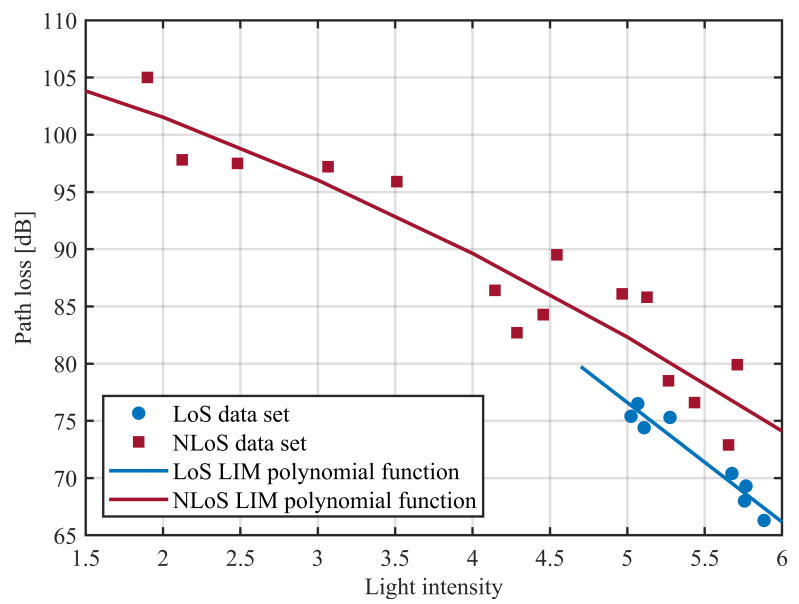

Figure 6.3: Path loss vs light intensity for the measurement points and the LIM polynomial functions 
Moreover, LIM has been compared with the NYU and 3GPP path loss models presented in Section 6.2. For the sake of a fair comparison, the values of the parameter $n$ for the NYU model have been recalculated compared to the values reported in [51] since the set of receiver points used in this work is a subset of the points used in [51]. As a result, the $n$ values used in this work are 1.15 for LoS and 2.42 for NLoS.

Figure 6.4 shows a scatter plot of path loss versus Tx-Rx distance in which a diamond marker is used to represent each path loss measurement and a square marker is used for each LIM estimation. Also the path loss versus distance curves for the NYU and 3GPP stochastic models are represented in Figure 6.4. It can be observed that the LIM estimation accuracy is independent of the distance and that its estimation is always close to the measured data. Concerning the stochastic models, the NYU is closer to the measured points.

For the sake of comparison between LIM and the NYU model, three points referred to as $\mathrm{A}, \mathrm{B}$, and $\mathrm{C}$ in the scatter plot of Figure 6.4 have been selected because of the high error of the NYU model to predict their path loss. The points A, B, and C correspond to the TX-RX combinations TX4-RX13, TX4RX14 and TX2-RX12, respectively (see Figure 6.2). The distance between TX4 and A is $8.2 \mathrm{~m}$, while it is $10.8 \mathrm{~m}$ between TX4 and B. It can be observed that, despite being relatively close to the transmitter, the NYU model presents a high error of $12.56 \mathrm{~dB}$ and $15.57 \mathrm{~dB}$ for $\mathrm{A}$ and $\mathrm{B}$, respectively, while LIM has an error of $1.55 \mathrm{~dB}$ and $2.96 \mathrm{~dB}$. The peculiarity of both points is that they are reached by a very small amount of rays from the transmitter due to its position after a corner where the rays have to traverse a narrow door space. Point $\mathrm{C}$ is located in a corridor, at a distance of $28.5 \mathrm{~m}$ from TX2. Despite the distance, the receiver is reached by a relatively high amount of reflected rays from the transmitter due to the reduced obstruction of the corner producing the NLoS condition and the location of RX12 in a narrow corridor which guides the reflections to the receiver. For this case, the NYU model error is $12.41 \mathrm{~dB}$, while the LIM error is only $3.74 \mathrm{~dB}$. All in all, the NYU model fails in special situations in which an abnormal amount of rays reach the measurement point, compared to other points at a similar distance and type of sight, due to an specific geometry of the obstacles and scatterers between the transmitter and the receiver.

Table 6.2 presents some statistics related to the error of the different models. The mean absolute error values obtained in the path loss estimation are $2.20 \mathrm{~dB}$, $3.94 \mathrm{~dB}$ and $8.47 \mathrm{~dB}$ for LIM, NYU and 3GPP, respectively. There are very slight differences between LIM and NYU model in LoS environments (0.93 dB and $1.69 \mathrm{~dB}$, respectively) that are reasonably low. By contrast, the 3GPP model has a high absolute error of $5.47 \mathrm{~dB}$. For NLoS environments, LIM is significantly better than 3GPP and NYU models. When it comes to the 
maximum absolute error, LIM outperform significantly the other two models. Its maximum absolute error is only $4.92 \mathrm{~dB}$, compared to almost $20 \mathrm{~dB}$ for the other two models.

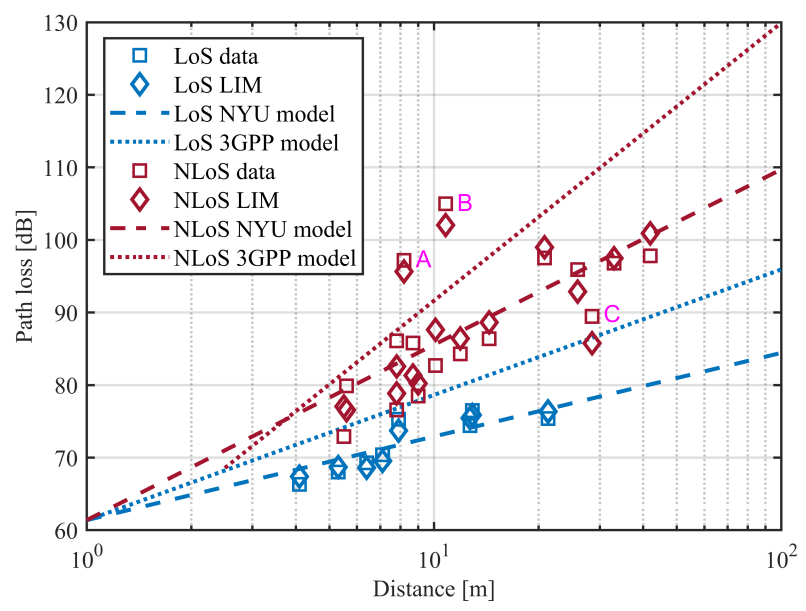

Figure 6.4: Path loss versus Tx-Rx distance for stochastic models, NYU measurements and LIM estimation

Table 6.2: Absolute error comparison for LIM and stochastic models

\begin{tabular}{|c|c|c|c|c|}
\hline \multirow{2}{*}{ Model } & \multicolumn{4}{|c|}{ Absolute error [dB] } \\
& Global mean & LoS mean & NLoS mean & Max. \\
\hline LIM & 2.20 & 0.93 & 2.83 & 4.92 \\
\hline NYU & 3.94 & 1.69 & 5.07 & 15.57 \\
\hline 3GPP & 8.47 & 5.47 & 9.97 & 19.55 \\
\hline
\end{tabular}

Figure 6.5 shows the Probability Distribution Function (PDF) of the absolute error. It is noted that the LIM and NYU models estimate propagation losses better, which is expected since they are calibrated in the same scenario where the evaluation is conducted, while the $3 \mathrm{GPP}$ model shows a high error probability. LIM and NYU perform similarly but NYU shows a non-negligible probability of having high errors since it does not accurately captures some special situations of the realistic propagation. 


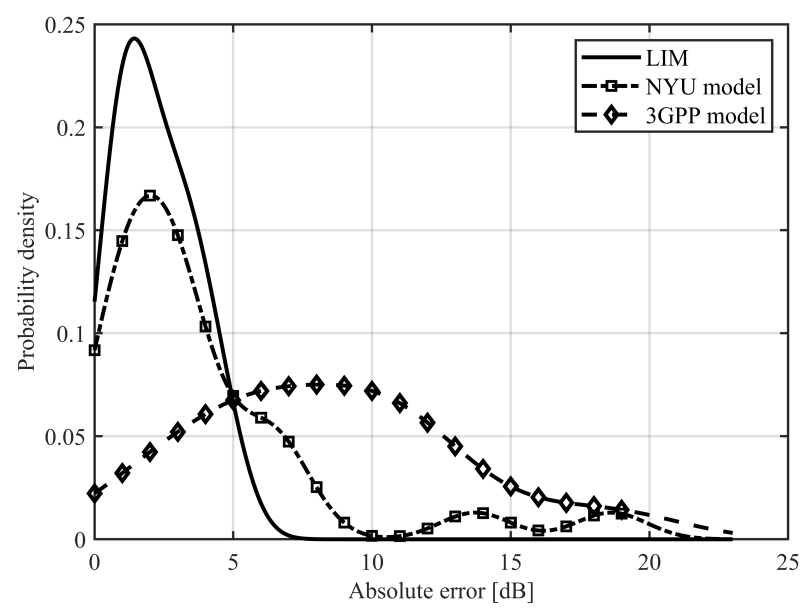

Figure 6.5: Probability distribution of the absolute error in the path loss estimation

\subsection{Conclusions}

In this chapter, we have compared LIM with two stochastic path loss models for indoor environments. Concretely, a 3GPP based path loss model, and a path loss model obtained by the NYU. Simulation results show that LIM outperforms the other two models. Specifically for LoS, NYU model and LIM get very accurate path loss values, while the $3 \mathrm{GPP}$ model is overestimating the losses. Moreover, in NLoS the good results of LIM are accentuated both in terms of mean error and above all in the worst case, for the maximum error.

From these outcomes, we can conclude that LIM is a valid and simple approach to obtain accurate estimates in indoor scenarios known with a high level of detail due to its capacity to correctly capture the peculiarities of the environment. Using generic stochastic models such as the 3GPP model is not valid for the channel modeling in a real indoor scenario. On the other hand, an stochastic model calibrated in a real scenario, is able to provide better results, and would be encouraged in case of using a stochastic model but, it would fail in some specific situations due to peculiarities of the scenario geometry.

In this work, measurement points where transmission and diffraction are the main propagation mechanisms have been excluded from the assessment because these mechanisms are not considered by the current LIM implementation. Future work will include the consideration of transmission and diffraction mechanisms by LIM. Transmission will be included modifying the transparency of 
CHAPTER 6. LIGHT INTENSITY MODEL EVALUATION IN INDOOR SCENARIOS AT $28 \mathrm{GHZ}$

the materials, while diffraction is planned to be emulated by adding secondary sources of light. 


\section{Chapter 7}

\section{A Multi-Purpose Simulation and Visualization Platform for 5G based on Unity}

This chapter introduces the simulation and visualization platform developed in this Thesis based on the Unity game engine. It will present several use cases implemented in the platform, highlighting the benefits of Unity as a visualizer and as a general simulation platform of $5 \mathrm{G}$ systems.

To that end, this chapter has been divided into the following sections:

- Section 7.1 introduces the simulation and visualization platform presenting its main characteristics.

- Section 7.2 describes the main building blocks of the simulation and visualization platform developed.

- Section 7.3 describes the use cases implemented in the platform and its main features.

- Section 7.4 draws the main conclusions of the chapter. 


\subsection{Introduction}

It is common to find wireless communications simulation platforms that are limited in their visualization concepts. Most of the simulation tools available for mobile networks have an abstract visualization graphical interface, usually in $2 \mathrm{D}$ or with limitations in their $3 \mathrm{D}$ graphical environment and without real time interaction with the user [119]. These traditional visualization tools have important limitations, such as the lack of interactivity or the absence of immersion of the user. In that sense, one thing that should be taken into account for the development of future $5 \mathrm{G}$ simulation tools, where the interaction between network components, users and devices is multiplied, is to develop visualization tools and immersive simulations. That will allow to achieve a better understanding of the network. In that sense, it is necessary to identify the reasons why improving the visualization tools of $5 \mathrm{G}$ networks is important:

- It allows a better understanding of the use case and helps to make decisions of those responsible for technical solutions.

- It allows a better understanding of the interaction between the components of the network compared to abstract simulators.

- It simplifies the definition, implementation and parameterization of complex 3D use cases and scenarios.

- It simplifies the visualization of the results and allows to experience the use case in first person, generating a better perspective of the problem.

- It allows to evaluate critical use cases that are costly and complicated to simulate as emergency scenarios, security, industry or vehicular communications.

- It allows a dynamic interaction with the user in which changes can be made in real time.

One of the most important advantages of using Unity's game engine is the great potential of its graphic engines to achieve super realistic scenes and emulate real environments and conditions. In that sense, this chapter aims to highlight and show the capabilities of the Fifth Generation (5G) system simulator developed in this Thesis using Unity's game engine. The objective of the simulation and visualization platform is to implement and emulate the main use cases of $5 \mathrm{G}$ within super realistic scenarios for a use from the scientific point of view, to the marketing or academic point of view. The development of this platform, shares the objectives of Mobile and wireless communications 
Enablers for Twenty-twenty Information Society II (METIS-II) project; in fact, part of this work has collaborated with the visualization platform of METIS-II project, available in [99, 120]. Nevertheless, the METIS-II platform was limited to a single realistic scenario and provided good user interaction and immersion in the game, but was limited to the graphical representation of simulation data from an external source conveniently formatted to the platform. This Thesis expands the METIS-II visualization concepts for $5 \mathrm{G}$ networks, in which the following points are highlighted:

1. The scenarios used for the simulations are super realistic, where in addition to considering the exact geometry of the scenario, dynamic behaviors can be added, such as pedestrian or vehicular flow.

2. It allows to evaluate critical use cases that are costly and complicated to perform as emergency scenarios, security, industry or vehicular communications. Network Metrics calculations are obtained in real time.

3. Real-time interaction with network components.

4. A characterization of the scenario and radio propagation conditions very close to reality.

5. Immersive user experience. Implementation of first-person visualization and Virtual Reality compatibility.

6. Regarding the estimation of radio propagation, ray-tracing and Light Intensity Model (LIM) algorithms have been optimized for a correct evaluation of the radio channel.

7. The application is based on Serious Game Engineering, therefore, it is oriented to simulations with professional level.

\subsection{Platform Description}

Figure 7.1 shows the general layout of the simulator. The Block I is where the external inputs of the simulator are processed, these can be from peripherals to external programs such as Matlab, Python or others. Blocks II, III and IV are where the information is processed, this process can be done in real time or with pre-calculated simulations. The visualization process and the generation of the output files correspond to blocks V and VI respectively. Each of the components is detailed below: 


\section{CHAPTER 7. A MULTI-PURPOSE SIMULATION AND VISUALIZATION PLATFORM FOR 5G BASED ON UNITY}

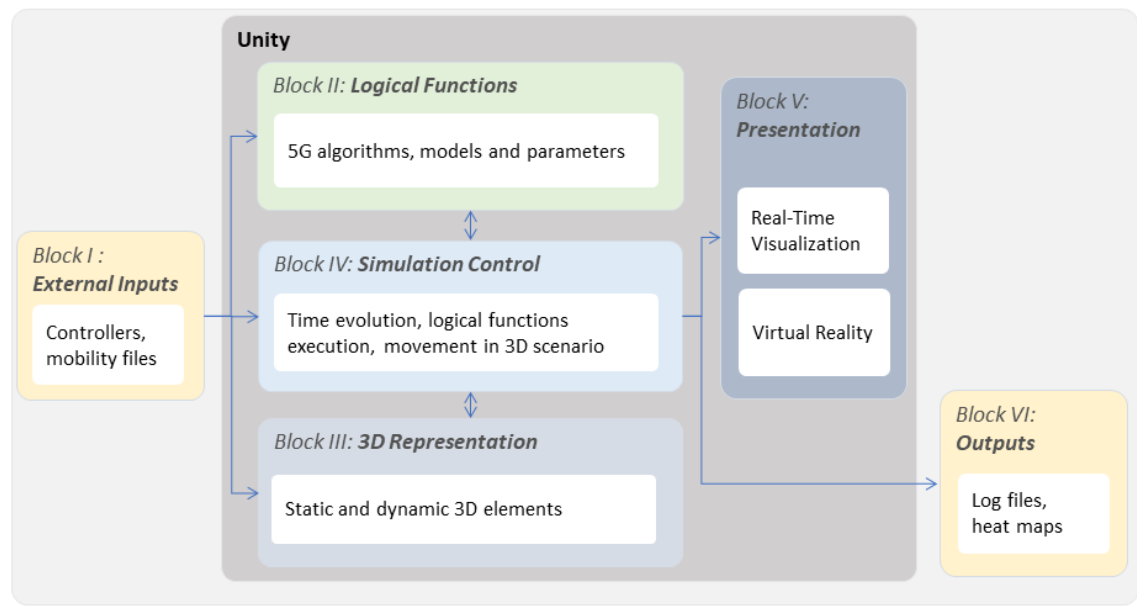

Figure 7.1: Simulator layout

\subsubsection{Block I: External Inputs}

Unity enables the deployment of multiple input devices ranging from conventional peripherals such as joypads, keyboards, and controllers to virtual reality, augmented reality, and motion-sensing system inputs. It also considers external inputs to platforms, programs or files that interact offline or in real-time with Unity. For example, the Mobile and wireless communications Enablers for Twenty-twenty Information Society (METIS) simulator uses external files with the trace of movement for pedestrians and vehicles $[69,121]$. In the vehicular communications field, interfaces have been developed between micro simulators such as Simulation of Urban Mobility (SUMO) or Multi Agent Transport Simulation (MatSIM) [122] that allow the generation of realistic traffic environments and that, together with Unity, allows the immersion of traffic within a realistic environment. In fact, in this field, our simulator (Section 7.3.2) goes further and implements first-person immersion for a more realistic simulation and implementation of more use cases. In the field of artificial intelligence, Unity developed Maching-Learning libraries, which have an interface with Python. In fact, several applications are being developed in this area. For example, simulation projects and training of an autonomous car, with high sensory complexity in urban environments, with the capacity to interact with multiple agents [123]. There are other industrial applications, which seek to implement the concepts of Maching Learning in industrial processes, for example, an IBM extension is available, called IBM Watson for Unity, that allows integrating services such as 
visual recognition, conversion from voice to text and classification of languages [124]. This capability of the Unity platform opens a range of possibilities for the development of applications not only in the video game sector, but also for applications developed under the concept of Serious Game Engineering.

\subsubsection{Block II: Logical Functions}

This block contains all the algorithms and mathematical models for the $5 \mathrm{G}$ use cases. These algorithms are found in libraries and plugins, can be used independently or interact with each other, depending on the requirements. In the first version of the simulator, for the radio channel we have the following elements:

- Pattern of synthetic and real antennas, configuration of tilt and azimuth, power of TX and RX.

- Deterministic propagation models: Ray-Tracing, METIS Map-Based Model, LIM.

- Stochastic propagation models: Third Generation Partnership Project (3GPP), International Mobile Telecommunications 2020 (IMT-2020), New York Univeristy (NYU).

- Small scale models: Ray-tracing, IMT-2020, NYU.

- Electrical configuration of materials for ray-tracing, and textures and material light properties for LIM.

\subsubsection{Block III: 3D Representation}

This is one of the most important blocks. The scenario is created here and all the dynamic elements are set up. The scenario in Unity, as explained in the Chapter 3, could contain 3D models that are available for free in the galleries of Google Earth, Sketch Up or others and speed up the development process. The quality and precision of the 3D elements of the scenario are categorized according to their importance within each use case. As shown in the diagram, the active elements that are directly linked to the use case have priority in the level of detail, texturing and rendering. The background elements are represented generally by regular forms to speed up the simulation process. Figure 7.2 shows an example of a complex scenario developed in Unity for the evaluation of vehicular communications.

Dynamic elements have a special treatment. They are elements with mobility that influence the calculation in the cases of use, and their configuration 
CHAPTER 7. A MULTI-PURPOSE SIMULATION AND VISUALIZATION PLATFORM FOR 5G BASED ON UNITY

has to be done under real models. Figure 7.3 shows the detail of a complex scenario, where traffic management has been carried out and several dynamic elements have been configured.

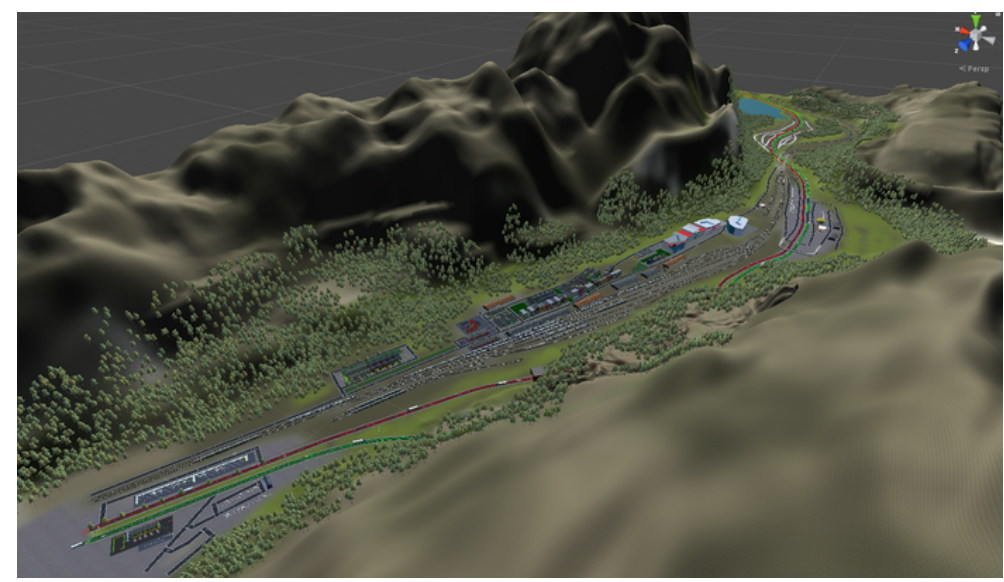

Figure 7.2: Real 3D scenario developed in Unity

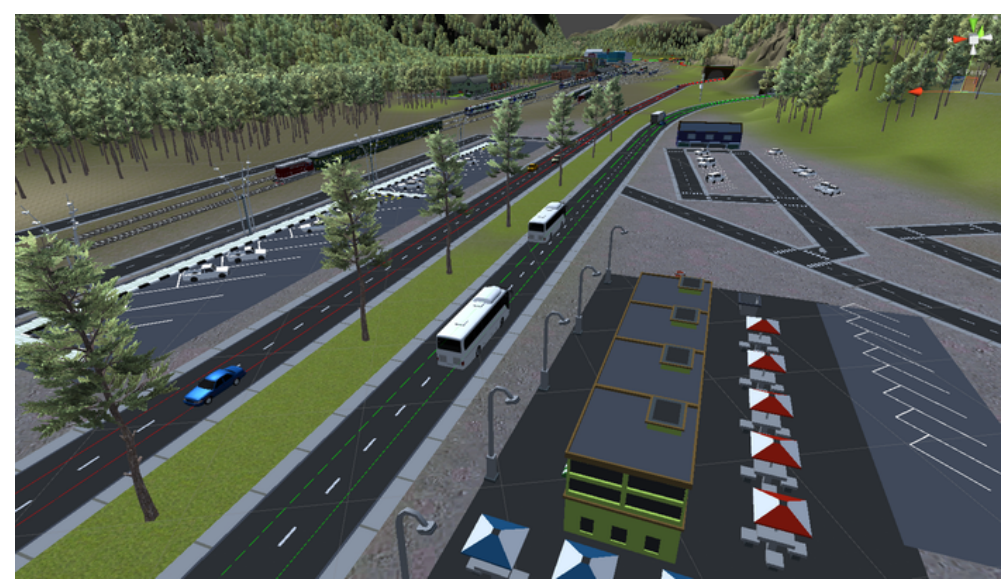

Figure 7.3: Dynamic components in a real scenario developed in Unity 
The use cases currently implemented in the platform are:

- Planning of coverage of mobile networks in the Millimeter Wave (mmW) bands.

- Ray-tracing for $\mathrm{mmW}$ in super realistic scenarios.

- Autonomous Driving.

- Vehicular communications.

- Unmanned Aerial Vehicle (UAV) mobile base station with artificial intelligence algorithms for emergency situations.

\subsubsection{Block IV: Simulation Control}

The simulation process is in charge of carrying out the temporal evolution of the use case and data collection. In this process, all the functions of the logic layer are executed and updated in each frame. This simulation process is the one that takes most of the time. In fact, depending on the use case, it can take hundreds of hours of processing. For example, for applications that need to implement training algorithms and artificial intelligence, this process can be extended. On the other hand, for real-time processes such as the calculation of propagation losses, this process can be performed in real time. For complex cases, it is possible to speed up this process by deactivating the graphic component of the platform and working in console mode. In fact, in this stage it is foreseen to use only the essential components of the use case.

\subsubsection{Block V: Presentation}

This block corresponds to the visualization interface between the user and the simulation platform. In general, the presentation layer should be friendly, intuitive and easy to use. On the other hand, this is where the dynamic components become relevant, because the user must be able to interact with them. The presentation layer must provide an enhanced immersion experience. The visualization is developed according to the use case. Figure 7.4 shows an application developed for the use of UAVs as base stations for emergency situations. Two parts can be identified in this visualization. The first one corresponds to the 3D scenario where the fixed and dynamic components interact. The second part corresponds to displaying the simulation results. 


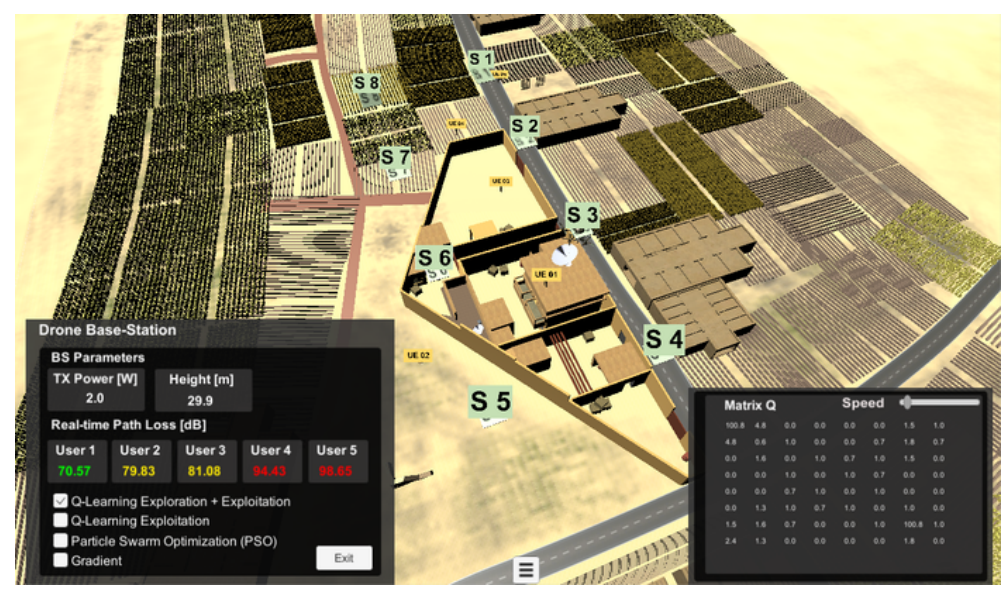

Figure 7.4: Example of a presentation layer in Unity

\subsubsection{Block VI: Outputs}

The platform's output files allow a deeper analysis of the usage case. The files can include maps of propagation losses that represent the losses in $\mathrm{dB}$ from the position of the fixed station to a set of points distributed in a specific $x-y$ plane in a $\mathrm{z}$ coordinate, maps of mobility of users and devices, for radio propagation can include detail of the multipath as the Angle of Departure (AoD), Angle of Arrival (AoA). All in all, the output files are dependent on the implemented use case.

\subsection{Use Cases}

\subsubsection{Planning of coverage of mobile networks in the mmW bands based on LIM}

This section shows a tool from the simulation and visualization platform to calculate coverage maps of $5 \mathrm{G}$ antennas in super realistic environments. The tool is valid for frequency bands above $6 \mathrm{GHz}$ and for Urban Macro-cell (UMa) and Urban Micro-cell (UMi) scenarios. After choosing an antenna location or site in a specific region, the coverage map allows network planning engineers to visually detect areas where the coverage is poor or, more critically, nonexistent. Coverage maps are traditional heat maps representing the amount of received power, the propagation loss, the expected service quality, or any other useful metric. These maps help engineers to plan but also to optimize the 
radio network coverage. Any change in the region orography may completely disrupt the service in an area. Professional planning tools have been in the market for many years and they create precise coverage maps using complex ray-tracing algorithms. However, in general, software licenses of such powerful tools are expensive. This use case of the platform aims at offering a parameter tuning similar to professional software. The higher calculation speed of the platform largely compensates the small difference in prediction accuracy with professional software, which is suitable to have a fast idea on where coverage problems may occur. Figure 7.5 shows an initial view of the simulation and visualization platform with the game menu and the scenario in the background. As already stated, the objective of this demo of the simulation and visualization platform was to instruct on site planning for $5 \mathrm{G}$ millimeter waves networks. The game user could display the coverage areas of the different transmitters used in the measurement campaign area. By clicking on the "Custom Location" in the menu, the User Equipment (UE) is able to select an arbitrary location on the scenario for a new transmitter. Then, the simulation and visualization platform places a 3D model of the antenna with the "TX-Custom" legend below.

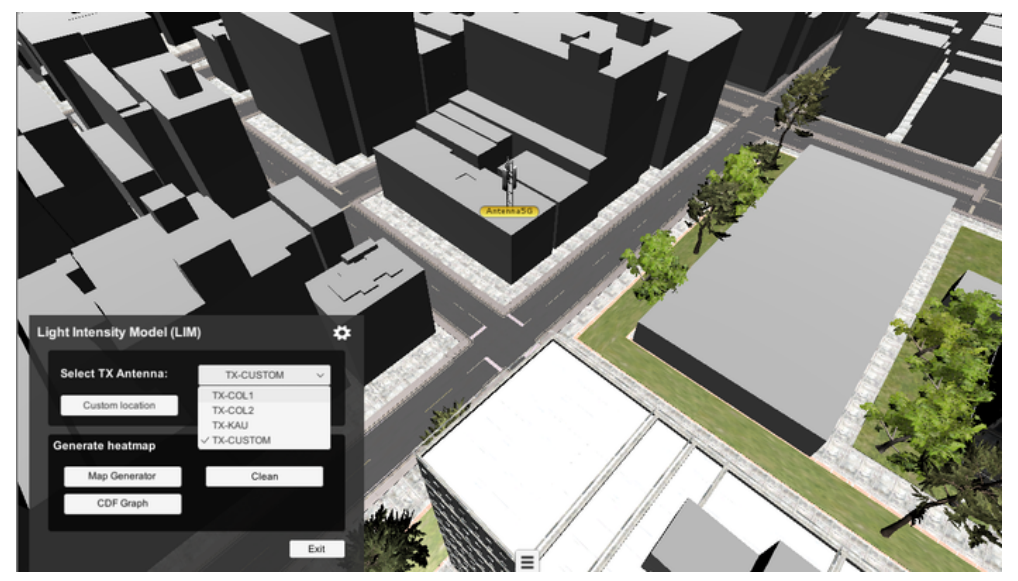

Figure 7.5: 5G planning tool - LIM

The user can visualize the coverage map of the newly deployed antenna by selecting the "Map Generator" button. This is a two-step process where 1) the simulation and visualization platform first calculates the coverage map in real time with LIM of a single antenna; and 2) represents the map on the scenario. Internally, the coverage map is a grid of points with propagation losses in the virtual scenario. These points are distributed over an $\mathrm{x}-\mathrm{y}$ plane at a specific $\mathrm{z}$ coordinate or height of $1.5 \mathrm{~m}$, which is the typical value used for users on the 
ground in station-to-user communications. The loss map comprises as many rows as loss samples in y-axis, and as many columns as loss samples in x-axis. The set of points whose loss values are represented in the loss map are assumed to be the central points of the rectangles obtained as a result of the division of the scenario under study in a grid with a specific x-spacing and y-spacing.

After calculating the coverage map, the simulation and visualization platform fills the grid of points with squares of a certain color according to the chosen color map. The grid granularity influences the speed of data visualization as the larger the number of squares to represent the more time it takes to the visualization tool to display all of them. The use of wider squares reduces visualization timing but results in rendering a pixelated coverage map. The effect of using wider measurement spacing is shown in Figure 7.6, where granularity was set to $5 \mathrm{~m}$ in both axes. Other visual information may also appear in the simulation and visualization platform like, for example, tags identifying the location of the transmitting antennas and the measurement points needed for calibrating LIM. This information can be stored in log files in order to perform a posteriori data processing; similar to the drive tests mobile network operators performs. Drive tests are very useful for operators since they allow detecting issues in the radio access network, which would not possible to detect otherwise. This is the case of coverage holes due to changes in the propagation conditions. Typically, they are corrected by tuning the transmission parameters such as the transmit power.

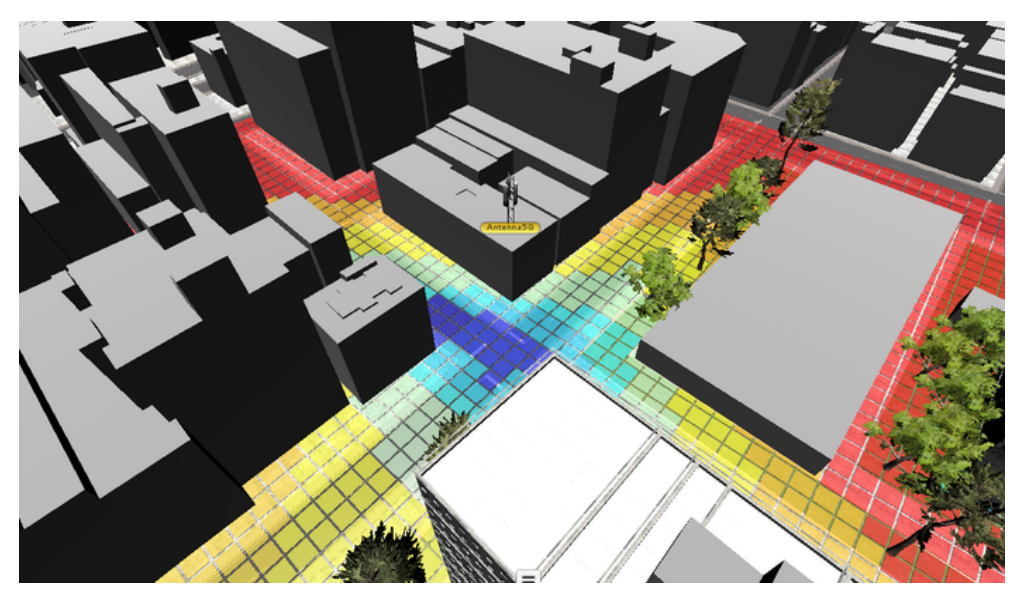

Figure 7.6: 5G planning tool - LIM 


\section{Virtual Drive Test Experience}

The simulation and visualization platform also offers a real-time drive test option to provide an enhanced immersion experience. This feature gives the possibility to be an active part of the simulated scenario and control a pedestrian with a third-person view. Figure 7.7 illustrates the simple case where the simulation and visualization platform user selects the serving antenna and displays the evolution of the propagation conditions as the user (a capsule) moves along the scenario. Propagation data is calculated with the LIM module on every frame based on the current player location, and the path loss value is dynamically displayed on the User Interface (UI).

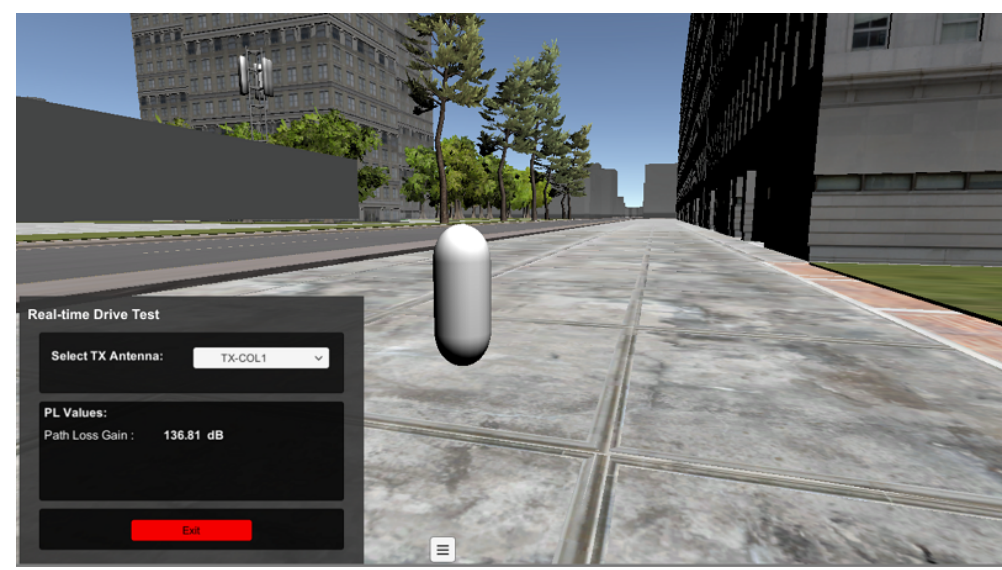

Figure 7.7: Real-time drive test

The simulation and visualization platform can also modify the data obtained with LIM to show something more sophisticated than signal levels. In Figure 7.8, the player, which is a pedestrian model, walks around the virtual scenario while playing an Ultra High Definition (UHD) video a smartphone. In the example, the UI displays in a graph the real-time evolution of the download speed. This way, the user of the simulation and visualization platform may have a perception of the quality of experience as it walks and suffers fluctuations on the channel conditions.

\subsubsection{Vehicular Communications Simulation Tool}

According to Cisco's report [125], mobile video traffic will experience the highest growth by 2021. Mobile video will represent $78 \%$ of global mobile traffic. And, the consumption of live mobile video traffic will represent $5 \%$. In this 


\section{CHAPTER 7. A MULTI-PURPOSE SIMULATION AND VISUALIZATION PLATFORM FOR 5G BASED ON UNITY}

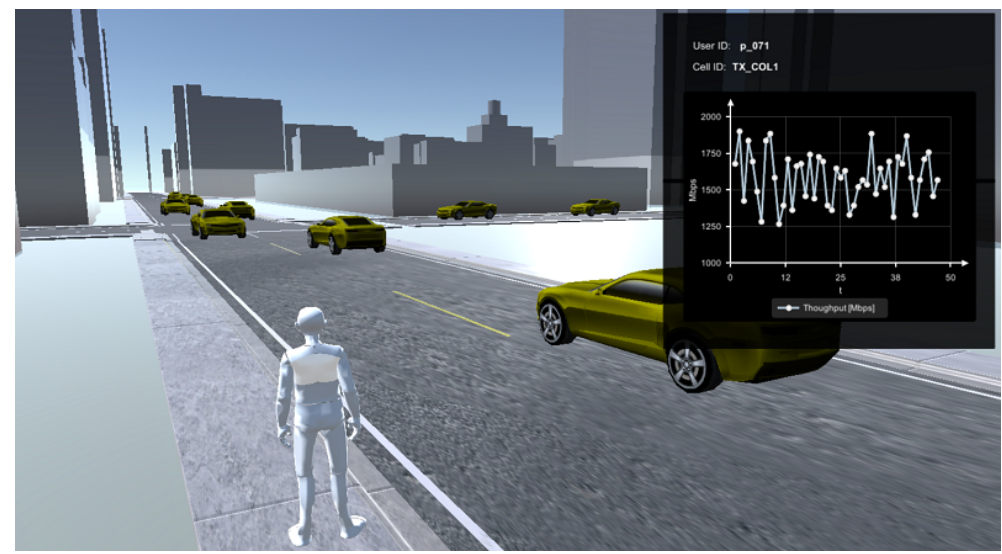

Figure 7.8: Example of a pedestrian user with a graph showing the evolution of the experienced data throughput in real time

context, it is correct to foresee that also the consumption of mobile video will experience a growth in the users of autonomous cars. In fact, from now on it is a necessity for passengers to use mobile networks where video data consumption is frequent.

It is therefore important to evaluate this use case and explore the different architectures and configurations of the $5 \mathrm{G}$ networks in order to achieve the Quality of Service (QoS) values that guarantee high quality streaming transmission without interruptions. The implemented simulation has several evaluation fronts, listed below:

1. Simulation and evaluation of the capacity of the radio channel in a super realistic scenario.

2. Analysis of propagation losses in a highway tunnel using ray-tracing and LIM models.

3. Hand-over analysis between femto cells of the same operator and QoS evaluation.

4. As the simulation scenario is set between a border zone of countries, it is expected to estimate the inter-operator hand-over time and evaluate the best solutions to mitigate the interruptions in this scenario.

Figure 7.9 shows the simulator. In this use case, the values of the propagation losses are updated every frame for a correct calculation of the radio 
capacity. Furthermore, it is a starting point for a better understanding of the problem raised. The video degrades between the snapshots 1 and 2, and then, in 3 reaches its most critical point inside the tunnel, where the connection is lost. Then, in 4, it recovers adequate QoS values at the end of the tunnel. It should be noted that there are two simulation modes. One simulates autonomous driving and the other where the user can drive freely, as if it was a video game.

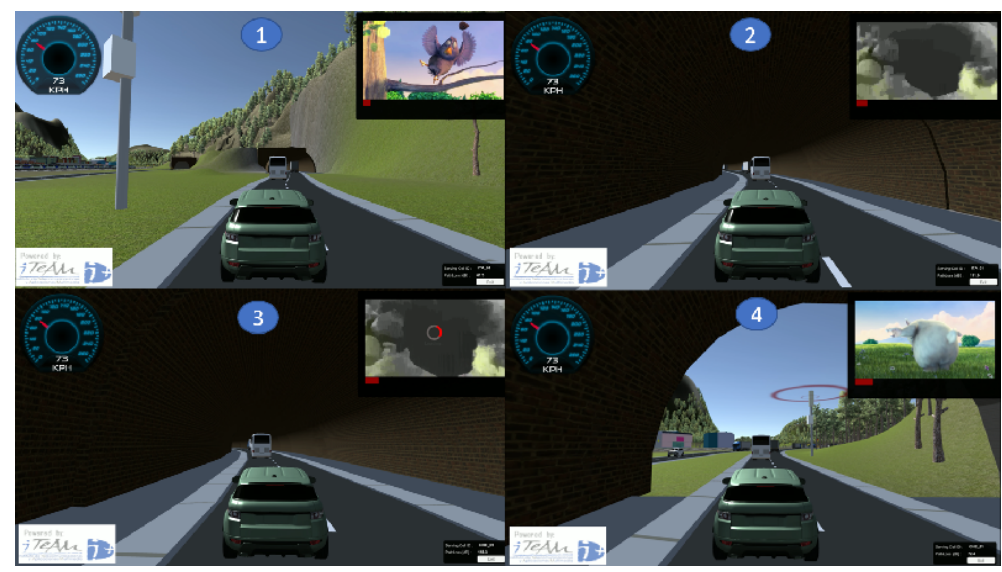

Figure 7.9: Use Case: Real-time video streaming behavior

\subsubsection{Ray-Tracing Simulator for $\mathrm{mmW}$}

The ray-tracing simulator developed at Unity is a high-precision channel modeling tool. This simulator shows a visualization layer that differentiates it from other conventional ray-tracing simulators.

The default ray-tracing simulator has a synthesized antenna pattern implemented for both transmitter and receiver. It is possible to build different antenna structures for example monopoles, dipoles or patches. Also, the geometrical construction of arrays of antennas is feasible. The materials available for the simulations are described in Appendix A. In addition, a variable corresponding to the thickness of the materials has been added for the calculation of reflection coefficients in indoor scenarios. The location of the TX and RX can be positioned from the visualization platform. Figure 7.10 shows the simplified user interface for estimating propagation losses in real time using ray-tracing algorithms. 


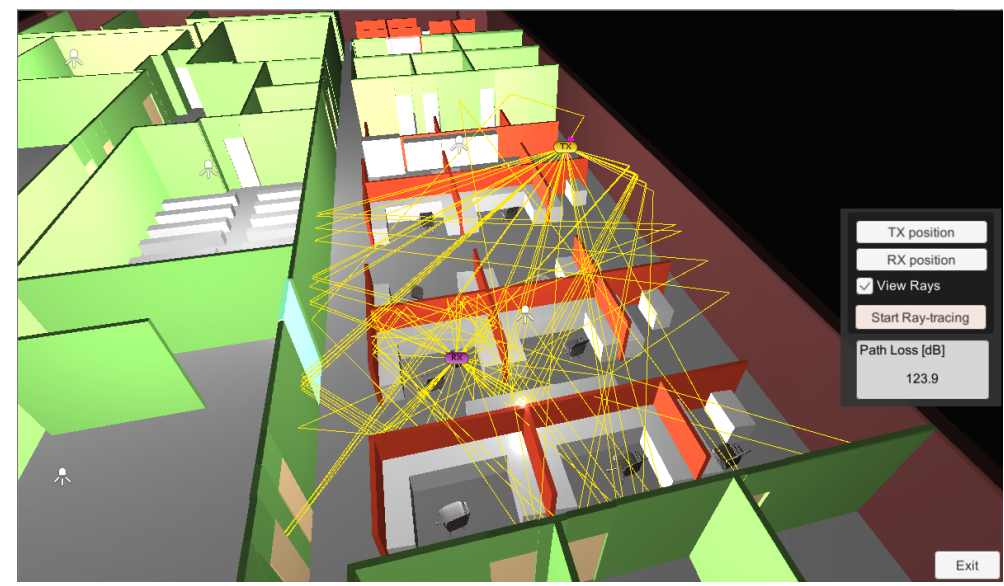

Figure 7.10: Ray-tracing based on Unity 3d

The ray-tracing simulator is highly accurate for the estimation of the radio channel, as seen in Chapters 4 and 5 . In this section the visualization capability of ray-tracing implemented in a gaming platform is highlighted. For example, Figure 7.11 shows the simulator evaluating the components of diffuse scattering. The same can be done with specular or direct components. The purpose of the simulator is to improve the levels of understanding of the radio channel.

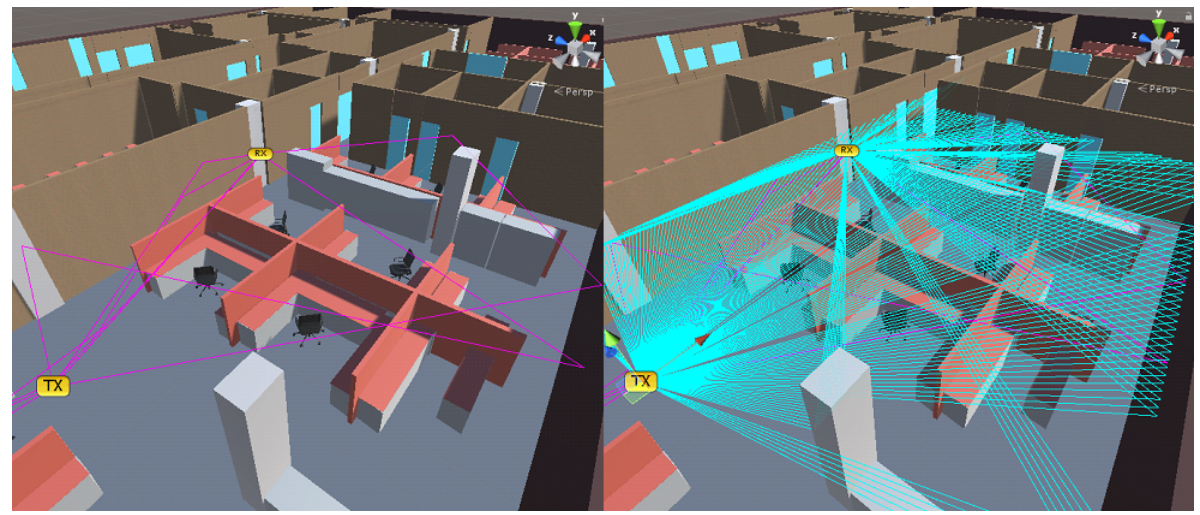

Figure 7.11: Detailed modeling of diffuse scattering in indoor channels for 5G wireless networks 


\subsubsection{UAV Mobile Base Station with Artificial Intelli- gence Algorithms for Emergency Situations}

This section shows a tool from the simulation and visualization platform to optimize the location of an UAV mobile base station with different user mobility patterns and using artificial intelligence algorithms. This tool has been used to conduct the assessment published in [126], in which the author of this Thesis was responsible of the creation of the $3 \mathrm{D}$ scenario, the channel modeling, the implementation of the user mobility patterns, and the control of the UAV mobility.

Currently, aerial base stations are being considered in the deployment of wireless networks with many purposes. For example, in emergency responses and public safety situations such as earthquakes, hurricanes, fires or in military operations where terrestrial communications networks are damaged or not fully operational, a fast deployment of communication systems is crucial. Seizing the versatility of Unmanned Aerial Vehicles (UAVs), instant networks based on them are often performed for these cases since the UAV networks can overfly the area very quickly avoiding physical obstacles [127]. In that sense, the purpose of this tool is to optimize the location of an UAV mobile base station with different user mobility patterns. To this end, an urban scenario has been deployed following the urban high-rise environment and the user mobility patters are emulated as shown in Figure 7.12.

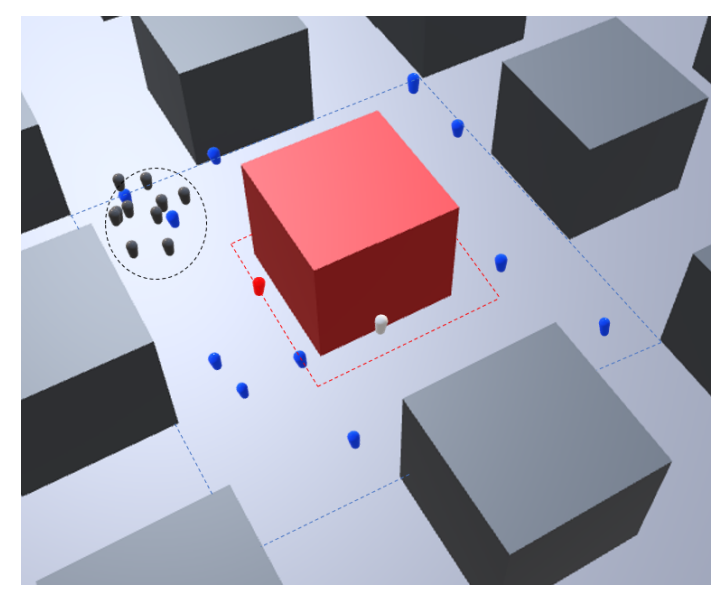

Figure 7.12: 3D scenario developed in Unity and users mobility patterns

Briefly, the set of users in blue perform a random movement over the whole area being uniformly distributed. User in red is a single user circling around the 
central building. And the black users at the beginning are randomly distributed in the scenario and as the simulation progresses all the users move to the same zone delimited by a dotted line in gray where they stand still. The objective in this scenario is to minimize the path loss experienced by the user with the highest path loss, i.e. to maximize the signal power of the user with the worst channel. Optimization consists of finding the best location for the UAV through the training of three machine learning algorithms: a Q-learning technique, a Gradient-based solution and a Greedy-search solution.

Simulation results show that the performance of the three methods is similar when a high number of users move randomly and uniformly around the scenario under test. Nevertheless, in some situations when the number of users is reduced or when the users move together in a similar direction, both Gradient and Greedy algorithms present a significantly better performance than the Q-learning method. This tool is a very useful example to demonstrate that this simulation and visualization platform is versatile and has great potential for the simulation of highly complex use cases.

\subsection{Conclusions}

In this chapter a $5 \mathrm{G}$ simulation and visualization platform developed in this Thesis has been presented. It has been implemented in the game engine of Unity under the concept of "Serious Game Engineering". Several use cases are currently included within this platform for the $5 \mathrm{G}$, ranging from radio planning to use cases with vehicular communications. An important part of the simulation part of the platform is its LIM implementation which allows a very fast evaluation of the path losses and hence a real time simulation. The implementation of the different use cases has demonstrated the great potential that Unity has to be used as a general platform for $5 \mathrm{G}$ mobile communications systems. The benefits of Unity as a visualizer and as a general simulation platform lie in its flexibility, in fact, the use cases that can be implemented have no restrictions. In the framework of serious games, this section has introduced a visualization tool aiming at explaining telecommunication engineering concepts for the next generation wireless communication standard. This simulation and visualization platform has the flexibility and the resources to represent relevant data for a number of applications from engineering, analysis and design points of view. 


\section{Chapter 8}

\section{Conclusions and Future Work}

\subsection{Concluding Remarks}

This doctoral Thesis had two main objectives. The first one was to use the game engines to evaluate the propagation losses in the millimetre-wave bands based on the propagation of light in the illumination of the scene. The second one refers to the use of the game engine as a simulation platform for the different use cases that the Fifth Generation (5G) proposes.

Concerning the first objective, a channel model known as Light Intensity Model (LIM) has been designed for the estimation of large-scale propagation losses for the bands above $6 \mathrm{GHz}$. Using this model, based on the lighting processes performed by the game engines, the signal transmitters are replaced by light sources and the light intensity received at a point is translated into signal strength in mmW through a simple polynomial function. The LIM model has been calibrated, i.e. its polynomial function has been fitted to real measurements, in the 28 and $73 \mathrm{GHz}$ bands, and their predictions have been compared with ray-tracing, map-based and stochastic models valid for the bands above $6 \mathrm{GHz}$. The obtained results have shown, on the one hand, that the LIM is more accurate than semi-deterministic and stochastic models. This accuracy increases at higher frequencies, which corroborates the intuition that the LIM could be more accurate at wavelengths closer to that of light. On the other hand, it has been proved that the computational cost of the LIM is considerably lower than that of ray-tracing, which enables the use of the LIM in real time and in highly complex scenarios. Specifically, the computational cost 
of the LIM compared to ray-tracing is 130 times lower. This great improvement is due to the simplification that the light model entails with respect to a massive ray launch, as well as the efficient use made by the light model of the computer's graphic computing systems.

In the first set of studies about the LIM and in its comparison with other models, diffuse scattering effects were not explicitly considered. Nevertheless, a number of works indicate clearly the importance of such phenomenon on path loss estimation. Therefore, the LIM has been extended to explicitly consider diffuse scattering. The game engines allow characterizing in a different way the interaction of the materials with the light configuring the normal map of their surfaces and their scattering and reflection functions. Based on this capability of the game engines, the idea is to have a diffuse scattering of the light in the game engine similar to the diffuse scattering of the radio waves. To evaluate that approach, the ray-tracing implementation used in this Thesis has been extended to take into account diffuse scattering. Both, ray-tracing and LIM have been then calibrated using as reference laboratory measurements made in a controlled scenario in the $28 \mathrm{GHz}$ band. The extension of the LIM model using materials with this optimal characterization reduces by more than $50 \%$ its estimation error with respect to the application of the LIM with default materials. In fact, the Root Mean Square Error (RMSE) compared to raytracing is only about $0.5 \mathrm{~dB}$ higher. In exchange of this higher accuracy, the computational cost of the LIM implementation increases, but it remains 26 times lower than the cost of the ray-tracing implementation.

Concerning the second objective, a simulation platform for the $5 \mathrm{G}$ has been designed and several use cases of this platform have been presented. These uses cases include radio planning and real-time coverage study for millimeter waves using LIM, the study of propagation losses using the ray-tracing model, the simulation of complex scenes of vehicular communications, artificial intelligence, and others.

It is necessary to analyze each of the hypotheses formulated in this Thesis and indicate how this research work has answered each of them:

- Hypothesis 1: The propagation model based on light engines minimizes the error in estimating propagation losses in the millimeter bands compared to other models. In a first assessment, without considering diffuse scattering, the LIM has been compared with several stochastic and semideterministic models, and with a ray-tracing implementation in an urban scenario at $28 \mathrm{GHz}$ and $73 \mathrm{GHz}$. It has been observed that LIM is more accurate than stochastic and semi-deterministic models, but less accurate than ray-tracing. Nevertheless, the additional RMSE compared with raytracing for the LIM is just $1.49 \mathrm{~dB}$ at $28 \mathrm{GHz}$ and $0.46 \mathrm{~dB}$ at $73 \mathrm{GHz}$. In 
a second assessment, considering diffuse scattering, and focusing on the estimation of losses after reflection on a single material, still ray-tracing is more accurate than the LIM but it is only $0.5 \mathrm{~dB}$ better. In conclusion, the hypothesis is fully validated if the LIM is compared to low-complexity models, but it does not hold for the comparison with ray-tracing although the LIM accuracy is not significantly worse than ray-tracing.

- Hypothesis 2: The implementation of complex 3D scenarios in Unity3D improves the process of estimating propagation losses. In this Thesis the need to use super realistic 3D scenarios for a better evaluation of the radio channel was demostrated. In Chapter 2, stochastic and semideterministic models have been compared with propagation loss values obtained in different measurement campaigns for frequencies above and below $6 \mathrm{GHz}$. In both cases, the map-based model has shown a lower RMSE. Similarly, in Chapter 4, it has been shown that for both LIM and ray-tracing, it is necessary to have scenarios that contain detailed geometry and information on the materials present in the scenario. Finally, in Chapter 6, the need for complex scenarios has been demonstrated for a better evaluation of the use cases proposed by $5 \mathrm{G}$.

- Hypothesis 3: LIM is computationally efficient compared to complex raytracing models. In this Thesis, its has been confirmed that the computational complexity of LIM becomes its best advantage. Precisely, in the results obtained in the comparison of LIM with ray-tracing, it is demonstrated that the amount of flops needed by ray-tracing is almost 130 times higher than those needed by the LIM, for the prediction of losses in the urban scenario, which translates into less processing time and less use of Central Processing Unit (CPU) resources. Even, in the predictions conducted for reflection on a single object with diffuse scattering, the LIM model is 26 times more efficient than ray-tracing.

- Hypothesis 4: LIM can consider the phenomena related to radio propagation such as transmission, reflection and diffuse scattering. In this Thesis, it has been shown that the LIM takes into account reflection and diffuse scattering easily since the materials used in the game engine already present these effects for light propagation. It has been also shown, in Chapter 5, that the material properties can be changed to present an interaction with the light similar to the interaction that those materials would present with radio waves in Millimeter Wave $(\mathrm{mmW})$ bands. Although transmission has not been considered in the tests conducted in this Thesis, the adaptation of the materials transparency is a possible 
way to emulate in the light domain the transmission characteristics that materials present in the radio waves domain.

- Hypothesis 5: Unity's game engine allows a complete simulation and visualization of $5 \mathrm{G}$ systems. In this Thesis, in Chapter 6 the use of Unity as a complete simulator for different use cases of $5 \mathrm{G}$ networks has been extensively demonstrated. In fact, it can be established that the simulation alternatives of the systems and the visualization platform based on Unity are unlimited and have great potential as a tool for applications in the industrial sector, security, vehicular communications, planning and dimensioning of mobile networks, among others. Unity, under the concept of "Serious Game Engineering", allows creating environments easily adaptable to the scientific community, education and all professionals involved in future $5 \mathrm{G}$ networks.

\subsection{Future Research Lines}

This Thesis is no more than the starting point for many other research topics. In particular, the following aspects can be highlighted:

- In order to complete LIM extensions, it is necessary to evaluate diffraction phenomena, also include human blocking and foliage attenuation. This extension will allow the use of LIM in dynamic scenarios with user mobility.

- It is necessary to extend the scope of LIM in order to make it valid in all types of scenarios. Specially for indoor cases, where the scenario's geometry is more complex and radio propagation is more challenging to characterize.

- As for LIM for indoor scenarios, characterization of typical indoor materials is necessary for a more accurate estimation. It is also necessary to expand research on the effect of roughness and thickness of materials, especially multi-layer materials which present different characteristics at different frequencies.

- The new use cases raised for $5 \mathrm{G}$ require visualization platforms where the user is allowed to interact dynamically and in real time with all the elements of the system. This Thesis has shown that it is feasible to use game engines for complete emulation of $5 \mathrm{G}$ systems. But there is still some work to be done, so it is necessary to go beyond the emulation of the radio channel to make this platform valid for complete $5 \mathrm{G}$ emulation. 
- Another point shown in this Thesis with great potential for future research is the use of game engines for emulation of vehicular communications in super realistic environments. In fact, in game engines it is possible to implement exact scenarios with the precise location of roads and traffic signs. It is also possible to integrate real traffic managers with Unity. And finally, it is possible to implement the components of the $5 \mathrm{G}$ networks along the roads. All these components will allow the development of a platform capable of emulating vehicle communications, and even evaluate the behavior of autonomous cars with algorithms of artificial intelligence. 



\section{Appendix A}

\section{Ray-Tracing Algorithm}

Deterministic models, in contrast to stochastic models, take into account the structure and geometry of the propagation environment. They reproduce all the geometric and electromagnetic parameters associated with a particular scenario. The most complex and accurate deterministic model of general use are ray-tracing models. This appendix describes the implementation of the raytracing model for the estimation of propagation losses.

\section{A.1 Properties of Dielectric Materials}

There is a dependence between the fading of signals and the dielectric properties of the materials with which radio waves interact. Indeed, an extensive study carried out in [73] define the properties of the main materials, both permittivity and conductivity, for a wide frequency range from 1 to $100 \mathrm{GHz}$. The calculation of the complex relative permittivity is given by

$$
\varepsilon^{*}=\varepsilon^{\prime}-j \varepsilon^{\prime \prime},
$$

where, the relative permittivity $\varepsilon^{\prime}$ for each material is

$$
\varepsilon^{\prime}=a \cdot f^{b}
$$

the complex permittivity $\varepsilon^{\prime \prime}$ is:

$$
\varepsilon^{\prime \prime}=17.98 \frac{\sigma}{f},
$$

the conductivity for each material is

$$
\sigma=c \cdot f^{d}
$$


where $f$ is the frequency in $\mathrm{GHz}, c$ the speed of light in vacuum and $d$ is the conductivity factor of the material. The material parameters are shown in Table A.1.

\begin{tabular}{|l|c|c|c|c|c|}
\hline \multirow{2}{*}{ Material } & \multicolumn{2}{|c|}{ Permittivity } & \multicolumn{2}{c|}{ Conductivity } & Frequency \\
\cline { 2 - 6 } & $\mathbf{a}$ & $\mathbf{b}$ & $\mathbf{c}$ & $\mathbf{d}$ & $\mathbf{G H z}$ \\
\hline Concrete & 5.31 & 0 & 0.0326 & 0.8095 & $1-100$ \\
\hline Metal & 1 & 0 & $10^{7}$ & 0 & $1-100$ \\
\hline Glass & 6.27 & 0 & 0.0043 & 1.0718 & $0.1-100$ \\
\hline Ground & 15 & -0.1 & 0.035 & 1.63 & $1-10$ \\
\hline Brick & 3.75 & 0 & 0.038 & 0.8095 & $1-100$ \\
\hline
\end{tabular}

Table A.1: Material electric parameters

\section{A.2 Free space propagation}

Free space losses are defined at the intensity that a signal decreases with respect to the distance it has traveled. Considering that the means of propagation is air, where you can approximate $\varepsilon=1$ and $\mu=1$ and assuming a unit gain and a lossless antenna, the basic attenuation is defined as:

$$
L_{f s}=\frac{P_{0}}{P_{R}}=\left(\frac{4 \pi r}{\lambda}\right)^{2},
$$

where $P_{0}$ is the power radiated by the transmitting antenna and $P_{R}$ is the power received by the receiving antenna.

The electric field in the far field domain is:

$$
E_{0}=\frac{\sqrt{30 P_{0} D_{G}(\theta, \phi)}}{r} e^{j k_{0} r}
$$

where $K_{0}=2 \pi / \lambda$ is the wave constant in free space, $D_{G}(\theta, \phi)$ is the antenna gain in azimuth and elevation and $r$ is the distance between transmitter and receiver.

\section{A.3 Specular Reflection}

Reflection is one of the dominant phenomena in radio propagation. A general solution to calculate the electric field of reflected rays lies in satisfying Maxwell's equations and contour conditions. Another point to consider, are the electromagnetic characteristics of the materials where the ray is reflected 


\section{A.4 Diffuse Scattering}

and the solution to this problem is found in the Fresnel coefficients. Indeed, the total electric field is divided into three components: a component of the incident beam, another component of the reflected beam and a portion that is transmitted through the material.

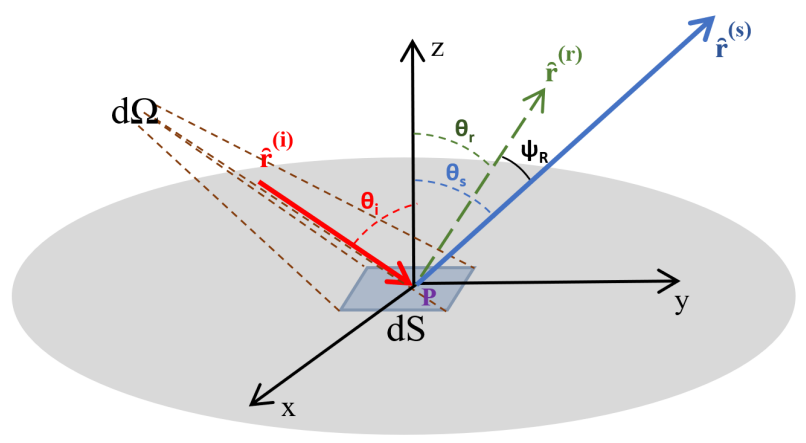

Figure A.1: Generic geometric model of specular reflection and diffuse scattering

The electric field of the reflected beam is given by:

$$
E=r_{x} \frac{e^{-\frac{j 2 \pi f d}{c}}}{d}
$$

where $r_{x}$ would be $r_{h}$ for the horizontal polarization and $r_{v}$ for the vertical polarization, and $d$ is the path length for the reflected ray (from the transmitter to the reflection point and from there to the receiver).

$$
r_{h}=\frac{\sin \psi-\sqrt{\varepsilon^{*}-\cos ^{2} \psi}}{\sin \psi+\sqrt{\varepsilon^{*}-\cos ^{2} \psi}}
$$

and

$$
r_{v}=\frac{\varepsilon^{*} \sin \psi-\sqrt{\varepsilon^{*}-\cos ^{2} \psi}}{\varepsilon^{*} \sin \psi+\sqrt{\varepsilon^{*}-\cos ^{2} \psi}}
$$

\section{A.4 Diffuse Scattering}

For diffuse scattering, the point $P$ is where diffuse scattering occurs (see Figure A.1), it has associated a coefficient of dispersion $S$ and a reflection coefficient $\Gamma$. In [113], two approach of $S$ are defined. The first, where all incident power is scattered and spreads in all directions. The second approach, where the 
scattering components also spreads in all directions but only considering the reflected energy. The first one, $S$ is defined by:

$$
S=\left.\frac{\left|\bar{E}_{S}\right|}{\left|\bar{E}_{i}\right|}\right|_{d S},
$$

where $E_{s}$ is the amplitude of the electric field of diffuse scattering, $E_{i}$ is the amplitude of the specular electric field and $P$ is the point where diffuse scattering is produced. The power balance due diffuse scattering, reflection and transmission in $P$ is defined as follows in [115]:

$$
1=\Gamma^{2} R^{2}+S^{2}+\frac{P_{p}}{P_{i}},
$$

where R (equation A.12) is the reflection loss factor, $0<R<1, \Gamma$ and $S$ are the scattering and reflection coefficient respectively, $P_{p}$ is the power that penetrate the material and $P_{i}$ is the incident power.

$$
R \cong \sqrt{1-\frac{S^{2}}{\Gamma^{2}}}
$$

In the second approach, $S$ is defined by equation A.13 and the power balance of equation A.14.

$$
\begin{gathered}
S=\left.\frac{\left|\bar{E}_{S}\right|}{\left|\bar{E}_{r}\right|}\right|_{d S} \\
1=\Gamma^{2}\left(R^{2}+S^{2}\right)+\frac{P_{p}}{P_{i}}
\end{gathered}
$$

With these two approaches, a function $U$ is defined as follows:

$$
U= \begin{cases}1 & \text { if } S=\left|\bar{E}_{S} / \bar{E}_{i}\right| \\ \Gamma & \text { if } S=\left|\bar{E}_{S} / \bar{E}_{R}\right| .\end{cases}
$$

In order to evaluate the measurements of the campaign, two diffuse scattering models are considered. The Lambertian model and the Directive model. The geometry shown in Figure A.1 is valid for both models.

\section{A.4.1 Lambertian Model}

In the Lambertian model, energy is scattered in all directions centered in the norm of the point $P$. In this model, the module of the diffuse electric field is defined by:

$$
\left|\bar{E}_{S}\right|^{2}=\left(\frac{K_{0} \cdot S}{r_{i} \cdot r_{s}}\right)^{2} \cdot U^{2} \cdot \frac{\cos \left(\theta_{i}\right) \cdot \cos \left(\theta_{s}\right)}{\pi} d S,
$$




\section{A.5 Diffraction}

where $r_{i}$ is the distance between the $T X$ and the point $P, r_{s}$ is the distance between RX and the point $P . \theta_{i}$ and $\theta_{s}$ are the incident and departure angles, and $K_{0}$,

$$
\left.K_{0}=\sqrt{60 G_{T X}(\beta) P_{t_{r a y}}}\right),
$$

where $G_{R X}(\beta)$ is the antenna gain in $\beta$ direction, and $P_{t_{\text {ray }}}$ is the ray power in W.

\section{A.4.2 Directive Model}

In the directive model, energy is scattered in the directions around reflection angles. It has two variants, directive and directive with back-scatter. The module of the electric field in the directive model is:

$$
\left|\bar{E}_{S}\right|^{2}=E_{S 0}^{2} \cdot\left(\frac{1+\cos \psi_{R}}{2}\right)^{\alpha_{R}},
$$

where $\psi_{R}$ is the angle between the scattering and specular rays, the exponent $\alpha_{R}$ is related to the width of the scattering lobe; the lobe will be wider when the exponent is greater. $E_{S 0}^{2}$ is defined by,

$$
E_{S 0}^{2}=\left(\frac{S K_{0}}{r_{i} r_{s}}\right)^{2} U^{2} \frac{d S \cos \left(\theta_{i}\right)}{F_{\alpha_{R}}},
$$

where $F_{\alpha_{R}}$ is,

$$
F_{\alpha_{R}}=\frac{1}{2^{\alpha_{R}}} \cdot \sum_{j=0}^{\alpha_{R}}\left(\begin{array}{c}
\alpha_{R} \\
j
\end{array}\right) \cdot I_{j}
$$

and $I_{j}$ is

$$
I_{j}=\frac{2 \pi}{j+1} \cdot\left[\cos \theta_{i} \sum_{w=0}^{\frac{j-1}{2}}\left(\begin{array}{c}
2 w \\
w
\end{array}\right) \cdot \frac{\sin ^{2 w} \theta_{i}}{2^{2 w}}\right]^{\frac{1-(-1)^{j}}{2}}
$$

\section{A.5 Diffraction}

Diffracted rays are produced by the incidence of rays that impact the edges or corners. The algorithms of Geometrical Optics (GO) and Uniform Theory of Diffraction (UTD) give good results for both indoor and outdoor environments. The algorithm of GO has a geometric connection between the incident ray and the diffracted rays. In the elevation view shown in the figure A.2, the incident ray reach the diffracting edge with an angle $\beta$ is the incident ray, and the 


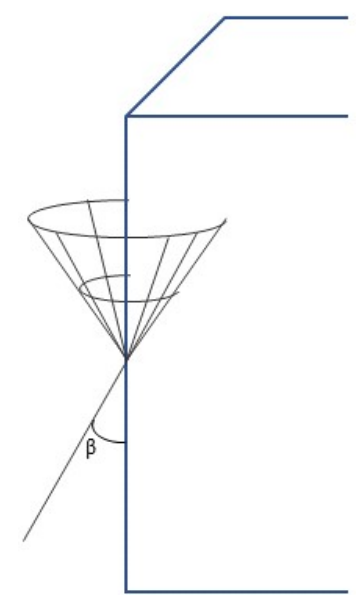

Figure A.2: Elevation view - Diffraction

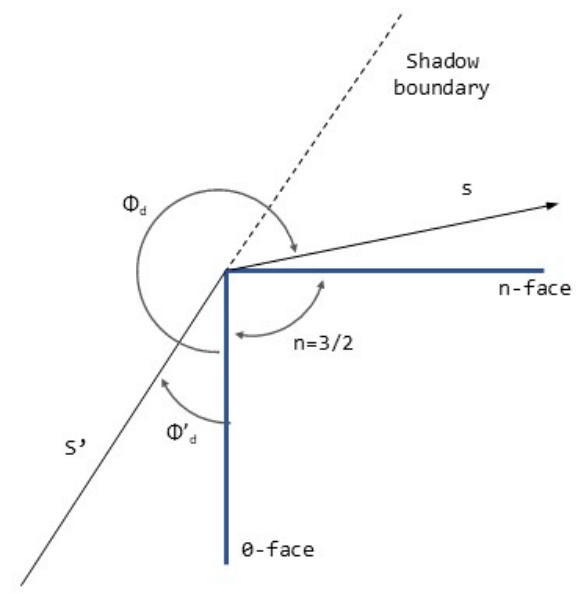

Figure A.3: Top view - Diffraction

diffracted moments come out in the form of a cone in all directions, known as Keller's cone [128].

In the top view, Figure A.3, the geometric properties of diffraction are described with the angles $\phi_{d}^{\prime}$ and $\phi_{d}$ for the outgoing beam, both with respect to face 0 . These geometrical properties must be known to calculate the diffraction coefficient, $D$. If both the transmitting and receiving points are known, the 


\section{A.5 Diffraction}

height of the intersection point can be calculated as:

$$
h=\frac{h_{T} d_{R}+h_{R} d_{T}}{d_{R}+d_{T}},
$$

where $h_{T}$ y $h_{R}$ are the transmitter and receiver heights respectively, $d_{T}$ is the distance between the diffraction point and the transmitter and $d_{R}$ is the distance between the diffraction point and the receiver.

The calculation of the diffraction coefficient is calculated as:

$$
D=\frac{-e^{-j \frac{\pi}{4}}}{2 n \sqrt{2 \pi k} \sin \beta} D_{1}+D_{2}+R_{0} D_{3}+R_{n} D_{4},
$$

where $k$ is a wave constant. $R_{0}$ and $R_{n}$ are the reflection coefficients on face 0 and face $\mathrm{n}$ respectively. The factor $n$ is calculated by knowing the angle $\alpha$ of the edge $n=2-\frac{\alpha}{\pi}$.

The components of diffraction are defined as:

$$
\begin{aligned}
& D_{1}=\cot \left[\frac{\pi+\left(\phi_{d}-\phi_{d}^{\prime}\right)}{2 n}\right] F\left(k L a^{+}\right), \\
& D_{2}=\cot \left[\frac{\pi-\left(\phi_{d}-\phi_{d}^{\prime}\right)}{2 n}\right] F\left(k L a^{-}\right), \\
& D_{3}=\cot \left[\frac{\pi-\left(\phi_{d}+\phi_{d}^{\prime}\right)}{2 n}\right] F\left(k L a^{-}\right), \\
& D_{4}=\cot \left[\frac{\pi+\left(\phi_{d}+\phi_{d}^{\prime}\right)}{2 n}\right] F\left(k L a^{+}\right),
\end{aligned}
$$

where $F(x)$ is the Fresnel transition function:

$$
F(x)=2 j \sqrt{x} e^{j x} \int_{\sqrt{x}}^{\infty} e^{-j \tau^{2}} d \tau .
$$

It is possible to simplify $F(x)$ both high values of $x$, with the following approximation:

$$
F(x) \simeq 1+j \frac{1}{2 x}-\frac{3}{4} \frac{1}{x^{2}}-j \frac{15}{8} \frac{1}{x^{3}}+\frac{75}{16} \frac{1}{x^{4}},
$$

and for small values of $x$

$$
F(x) \simeq\left[\sqrt{\pi x}-2 x e^{\frac{-j \pi}{4}}-\frac{2}{3} x^{2} e^{\frac{-j \pi}{4}}\right] e^{j\left(\frac{-j \pi}{4}+x\right)},
$$


where, the distance $L$ is given by:

$$
L=s \cdot \sin ^{2}(\beta)
$$

where $s$ is the distance between the receiver and the diffraction point. The function $a^{ \pm}$is defined as:

$$
a^{ \pm}\left(\phi_{d}-\phi_{d}^{\prime}\right)=2 \cos ^{2}\left[\frac{2 n \pi N^{ \pm}-\left(\phi_{d}-\phi_{d}^{\prime}\right)}{2}\right]
$$

where $N^{+}$and $N^{-}$are the nearest integers that satisfy the equations:

$$
2 n \pi N^{+}-\left(\phi_{d}-\phi_{d}^{\prime}\right)=\pi
$$

and

$$
2 n \pi N^{-}-\left(\phi_{d}-\phi_{d}^{\prime}\right)=\pi
$$

where $a^{+}, N^{+}$are associated with face $\mathrm{n}$ and $a^{-}, N^{-}$with the face 0 . Finally, the diffracted electric field it is given as:

$$
E_{d}=E_{0} D A e^{j k s} .
$$




\section{References}

[1] ITU-R, "IMT vision - framework and overall objectives of the future development of IMT for 2020 and beyond," ITU, Recommendation M.20830, Sep. 2015.

[2] M. Shafi, A. F. Molisch, P. J. Smith, T. Haustein, P. Zhu, P. De Silva, F. Tufvesson, A. Benjebbour, and G. Wunder, "5G: A tutorial overview of standards, trials, challenges, deployment, and practice," IEEE Journal on Selected Areas in Communications, vol. 35, no. 6, pp. 1201-1221, Jun. 2017.

[3] T. S. Rappaport, Y. Xing, G. R. MacCartney, A. F. Molisch, E. Mellios, and J. Zhang, "Overview of millimeter wave communications for fifthgeneration $(5 \mathrm{G})$ wireless networks - with a focus on propagation models," IEEE Transactions on Antennas and Propagation, vol. 65, no. 12, pp. 6213-6230, Aug. 2017.

[4] F. Boccardi, R. W. Heath, A. Lozano, T. L. Marzetta, and P. Popovski, "Five disruptive technology directions for 5G," IEEE Communications Magazine, vol. 52, no. 2, pp. 74-80, Feb. 2014.

[5] P. Marsch, Ö. Bulakci, O. Queseth, and M. Boldi, 5G System Design: Architectural and Functional Considerations and Long Term Research. Wiley, 2018.

[6] S. E. Elayoubi, M. Fallgren, P. Spapis, G. Zimmermann, D. MartínSacristán, C. Yang, S. Jeux, P. Agyapong, L. Campoy, Y. Qi et al., "5G service requirements and operational use cases: Analysis and METIS II vision," in 2016 European Conference on Networks and Communications (EuCNC), Sep. 2016, pp. 158-162.

[7] ITU-R, "Spectrum requirements for international mobile telecommunications 2000 (IMT-2000)," ITU, Recommendation M.2023, May 2000. 
[8] ITU-R, "Minimum requirements related to technical performance for IMT-2020 radio interface(s)," ITU, Recommendation M.2410-0, Nov. 2017.

[9] A. Osseiran, J. F. Monserrat, and P. Marsch, 5G mobile and wireless communications technology. Cambridge University Press, 2016.

[10] ITU-R, "Future technology trends of terrestrial IMT systems," ITU, Recommendation M.2320-0, Nov. 2014.

[11] S. M. Razavizadeh, M. Ahn, and I. Lee, "Three-dimensional beamforming: A new enabling technology for 5G wireless networks," IEEE Signal Processing Magazine, vol. 31, no. 6, pp. 94-101, Nov. 2014.

[12] E. Björnson, J. Hoydis, and L. Sanguinetti, "Massive MIMO has unlimited capacity," IEEE Transactions on Wireless Communications, vol. 17, no. 1, pp. 574-590, Jan. 2018.

[13] G. Fodor, N. Rajatheva, W. Zirwas, L. Thiele, M. Kurras, K. Guo, A. Tolli, J. H. Sorensen, and E. d. Carvalho, "An overview of massive MIMO technology components in METIS," IEEE Communications Magazine, vol. 55, no. 6, pp. 155-161, Jun. 2017.

[14] M. G. Sarret, G. Berardinelli, N. H. Mahmood, M. Fleischer, P. Mogensen, and H. Heinz, "Analyzing the potential of full duplex in 5G ultra-dense small cell networks," EURASIP Journal on Wireless Communications and Networking, vol. 2016, article no. 284, pp. 1-16, Dec. 2016.

[15] S. Gimenez, S. Roger, P. Baracca, D. Martín-Sacristán, J. Monserrat, V. Braun, and H. Halbauer, "Performance evaluation of analog beamforming with hardware impairments for $\mathrm{mmW}$ massive MIMO communication in an urban scenario," Sensors, vol. 16, no. 10, p. 1555, 2016.

[16] J. Lee, E. Tejedor, K. Ranta-aho, H. Wang, K. Lee, E. Semaan, E. Mohyeldin, J. Song, C. Bergljung, and S. Jung, "Spectrum for 5G: Global status, challenges, and enabling technologies," IEEE Communications Magazine, vol. 56, no. 3, pp. 12-18, Mar. 2018.

[17] S. Chen and J. Zhao, "The requirements, challenges, and technologies for 5G of terrestrial mobile telecommunication," IEEE Communications Magazine, vol. 52, no. 5, pp. 36-43, May 2014.

[18] E. Dahlman, S. Parkvall, and J. Skold, 5G NR: The next generation wireless access technology. Academic Press, Mar. 2018. 
[19] Y. Wang, J. Li, L. Huang, Y. Jing, A. Georgakopoulos, and P. Demestichas, "5G mobile: Spectrum broadening to higher-frequency bands to support high data rates," IEEE Vehicular Technology Magazine, vol. 9, no. 3, pp. 39-46, Sep. 2014.

[20] METIS, "METIS channel models," METIS, Deliverable D1.4, Feb. 2012.

[21] A. Kukushkin, Introduction to Mobile Network Engineering: GSM, 3GWCDMA, LTE and the Road to 5G. Wiley, Sep. 2018, ch. Mobile Radio Channel, pp. 27-47.

[22] T. Singal, Wireless communications. Tata McGraw-Hill Education, Mar. 2010.

[23] H. Zhao, R. Mayzus, S. Sun, M. Samimi, J. K. Schulz, Y. Azar, K. Wang, G. N. Wong, F. Gutierrez, and T. S. Rappaport, " $28 \mathrm{GHz}$ millimeter wave cellular communication measurements for reflection and penetration loss in and around buildings in New York city," in 2013 IEEE International Conference on Communications (ICC), Jun. 2013, pp. 5163-5167.

[24] T. S. Rappaport, S. Sun, R. Mayzus, H. Zhao, Y. Azar, K. Wang, G. N. Wong, J. K. Schulz, M. Samimi, and F. Gutierrez, "Millimeter wave mobile communications for $5 \mathrm{G}$ cellular: It will work!" IEEE access, vol. 1, pp. 335-349, May 2013.

[25] A. Kajiwara, "Line-of-sight indoor radio communication using circular polarized waves," IEEE Transactions on Vehicular Technology, vol. 44, no. 3, pp. 487-493, Aug. 1995.

[26] J. Pascual-García, J. Molina-García-Pardo, M. Martínez-Inglés, J. Rodríguez, and N. Saurín-Serrano, "On the importance of diffuse scattering model parameterization in indoor wireless channels at mm-Wave frequencies," IEEE Access, vol. 4, pp. 688-701, Feb. 2016.

[27] F. Sheikh, D. Lessy, M. Alissa, and T. Kaiser, "A comparison study of non-specular diffuse scattering models at terahertz frequencies," in 2018 First International Workshop on Mobile Terahertz Systems (IWMTS), Jul. 2018, pp. 1-6.

[28] J. Jürvelüinen and K. Haneda, "Sixty gigahertz indoor radio wave propagation prediction method based on full scattering model," Radio Science, vol. 49, no. 4, pp. 293-305, Apr. 2014. 
[29] C. Jansen, S. Priebe, C. Moller, M. Jacob, H. Dierke, M. Koch, and T. Kurner, "Diffuse scattering from rough surfaces in $\mathrm{THz}$ communication channels," IEEE Transactions on Terahertz Science and Technology, vol. 1, no. 2, pp. 462-472, Nov. 2011.

[30] R. Piesiewicz, C. Jansen, D. Mittleman, T. Kleine-Ostmann, M. Koch, and T. Kurner, "Scattering analysis for the modeling of $\mathrm{THz}$ communication systems," IEEE Transactions on Antennas and Propagation, vol. 55, no. 11, pp. 3002-3009, Nov. 2007.

[31] N. Blaunstein and J. B. Andersen, Multipath Phenomena in Cellular Networks. Artech House, Jun. 2002.

[32] M. M. Ahamed and S. Faruque, "Propagation factors affecting the performance of 5G millimeter wave radio channel," in 2016 IEEE International Conference on Electro Information Technology (EIT), May 2016, pp. $728-733$.

[33] G. R. MacCartney, Junhong Zhang, Shuai Nie, and T. S. Rappaport, "Path loss models for $5 \mathrm{G}$ millimeter wave propagation channels in urban microcells," in 2013 IEEE Global Communications Conference (GLOBECOM), Dec. 2013, pp. 3948-3953.

[34] C. Kourogiorgas, S. Sagkriotis, and A. D. Panagopoulos, "Coverage and outage capacity evaluation in $5 \mathrm{G}$ millimeter wave cellular systems: impact of rain attenuation," in 2015 9th European Conference on Antennas and Propagation (EuCAP), Apr. 2015, pp. 1-5.

[35] ITU-R, "Attenuation by atmospheric gases," ITU, Recommendation P.676-5, Feb. 2001.

[36] J. G. Andrews, S. Buzzi, W. Choi, S. V. Hanly, A. Lozano, A. C. Soong, and J. C. Zhang, "What will $5 \mathrm{G}$ be?" IEEE Journal on selected areas in communications, vol. 32, no. 6, pp. 1065-1082, Jun. 2014.

[37] 3GPP TSG RAN, "Study on channel model for frequencies from 0.5 to $100 \mathrm{GHz}, " 3 \mathrm{GPP}, \mathrm{TR} 38.901$ V14.0.0, May 2017.

[38] M. K. Samimi, T. S. Rappaport, and G. R. MacCartney, "Probabilistic omnidirectional path loss models for millimeter-wave outdoor communications," IEEE Wireless Communications Letters, vol. 4, no. 4, pp. 357-360, Aug. 2015.

[39] ITU-R, "Guidelines for evaluation of radio interface technologies for IMT2020," ITU, Recommendation M.2412-0, Oct. 2017. 
[40] Shu Sun, Theodore S. Rappaport, "Channel modeling and multi-cell hybrid beamforming for fifth-generation millimeterwave wireless communications," NYU, TR 2018-001, May 2018.

[41] J. Järveläinen, S. L. H. Nguyen, K. Haneda, R. Naderpour, and U. T. Virk, "Evaluation of millimeter-wave line-of-sight probability with point cloud data," IEEE Wireless Communications Letters, vol. 5, no. 3, pp. 228-231, Jun. 2016.

[42] G. Liang and H. L. Bertoni, "A new approach to 3-D ray tracing for propagation prediction in cities," IEEE Transactions on Antennas and Propagation, vol. 46, no. 6, pp. 853-863, Jun. 1998.

[43] M. K. Samimi and T. S. Rappaport, "3-D millimeter-wave statistical channel model for 5G wireless system design," IEEE Transactions on Microwave Theory and Techniques, vol. 64, no. 7, pp. 2207-2225, Jul. 2016.

[44] E. J. Violette, R. H. Espeland, R. O. DeBolt, and F. K. Schwering, "Millimeter-wave propagation at street level in an urban environment," IEEE Transactions on Geoscience and Remote Sensing, vol. 26, no. 3, pp. 368-380, May 1988.

[45] D. M. Matic, H. Harada, and R. Prasad, "Indoor and outdoor frequency measurements for mm-waves in the range of $60 \mathrm{GHz}$," in 48th IEEE Vehicular Technology Conference (VTC), May 1998, pp. 567-571.

[46] P. F. Smulders and L. Correia, "Characterisation of propagation in 60 GHz radio channels," Electronics and Communication Engineering Journal, vol. 9, no. 2, pp. 73-80, Apr. 1997.

[47] E. Ben-Dor, T. S. Rappaport, Y. Qiao, and S. J. Lauffenburger, "Millimeter-wave $60 \mathrm{GHz}$ outdoor and vehicle AoA propagation measurements using a broadband channel sounder," in 2011 IEEE Global Telecommunications Conference (GLOBECOM), Jan. 2011, pp. 1-6.

[48] J. S. Lu, P. Cabrol, D. Steinbach, and R. V. Pragada, "Measurement and characterization of various outdoor $60 \mathrm{GHz}$ diffracted and scattered paths," in 2013 IEEE Military Communications Conference (MILCOM), Feb. 2013, pp. 1238-1243.

[49] G. R. Maccartney, T. S. Rappaport, M. K. Samimi, and S. Sun, "Millimeter-wave omnidirectional path loss data for small cell $5 \mathrm{G}$ channel modeling," IEEE Access, vol. 3, pp. 1573-1580, Aug. 2015. 
[50] Y. Azar, G. N. Wong, K. Wang, R. Mayzus, J. K. Schulz, H. Zhao, F. Gutierrez, D. Hwang, and T. S. Rappaport, " $28 \mathrm{GHz}$ propagation measurements for outdoor cellular communications using steerable beam antennas in New York city," in 2013 IEEE International Conference on Communications (ICC), Jun. 2013, pp. 5143-5147.

[51] G. R. Maccartney, T. S. Rappaport, S. Sun, and S. Deng, "Indoor office wideband millimeter-wave propagation measurements and channel models at 28 and $73 \mathrm{GHz}$ for ultra-dense 5G wireless networks," IEEE Access, vol. 3, pp. 2388-2424, Dec. 2015.

[52] S. Nie, G. R. MacCartney, S. Sun, and T. S. Rappaport, "72 GHz millimeter wave indoor measurements for wireless and backhaul communications," in 2013 IEEE 24th Annual International Symposium on Personal, Indoor, and Mobile Radio Communications (PIMRC), Sep. 2013, pp. 2429-2433.

[53] S. Deng, M. K. Samimi, and T. S. Rappaport, "28 GHz and $73 \mathrm{GHz}$ millimeter-wave indoor propagation measurements and path loss models," in 2015 IEEE International Conference on Communication Workshop (ICCW), Jun. 2015, pp. 1244-1250.

[54] R. Dörner, S. Göbel, W. Effelsberg, and J. Wiemeyer, Serious Games. Springer, Jan. 2016.

[55] T. Susi, M. Johannesson, and P. Backlund, "Serious games: An overview," School of Humanities and Informatics, Nov. 2005.

[56] D. Learning, "The digital game-based learning revolution," Learning, vol. 1, no. 1, pp. 1-19, May 2001.

[57] D. Djaouti, J. Alvarez, and J.-P. Jessel, "Classifying serious games: the G/P/S model," in Handbook of research on improving learning and motivation through educational games: Multidisciplinary approaches. IGI Global, Jan. 2011, pp. 118-136.

[58] Unity, "Unity 3d," 2019. [Online]. Available: URL\{https://unity.com\}

[59] M. Oliveira, N. Pereira, E. Oliveira, J. E. Almeida, and R. Rossetti, "A multi-player approach in serious games: testing pedestrian fire evacuation scenarios," in Doctoral Symposium in Informatics Engineering (DSIE), Jan. 2015. 
[60] J. Ribeiro, J. E. Almeida, R. J. Rossetti, A. Coelho, and A. L. Coelho, "Towards a serious games evacuation simulator," arXiv preprint arXiv:1303.3827, Jun. 2013.

[61] J. Ribeiro, J. E. Almeida, R. J. Rossetti, A. Coelho, and A. L. Coelho, "Using serious games to train evacuation behaviour," in 7th Iberian Conference on Information Systems and Technologies (CISTI 2012), Jun. 2012, pp. 1-6.

[62] Y. Xu, E. Kim, K. Lee, J. Ki, and B. Lee, "FDS simulation high rise building model for unity 3D game engine," International Journal of Smart Home, vol. 7, no. 5, pp. 263-274, Jul. 2013.

[63] A. Navarro, J. V. Pradilla, and O. Rios, "Open source 3D game engines for serious games modeling," Modeling and Simulation in Engineering, pp. 143-158, Mar. 2012.

[64] A. Navarro, D. Guevara, and N. Cardona, "Delay spread estimation in 4,2 $\mathrm{GHz}$ band using game ray based method," in 2016 IEEE International Symposium on Antennas and Propagation (APSURSI), Jun. 2016, pp. 1453-1454.

[65] A. Navarro and D. Guevara, "Applicability of game engine for ray tracing techniques in a complex urban environment," in 72nd IEEE Vehicular Technology Conference (VTC Fall), Sep. 2010, pp. 1-5.

[66] A. Navarro, D. Guevara, and S. Londoño, "Using 3-D video game technology in channel modeling," IEEE Access, vol. 2, pp. 1652-1659, Nov. 2014.

[67] A. Navarro, D. Guevara, and N. Cardona, "Game engines ray-tracing models for indoor channel modeling," in 2014 IEEE Antennas and Propagation Society International Symposium (APSURSI), Jul. 2014, pp. 1163-1164.

[68] E. Egea-Lopez, F. Losilla, J. Pascual-Garcia, and J. M. Molina-GarciaPardo, "Vehicular networks simulation with realistic physics," IEEE Access, vol. 7, pp. 44021-44036, Apr. 2019.

[69] C. Herranz, D. Martín-Sacristán, J. F. Monserrat, N. Cardona, and S. Inca, "On the use of serious game engineering for 5G system performance evaluation," in European Conference on Networks and Communications 2016, Jun. 2016. 
[70] D. Martín-Sacristán, C. Herranz, J. F. Monserrat, A. Szczygiel, N. P. Kuruvatti, D. Garcia-Roger, D. Prado, M. Boldi, J. Belschner, and H. D. Schotten, "5G visualization: The METIS-II project approach," Mobile Information Systems, vol. 2018, Sep. 2018.

[71] J. F. Monserrat, S. Inca, J. Calabuig, and D. Martín-Sacristán, "Mapbased channel model for urban macrocell propagation scenarios," International journal of Antennas and Propagation, vol. 2015, Apr. 2015.

[72] A. Osseiran, F. Boccardi, V. Braun, K. Kusume, P. Marsch, M. Maternia, O. Queseth, M. Schellmann, H. Schotten, H. Taoka et al., "Scenarios for $5 \mathrm{G}$ mobile and wireless communications: the vision of the METIS project," IEEE communications magazine, vol. 52, no. 5, pp. 26-35, 2014.

[73] ITU-R, "Effects of building materials and structures on radiowave propagation above about $100 \mathrm{MHz}$," ITU, Recommendation P.2040-1, Jul. 2015.

[74] B. Mondal, T. A. Thomas, E. Visotsky, F. W. Vook, A. Ghosh, Y.-H. Nam, Y. Li, J. Zhang, M. Zhang, Q. Luo et al., "3D channel model in 3GPP," IEEE Communications Magazine, vol. 53, no. 3, pp. 16-23, Mar. 2015.

[75] 3GPP TSG RAN, "Study on channel model for frequency spectrum above $6 \mathrm{GHz}, " 3 \mathrm{GPP}, \mathrm{TR} 38.900$ V14.2.0, Jun. 2017.

[76] J. Monserrat, R. Fraile, and L. Rubio, "Application of alternating projection method to ensure feasibility of shadowing cross-correlation models," Electronics Letters, vol. 43, no. 13, pp. 724-725, 2007.

[77] S. Sun, T. S. Rappaport, T. A. Thomas, A. Ghosh, H. C. Nguyen, I. Z. Kovács, I. Rodriguez, O. Koymen, and A. Partyka, "Investigation of prediction accuracy, sensitivity, and parameter stability of large-scale propagation path loss models for 5G wireless communications," IEEE Transactions on Vehicular Technology, vol. 65, no. 5, pp. 2843-2860, 2016.

[78] T. S. Rappaport, Y. Xing, G. R. MacCartney, A. F. Molisch, E. Mellios, and J. Zhang, "Overview of millimeter wave communications for fifthgeneration (5G) wireless networks - with a focus on propagation models," IEEE Transactions on Antennas and Propagation, vol. 65, no. 12, pp. 6213-6230, Dec. 2017.

[79] 5GCM, "5G channel model for bands up to $100 \mathrm{GHz}$," 5GCM, TR 201610, Oct. 2016. 
[80] L. Rubio, J. Monserrat, A. P. Garcia, and J. Reig, "Temporal dispersion characterization of over-building propagation applied to iso-frequency repeaters," Antennas and Wireless Propagation Letters, IEEE, vol. 8, pp. 509-512, Feb. 2009.

[81] ETSI, "Universal mobile telecommunications system (UMTS); selection procedures for the choice of radio transmission technologies of the UMTS," ETSI, TR 101112 v3.1.0, Nov. 1997.

[82] ITU-R, "Propagation data and prediction methods for the planning of indoor radiocommunication systems and radio local area networks in the frequency range $300 \mathrm{MHz}$ to $100 \mathrm{GHz}$," ITU, Recommendation P.1238-9, Jun. 2017.

[83] G. R. MacCartney and T. S. Rappaport, "73 GHz millimeter wave propagation measurements for outdoor urban mobile and backhaul communications in New York City," in 2014 IEEE International Conference on Communications (ICC), Aug. 2014, pp. 4862-4867.

[84] T. S. Rappaport, G. R. MacCartney, M. K. Samimi, and S. Sun, "Wideband millimeter-wave propagation measurements and channel models for future wireless communication system design," IEEE Transactions on Communications, vol. 63, no. 9, pp. 3029-3056, May 2015.

[85] Unity, "Installing the Unity specific version," 2019. [Online]. Available: URL\{https://unity3d.com/get-unity/download/archive\}

[86] Unity, "Unity asset store," 2019. [Online]. Available: URL\{https: //assetstore.unity.com/\}

[87] Unity, "City pack," 2019. [Online]. Available: URL\{https://assetstore. unity.com/packages/3d/props/exterior/city-pack- 42685$\}$

[88] Unity, "Hazelwood loft," 2019. [Online]. Available: URL\{https: //www.artstation.com/artwork/meQye\}

[89] Unity, "Modular traffic for playmaker," 2019. [Online]. Available: URL\{https://assetstore.unity.com/packages/templates/packs/ modular-traffic-for-playmaker-40764\}

[90] Unity, "Intelligent traffic system (source)," 2019. [Online]. Available: URL\{https://assetstore.unity.com/packages/templates/systems/ its-intelligent-traffic-system-source-22765\} 
[91] Unity, "Artificial neural networks," 2019. [Online]. Available: URL\{https://https://assetstore.unity.com/packages/tools/ai/ artificial-neural-networks-93236\}

[92] Unity, "Apex utility ai - personal edition," 2019. [Online]. Available: URL\{https://assetstore.unity.com/packages/tools/ visual-scripting/apex-utility-ai-personal-edition-56306\}

[93] W. Goldstone, Unity game development essentials. Packt Publishing Ltd, 2009.

[94] Unity, "Unity global illumination," 2019. [Online]. Available: URL\{https: //docs.unity3d.com/Manual/GIIntro.html\}

[95] P. Dutre, P. Bekaert, and K. Bala, Advanced global illumination. AK Peters/CRC Press, Jun. 2006.

[96] J. Arvo et al., "Backward ray tracing," in Developments in Ray Tracing, Computer Graphics, Proc. of ACM SIGGRAPH 86 Course Notes, Sep. 1986, pp. 259-263.

[97] S. Teller and P. Hanrahan, "Global visibility algorithms for illumination computations," in 20th annual conference on Computer graphics and interactive techniques, Mar. 1993, pp. 239-246.

[98] A. Navarro, J. V. Pradilla, and P. Madriñán, "A 3D game tool for mobile networks planning," in 2010 Second International Conference on Mobile, Hybrid, and On-Line Learning, Feb. 2010, pp. 158-161.

[99] METIS-II, "Final 5G visualization," METIS-II, Deliverable D7.3, Jun. 2017.

[100] P. Chakraborty, N. P. Kuruvatti, and H. D. Schotten, "A novel serious game engineering based interactive visualization and evaluation platform for cellular technologies," in 2017 International Symposium on Networks, Computers and Communications (ISNCC), Sep. 2017, pp. 1-6.

[101] M. Haklay and P. Weber, "Openstreetmap: User-generated street maps," IEEE Pervasive Computing, vol. 7, no. 4, pp. 12-18, Oct. 2008.

[102] F. Husain, S. M. Hussein, T. A. Mastor, A. R. A. Rasam, A. M. Samad, I. Ma'arof, and K. A. Hashim, "A review of web-based GIS for visualization of George Town, Penang sustainability as world heritage site," in 2013 IEEE International Conference on Control System, Computing and Engineering, Nov. 2013, pp. 435-440. 
[103] D. Wilkie, J. Sewall, and M. C. Lin, "Transforming GIS data into functional road models for large-scale traffic simulation," IEEE Transactions on Visualization and Computer Graphics, vol. 18, no. 6, pp. 890-901, Jun. 2011.

[104] S. Wang, Z. Mao, C. Zeng, H. Gong, S. Li, and B. Chen, "A new method of virtual reality based on Unity3D," in 18th International Conference on Geoinformatics, Sep. 2010, pp. 1-5.

[105] Y. Zhao, C. Yan, X. Zhou, Q. Zhu, S. Wang, and W. Guo, "The research and development of 3D urban geographic information system with Unity3D," in 21st International Conference on Geoinformatics, Jun. 2013, pp. 1-4.

[106] M. Kyro, V.-M. Kolmonen, and P. Vainikainen, "Experimental propagation channel characterization of mm-wave radio links in urban scenarios," IEEE Antennas and Wireless Propagation Letters, vol. 11, pp. 865-868, 2012.

[107] V. Degli-Esposti, F. Fuschini, E. M. Vitucci, and G. Falciasecca, "Measurement and modelling of scattering from buildings," IEEE Transactions on Antennas and Propagation, vol. 55, no. 1, pp. 143-153, Jan. 2007.

[108] V. Degli-Esposti, D. Guiducci, A. de'Marsi, P. Azzi, and F. Fuschini, "An advanced field prediction model including diffuse scattering," IEEE Transactions on Antennas and Propagation, vol. 52, no. 7, pp. 1717-1728, Jul. 2004.

[109] F. Fuschini, V. Degli-Esposti, and E. M. Vitucci, "A model for forwarddiffuse scattering through a wall," in 4th European Conference on Antennas and Propagation (EuCAP), Apr. 2010, pp. 1-4.

[110] L. Minghini, R. D’Errico, V. Degli Esposti, and E. M. Vitucci, "Electromagnetic simulation and measurement of diffuse scattering from building walls," in 8th European Conference on Antennas and Propagation (EuCAP), Apr. 2014, pp. 1298-1302.

[111] J. Pascual-Garcia, M. Martinez-Ingles, J. Molina-Garcia-Pardo, J. Rodríguez, and V. Degli-Esposti, "Experimental parameterization of a diffuse scattering model at $60 \mathrm{GHz}$," in 2015 IEEE-APS Topical Conference on Antennas and Propagation in Wireless Communications (APWC), Sep. 2015, pp. 734-737. 
[112] F. Mani, E. M. Vitucci, M. Barbiroli, F. Fuschini, V. degli Esposti, M. Gan, C. Li, J. Zhao, and Z. Zhong, " $26 \mathrm{GHz}$ ray-tracing pathloss prediction in outdoor scenario in presence of vegetation," in 12th European Conference on Antennas and Propagation (EuCAP), Apr. 2018, pp. $1-5$.

[113] V. Degli-Esposti, "A diffuse scattering model for urban propagation prediction," IEEE Transactions on Antennas and Propagation, vol. 49, no. 7, pp. 1111-1113, Jul. 2001.

[114] V. Degli-Esposti and H. L. Bertoni, "Evaluation of the role of diffuse scattering in urban microcellular propagation," in 50th IEEE Vehicular Technology Conference (VTC Fall), Sep. 1999, pp. 1392-1396.

[115] V. Degli-Esposti, F. Fuschini, E. M. Vitucci, and G. Falciasecca, "Measurement and modelling of scattering from buildings," IEEE Transactions on Antennas and Propagation, vol. 55, no. 1, pp. 143-153, Jan. 2007.

[116] M. Oren and S. K. Nayar, "Generalization of Lambert's reflectance model," in Proceedings of the 21st Annual Conference on Computer Graphics and Interactive Techniques, Jul. 1994, pp. 239-246.

[117] D. López-Pérez, M. Ding, H. Claussen, and A. H. Jafari, "Towards 1 gbps/ue in cellular systems: Understanding ultra-dense small cell deployments," IEEE Communications Surveys Tutorials, vol. 17, no. 4, pp. 2078-2101, sep 2015.

[118] WINNER II, "WINNER II channel models," IST-4-027756 WINNER II, Deliverable D1.1.2 V1.2, Feb. 2008.

[119] O. Ozgun, "New software tool (GO+UTD) for visualization of wave propagation [testing ourselves]," IEEE Antennas and Propagation Magazine, vol. 58, no. 3, pp. 91-103, Jun. 2016.

[120] METIS-II, "Preliminary 5G visualization," METIS-II, Deliverable D7.2, Dec. 2016.

[121] METIS, "METIS Simulation Resources," 2015. [Online]. Available: URL\{https://metis2020.com/documents/simulations/index.html\}

[122] MatSim, "MATSim Documentation," 2019. [Online]. Available: URL\{https://matsim.org/docs/\} 


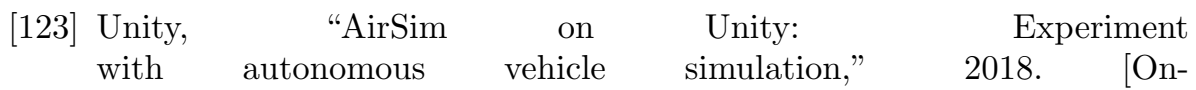
line]. Available: URL\{https://blogs.unity3d.com/es/2018/11/14/ airsim-on-unity-experiment-with-autonomous-vehicle-simulation/\}

[124] Unity, "IBM Watson SDK for Unity," 2018. [Online]. Available: URL\{https://assetstore.unity.com/packages/tools/ai/ ibm-watson-sdk-for-unity-108831\}

[125] H. Jung, "Cisco visual networking index: global mobile data traffic forecast update 2010-2015," Cisco Systems, Deliverable, Feb. 2011.

[126] D. Prado, S. Inca, D. Martín-Sacristán, and J. F. Monserrat, "Comparison of optimization methods for aerial base station placement with users mobility," in 2019 European Conference on Networks and Communications (EuCNC), jun 2019, pp. 485-489.

[127] M. Erdelj, E. Natalizio, K. R. Chowdhury, and I. F. Akyildiz, "Help from the sky: leveraging UAVs for disaster management," IEEE Pervasive Computing, vol. 16, no. 1, pp. 24-32, Jan. 2017.

[128] Y. Rahmat-Samii, "GTD, UTD, UAT and STD: A historical revisit," 2012 IEEE-APS Topical Conference on Antennas and Propagation in Wireless Communications (APWC), pp. 1145-1148, Oct. 2012. 
